\title{
ENHANCED DETECTION OF CORROSION AND COATING AGING MECHANISMS USING
} CORE-SHELL QUANTUM DOTS

\author{
A Thesis \\ presented to \\ the Faculty of California Polytechnic State University, \\ San Luis Obispo
}

\author{
In Partial Fulfillment \\ of the Requirements for the Degree \\ Master of Polymers and Coatings Science
}

by

Raymond Bao Thai

June 2018 
(C) 2018

Raymond Bao Thai

ALL RIGHTS RESERVED 


\section{COMMITTEE MEMBERSHIP}

TITLE: Enhanced Detection of Corrosion and Coating Aging Mechanisms Using Core-Shell Quantum Dots

AUTHOR: Raymond Bao Thai

DATE SUBMITTED: June 2018

COMMITTEE CHAIR: Erik D. Sapper, Ph.D. Assistant Professor of Chemistry

COMMITTEE MEMBER: Ivan Cole, Ph.D. ECP Director

Royal Melbourne Institute of Technology

COMMITTEE MEMBER: Adrian Trinchi, Ph.D.

Senior Scientist

Commonwealth Scientific and Industrial Research Organization

COMMITTEE MEMBER: Ray Fernando, Ph.D. Professor of Chemistry Endowed Chair for Polymers \& Coatings 


\begin{abstract}
Enhanced Detection of Corrosion and Coating Aging Mechanisms Using Core-Shell Quantum Dots

Raymond Bao Thai
\end{abstract}

Understanding aging mechanisms in coatings during their serviceable life is complicated, due to the inherent stochastic nature of exposure profiles, service environments, and rare weather events. Thus, the development of improved test methods for service life prediction and accelerated weathering relies on the availability of infallible degradation and exposure data, delivered as a function of cumulative time in service. In a related application, non-destructive evaluation methods rely on the premise of being able to probe or monitor in situ mechanisms within a coating system without interfering with actual aging mechanisms. Ultimately, quantum dots can be utilized as in situ sensors for the detection, ongoing monitoring, and quantification of environmental exposure and degradation of coating systems exposed to humidity, moisture, electrolyte, and ultraviolet radiation cycles. Synthetic pathways for producing formulation-ready quantum dots are presented, along with preliminary results from controlled exposure and degradation studies. Current and ongoing work involves extending this technology to industrial nondestructive evaluation and coating health monitoring applications. 


\section{ACKNOWLEDGMENTS}

There are several people that have been instrumental in proving guidance and aid required for me to complete this project. Thank you for helping me with any avenue of this project and motivating me towards becoming the best possible scientist I can be. My experience stateside at Cal Poly and abroad at CSIRO has shaped me and encouraged my growth - both personally and professionally - in ways that I could never have imagined.

Thank you, Dr. Erik Sapper, for recognizing an opportunity to study and live abroad in Melbourne and selecting me of all people to undergo the endeavor. Your relationship with Dr. Ivan Cole is a constant reminder that friendships do not need proximity to flourish. Despite Dr. Cole constantly asking me if you respond to me (since you don't respond to him), he respects you greatly, and the bond you two share was crucial for sending me over to do research at his lab. Thank you to Dr. Cole as well for being patient with my work and encouraging me every step of the way to keep pushing. A special thank you to Dr. Ivan Cole for arranging RMIT and my travel visa.

To all my CSIRO family, thank you for making the workplace environment a memory that I will never forget. In particular, I would like to thank Dr. Adrian Trinchi for taking me under his wing and showing me the ropes with the inorganic synthesis of quantum dot systems. Thank you to Dr. Linda Varadi for being the resident expert in organic synthesis and allowing me to ask questions such as how to use a Schlenk line or where to dispose of the many organic solvents I used to keep my glassware clean. Also thank you to Dr. Michael Breedon and Dr. Hong Yin for helping me with general lab equipment and logistical support.

Thank you to Dr. Ray Fernando and the Kenneth N. Edwards Western Coatings Technology Center for sponsoring and supporting the work. Thank you for the funding by the Bill Moore Fellowships, and a special thank you to Ms. Candace D. McNabb for ensuring that the funds get into my bank account ASAP!

Thank you to my favorite mentor Dr. Jennifer Hoffman, who kept in touch with me constantly despite being back home in the Bay Area. You continue to surprise me with your timely text messages and emails, and often times, I would receive them out of the blue and they would be just what I needed to get the right attitude for the day. I am so happy to be back home in the Bay Area so we can reconnect. Thank you for always supporting me and reminding me that my dreams can be achieved!

I really cannot stress enough how fortunate I am to have been able to take some time away from home and have it count for my master's thesis work at Cal Poly. It truly has been an opportunity of a lifetime, and there have been so many special memories because of it. 


\section{TABLE OF CONTENTS}

List of Tables

List of Figures

\section{CHAPTER}

1 Quantum Dots as Nanomaterials Used in Coatings .................................. 1

2 Quantum Dot Basics ............................................................ 9

2.1 Band Gap Energy and Density of States ...................................... 10

2.2 Physics Behind Core-Shell Quantum Dots .................................... 16

2.3 Quantum Dots as Sensing Materials ............................................ 19

2.4 Fluorescence Quenching to Detect Heavy Metal Ions ............................. 22

2.5 Transition for Use in Sensing for Corrosion Events ............................ 28

3 Review of Corrosion and Its Redox Reactions .................................... 30

3.1 Performance Additives in Organic Coatings Systems .............................. 31

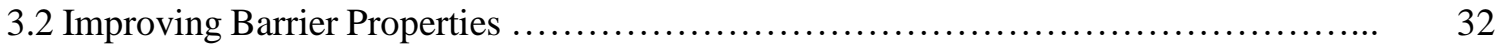

3.3 Surface Preparation for Better Coating Deposition .............................. 33

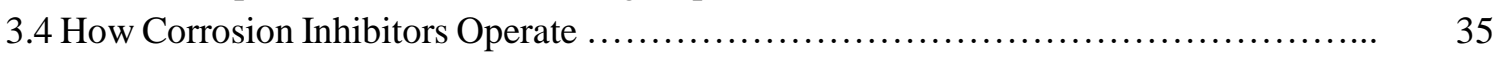

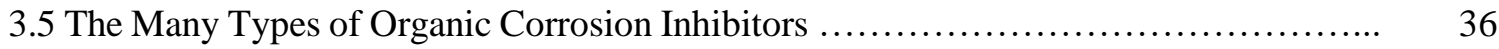

3.6 Surface Modification for Increased Quantum Dot Passivity ....................... 41

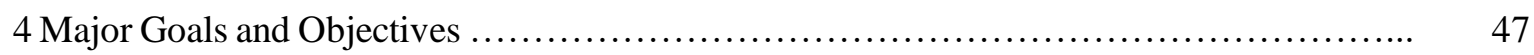

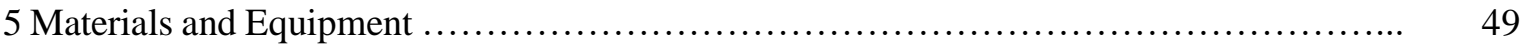

6 Core Synthesis - Making the Cadmium Selenide Cores ............................ 51

6.1 Centrifugation and Purification of the CdSe Cores ................................ 58

6.2 Optical Characterization Results for the CdSe Cores ............................. 65

6.3 Calculating the Amounts of $\mathrm{Zn}$ and $\mathrm{S}$ Injection Precursors .......................... 66

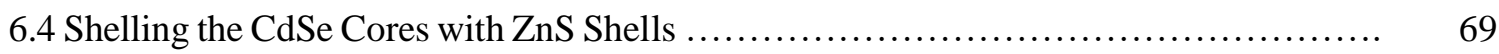

7 Core-Shell Synthesis - Making the CdSe/ZnS Core-Shell Quantum Dots ................. 72

7.1 Centrifugation and Purification of CdSe/ZnS Core-Shell Quantum Dots .............. 77

7.2 Optical Characterization Results for the CdSe/ZnS Core-Shell Quantum Dots ........... 80

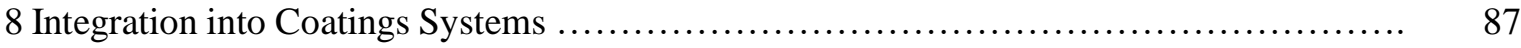

8.1 Deposition of Quantum Dots into Coatings .................................. 90

8.2 Preliminary Immersion Aging Testing ...................................... 92

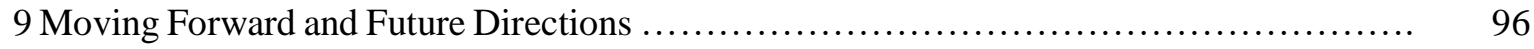

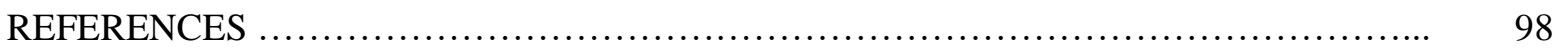

APPENDICES 104

A. Synthesis Procedure for CdSe/ZnS Quantum Dots ............................. 104 


\section{LIST OF TABLES}

Table

Page

5.1. Molar Masses and Densities of Synthesis Chemicals

6.1. Example synthesis measurements for preparing the cadmium source

6.2. Example synthesis measurements for preparing the selenium source

6.3. Example synthesis measurements for preparing the cadmium source, scaled up $3 \mathrm{x} \ldots \ldots$.

6.4. Example synthesis measurements for preparing the selenium source, scaled up $3 \mathrm{x} \ldots \ldots$

6.5. Determining the Change in Volume Between Inner and Outer Spheres

6.6. Converting $\Delta \mathrm{V}$ to Mass to Determine Shelling Injection Volumes

68

7.1. Example Setup for Shelling the CdSe Cores - Abs 0.383 at $558 \mathrm{~nm}$

7.2. Example Shelling Amounts and Timetables

8.1. Summary of Polymers and Coatings Systems Used

8.2. Deposition into Polymers

8.3. Deposition into Commercial and Specialty Paint Systems

8.4. Post-Immersion for Polymers 


\section{LIST OF FIGURES}

Figure Page

1.1 Melting point depression occurs approaching the nanoscale due to increased surface energy at smaller dimensions for a variety of metals

1.2 The glass transition temperature decreases for polystyrene as it approaches the nanoscale

1.3. The glass transition temperature decreases with the HMDS treatment due to intermolecular forces interacting with the poly(methyl methacrylate) as it approaches the nanoscale

1.4. The glass transition temperature decreases for free-standing polystyrene thin films at the nanoscale

1.5. The glass transition temperature increases for polystyrene thin films supported by hydrogen bonding on a silicon wafer at the nanoscale

1.6. Nanoclays must be surface treated to transition from the intercalated to exfoliated state to maximize dispersion and performance increase in the nanocomposite

1.7. Examples of nanoparticles and their properties that can be embedded into coatings systems

2.1. Surface area to volume ratios increase as materials become smaller; here (a) has a surface area to volume ratio of $6: 1$ while (b) is $3: 1$ and (c) is $2: 1$

2.2. An electron-hole pair is generated when enough energy is provided into the system to excite an electron from the valence band to the conduction band - different materials require different energy as the band gap

2.3. Confinement refers to restricting the possible degrees of freedom in which the electron-hole pair can be located - as particle size decreases, dimensionality decreases and exists as a discrete quantized energy level; dimensionality is observed as (a) 0-D, (b) 1-D, (c) 2-D, and (d) 3-D

2.4. As quantum dot nanoparticles become larger, the color fluoresced changes, represented as larger and larger wavelengths in the visible light spectrum

2.5. Green color $-525 \mathrm{~nm}$

2.6. Light yellow color $-575 \mathrm{~nm}$

2.7. Orange color $-610 \mathrm{~nm}$

2.8. Light red color $-650 \mathrm{~nm}$

2.9. Schematic of a core-shell quantum dot with attached capping molecules

2.10. Various types of configurations for systems where there are multiple band gaps core-shell quantum dots generally focus on Type I systems to optimize quantum yield and performance

2.11. Example of quantum dot for sensing application: (a) schematic of glucose oxidase reacting with glucose, (b) fluorescence quenching as concentration of glucose increases, (c) percent quenched for standard glucose solution added 
2.12. Quantum dot sensing application where the attached ring structure is $\mathrm{pH}$ sensitive (a) fluorescence quenching as fluoride concentration is increased, (b) plot of relative intensity of fluorescence against log [fluoride], (c) image of "on" and "off" states, and (d) plot of relative intensity against concentration for 5 titrated against fluoride stock solution

2.13. The presence of an appropriate analyte interacts with the capping agent on the surface of the quantum dot system, thereby disrupting the characteristic fluorescence and decreasing its intensity by changing the band gap energy required for the system to fluoresce ....

2.14. UV-Vis measurement overlay comparing the CdSe cores (dotted line) against the $\mathrm{CdSe} / \mathrm{ZnS}$ core-shell quantum dots (solid line)

2.15. XRD profile is softened after shelling, suggesting that the $\mathrm{ZnS}$ addition successfully modified the original structure

2.16. FTIR spectrum of the core-shell quantum dots indicates a strong peak at $1710 \mathrm{~cm}^{-1}$, suggesting that the 2-MPA capping agent is carbonyl terminated on the surface

2.17. Fluorescence quenching observed for mercury ions

2.18. Fluorescence quenching observed for cadmium ions

2.19. Fluorescence quenching observed for lead ions

2.20. Intensity behavior as concentration increases differs amongst the three ions, suggesting different mechanisms for interesting with the capping agent and fluorescence quenching

2.21. Fluorescence intensity decreases in both the (left) coating and the (right) quantum dot over time - this brings up the ultimate challenge with quantum dots and that is their limited lifetime, a weakness that must be addressed and optimized for sensing to be effective long-term

3.1. Electrochemical corrosion due to the formation of a cathode at $\mathrm{Fe}_{3} \mathrm{C}$ and an anode at $\mathrm{Fe}$

3.2. Example schematic of corrosion driven by defects caused upon by the presence of an air void from incorrect deposition

3.3. The morphology of the blend is important for describing the impact on barrier properties

3.4. Schematic for incomplete penetration due to excessive surface roughening

3.5. Series of reactions for producing the coprecipitate of zinc and ferric phosphates

3.6. A diverse range of corrosion inhibitors can be used to increase a coating's performance

3.7. Example of a corrosion inhibitor suited for acidic environments

3.8. Example of a corrosion inhibitor suited for alkaline environments 
3.11. Examples of the large number of possibilities of different organic corrosion inhibitors

3.12. Improved quantum yield observed due to surface passivation improving the stability and durability of the quantum dot system

3.13. Addition of TFSI increases quantum yield and optical performance as shown in (a) fluorescence and (b) UV-Vis measurements

3.14. Particle size distribution is more monodisperse after the surface passivation by TFSI

3.15. UV-Vis measurements have greater absorbance due to the increased stability provided by the addition of trioctylphosphine where (a) is without trioctylphosphine and (b) is with the addition of trioctylphosphine

4.1. General summary for the corrosion redox reactions for an iron/water system

6.1. Synthesis roadmap for preparing the cadmium selenide quantum dot cores

6.2. Image of the cadmium source for core synthesis

6.3. Image of the selenium source for core synthesis

6.4. Scale up reaction where the three blue heating mantles are triplicate cadmium sources, and the selenium source is triple the amount in the oil bath

6.5. The reaction flask is quenched in a cold-water bath to stop the cores from growing too large

6.6. A light red color observed after 45 seconds reaction time

6.7. A darker red color observed after 60 seconds reaction time

6.8. A dark burgundy color observed after 75 seconds reaction time

6.9. A side by side comparison of the color differences based on reaction time duration

6.10. The color is far too dark, an almost blood red color, resulting in excessive growth, and cores do not fluoresce

6.11. The raw quantum dot mixture is added into chloroform, but the system is immiscible in the solvent

6.12. Quantum dots fluoresce under a $395 \mathrm{~nm}$ UV source, verifying they were synthesized correctly

6.13. Centrifugation roadmap for purifying the cadmium selenide quantum dot cores

6.14. The raw quantum dot mixture is transferred equally into 6 Falcon centrifuge tubes .... 
6.16. The mixture after addition of $6 \mathrm{~mL}$ acetone

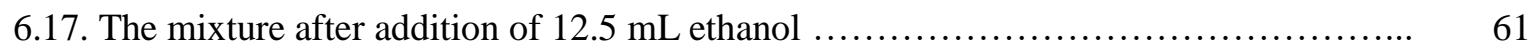

6.18. All 6 Falcon centrifuge tubes loaded into the benchtop centrifuge .................... 62

6.19. The cap was screwed on afterwards tightly using the hand tool before running the centrifuge

6.20. The nanoparticles separated and were stuck to the walls of the centrifuge tube

6.21. The first supernatant was very yellow, indicating collection and purification of raw mixture from impurities

6.22. The second centrifuge had a different, cleaner precipitate fan pattern compared to before

6.23. A comparison of supernatants - the second is considerably clearer and cleaner than the first

6.24. The final precipitate fan pattern had a very clean and consistent gradient throughout all centrifuge tubes

6.25. UV-Vis measurement overlay for the varying reaction times for CdSe cores

6.26. The 45 seconds reaction time mixture is now miscible with chloroform

6.27. The 60 seconds reaction time mixture is now miscible with chloroform

6.28. The 75 seconds reaction time mixture is now miscible with chloroform

6.29. The 45 seconds batch fluoresces a pale-yellow color

6.30. The 60 seconds bath fluoresces a deeper yellow color

6.31. The 75 seconds batch fluoresces a bright orange color

7.1. Synthesis roadmap for shelling the CdSe cores with $\mathrm{ZnS}$ shells

7.2. Benchtop setup with the cores in the middle, leftmost is the $\mathrm{Zn}$ precursor, and rightmost $\mathrm{S}$ precursor

7.3. The CdSe cores flask has an open neck so that the chloroform can escape

7.4. After 1 hour of allowing the chloroform to escape, the volume of the mixture is less than before

7.5. The zinc shelling precursor ready for alternating injection to generate the $\mathrm{ZnS}$ shells

7.6. The sulfur shelling precursor ready for alternating injection to generate the $\mathrm{ZnS}$ shells 
7.7. The finished product with the core-shell $\mathrm{CdSe} / \mathrm{ZnS}$ quantum dots

7.8. The precipitate pattern for the core-shell $\mathrm{CdSe} / \mathrm{ZnS}$ quantum dots after the first centrifuge run is scattered and discontinuous.

7.9. The first supernatant is very yellow compared to washing out the cores alone

7.10. The quantum dots readily separated from the solvents even before centrifuging the sample

7.11. The same phenomena were exhibited with other centrifuge tubes as well

7.12. The $\mathrm{CdSe} / \mathrm{ZnS}$ after purification deposited into chloroform before optical characterization

7.13. A bright orange fluorescence for the CdSe/ZnS under a $395 \mathrm{~nm}$ UV source

7.14. UV-Vis measurements for the CdSe cores before shelling

7.15. UV-Vis measurements for the $\mathrm{CdSe} / \mathrm{ZnS}$ core-shell quantum dots after shelling .......

7.16. UV-Vis measurements comparing before and after shelling - a red shift is expected since shelling increases nanoparticle size

7.17. Fluorescence measurement for the CdSe cores only before shelling

7.18. Fluorescence measurement for the $\mathrm{CdSe} / \mathrm{ZnS}$ core-shell quantum dots after shelling ..

7.19. Fluorescence measurement overlay comparing before and after shelling - a red shift is expected since shelling increases nanoparticle size

8.1. An image of the commercial rust inhibiting topcoat - epoxy enamel

8.2. An image of the commercial Feast \& Watson $2 \mathrm{~K}$ polyurethane

8.3. An image of the industrial use AkzoNobel high solids $2 \mathrm{~K}$ epoxy

8.4. An image of the industrial use PPG Aerospace $2 \mathrm{~K}$ polyurethane 


\section{Quantum Dots as Nanomaterials Used in Coatings}

Core-shell quantum dots have many uses within technology with a diverse portfolio of industries and applications served such as biolabels, sensors, lasers, light emitting diodes, and medicine ${ }^{[1]}$. These materials are fluorescent semiconducting nanocrystals that exhibit zero-dimensional confinement and a characteristic fluorescence when irradiated with a UV source. As nanomaterials, there are several key factors that allow these materials to exhibit such unique and tunable properties.

Nanoscale materials are not subject to the rules and laws that classical physics govern due to their large surface area to volume ratios and changes in intermolecular forces ${ }^{[2]}$. Nanoscale inorganic materials have been integrated into organic coatings to form nanocomposite coatings. The definition of nanomaterials in having large surface area to volume ratios comes into play for these materials due to the interfacial interaction in composite materials. According to ASTM E2456, a nanomaterial is defined as a material that has at least one of its dimensions less than $100 \mathrm{~nm}$ in length ${ }^{[3]}$. The IUPAC does not have a formal definition for the term nanomaterial, but it is generally accepted to follow the definition provided by ASTM E2456. Other governing bodies such as the European Union describe a nanomaterial with an upper limit of $100 \mathrm{~nm}$ and a lower limit of $1 \mathrm{~nm}$. Their definition is more dependent on how the properties change from bulk to nanoscale rather than dimensions alone ${ }^{[4]}$.

Smaller particles will have stronger influences for nanocomposites since their surface bonds are in greater proportion with their small dimensions in participating with these interactions. For example, at a $30 \%$ by volume loading, a $300 \mathrm{~nm}$ nanoparticle with a $10 \mathrm{~nm}$ interfacial layer thickness will have an interfacial material content of $3 \%{ }^{[5]}$. If the particle size decreases to $50 \mathrm{~nm}$, the interfacial material content increases to $22 \%{ }^{[5]}$. For nanocomposites, it is possible that the properties at the interface and that of the nanoparticles become predominant in the composite system. These properties will depend on how the surface bonds interact with the parent material. One example of nanoparticle usage in nanocomposites are nanoparticles integrated into clear coating formulations. Since the particles are so small, they do not readily scatter light 
because they are below the geometric limit of visible light. This results in very little light being scattered, and a reduction in optical performance is therefore not observed.

One example of a property that changes from the bulk to the nanoscale is melting point. Since the bonds are free and cleaved at the nanoscale, less energy is required to melt the system. This is called melting point depression; an example graph is shown in Figure 1.1. Other examples of properties that change include color and appearance, such as how copper transitions from being opaque at the macroscale to transparent at the nanoscale, or phase, such as gold being solid versus liquid at room temperature. Transitioning to smaller and smaller particle sizes - such as nanoparticles like quantum dots - result in unique properties that can be manipulated for use in many diverse applications. A key property that changes at the nanoscale that is of extreme relevance to polymers and coatings is the glass transition temperature. This change occurs due to the surface area to volume ratio in addition to steric and enthalpic effects.

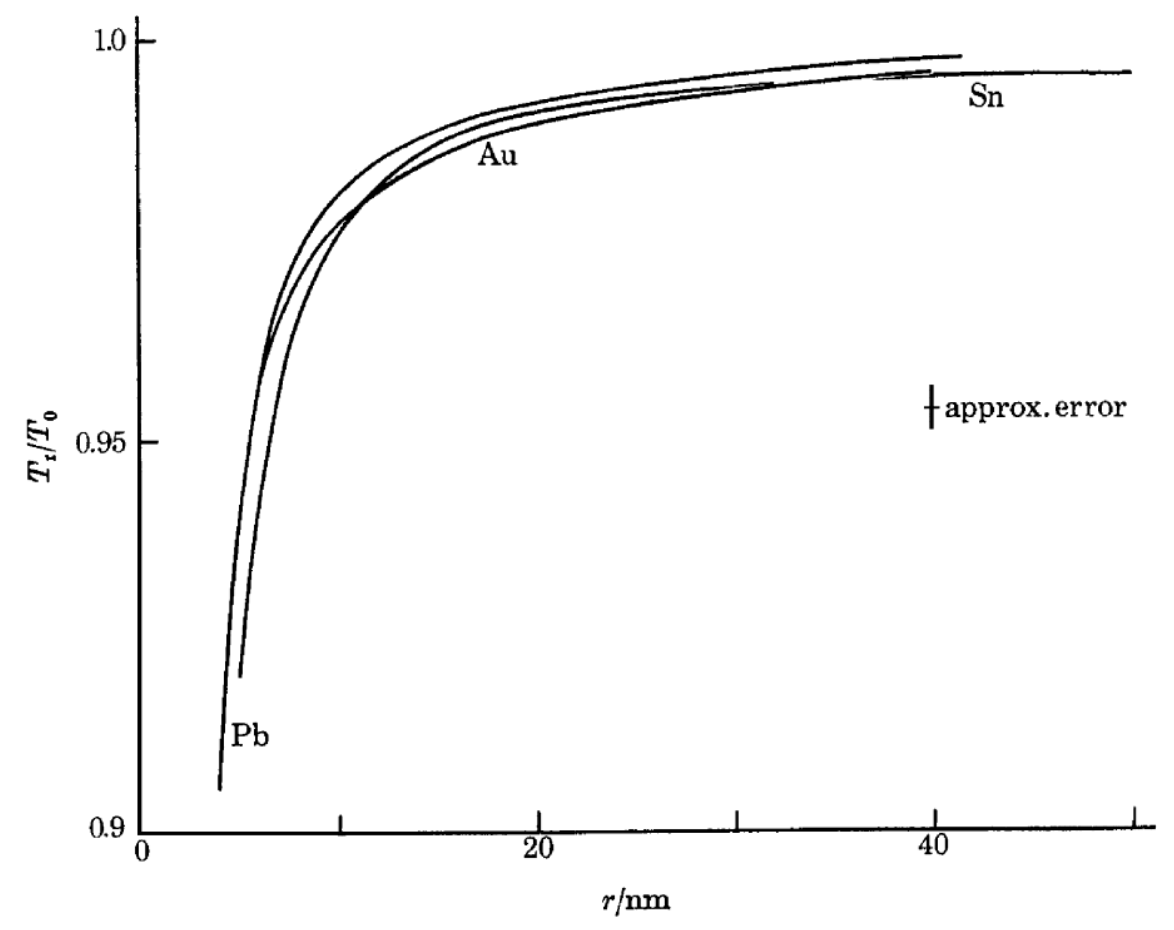

Figure 1.1. Melting point depression occurs approaching the nanoscale due to increased surface energy at smaller dimensions for a variety of metals. ${ }^{[6]}$ 
A major property that must be investigated when considering the integration of nanoparticles into coatings systems is the glass transition temperature $\mathrm{T}_{\mathrm{g}}$. The glass transition is a reversible phase transition from a glassy hard phase to an amorphous soft rubber phase. It can be described as the transition from a disordered solid to that of a disordered liquid. From a kinetics perspective, the glass transition temperature is the temperature at which the local polymer chains begin to move in the polymer. This increased mobility is reflected in the thermal and mechanical properties of the polymer so changes in the heat capacity and stiffness are observed, along with other relevant properties.

Increasing the free volume of a system increases the ease at which these chains can move, thereby decreasing the glass transition. Compositional factors such as having flexible chains or bulky side groups also effects the glass transition, and the addition of plasticizers or copolymers also influences the glass transition. As materials and particles decrease in size and approach the nanoscale, there are expected changes to the glass transition temperature that must be inspected and tailored depending on the target application.

Fryer et al. investigated the effects of how the glass transition temperature changes as the dimensions of a system decrease ${ }^{[7]}$. The purpose of the research was to determine the critical thinness by which the bulk glass transition temperature is no longer expressed in polystyrene and poly(methyl methacrylate) films. The polymers were dissolved in propylene glycol methyl ether acetate and spun cast at $2000 \mathrm{rpm}$ on bare silicon wafers and also on silicon wafers pretreated with hexamethyldisilizane. The films were then annealed for thicknesses in the range of 25 to $650 \mathrm{~nm}$ thick. Glass transition temperature measurements were obtained through differential scanning calorimetry (DSC), and thickness measurements for the thin films obtained through ellipsometry ${ }^{[7]}$. The results of the relationship between the film thickness and glass transition temperature for polystyrene and poly(methyl methacrylate) are shown below in Figure 1.2 and 1.3, respectively. 


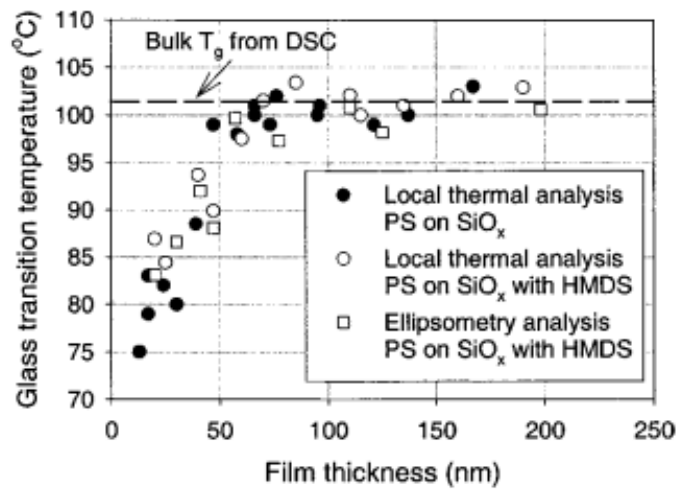

Figure 1.2. The glass transition temperature decreases for polystyrene as it approaches the nanoscale ${ }^{[7]}$.

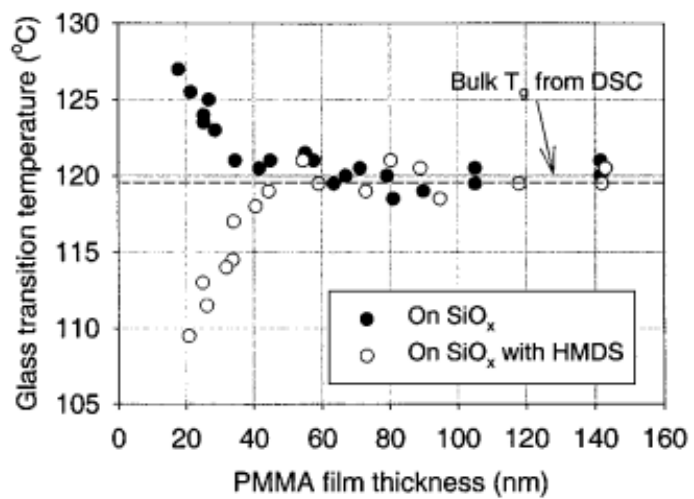

Figure 1.3. The glass transition temperature

decreases with the HMDS treatment due to

intermolecular forces interacting with the

poly(methyl methacrylate) as it approaches the

nanoscale ${ }^{[7]}$.

The results indicate that the glass transition temperature changes as the material decreases in dimensions, verifying that the material exhibits new nanoscale properties after a critical length. However, it is interesting to note that the glass transition temperature decreased in the polystyrene system, whereas in the poly(methyl methacrylate) system, it increased and decreased depending on the substrate. This was explained in that the polarity and that the glass transition temperature is not supported by a nonpolar substrate. The intermolecular forces and increased surface energy for a nanoscale material interact readily with the substrate, thus defining the polarity of the substrate is of vital importance. As a result, the hexamethyldisilizane treated wafer exhibited a decrease in glass transition temperature.

Jiang et al. also contributed to the changes in glass transition temperature observed as the length scales decrease ${ }^{[8]}$. Looking at the thickness of the thin films, Jiang reaffirms the importance of investigating the interface between the polymer composing the thin film and the substrate material. The bonding at this interface is crucial in determining whether the materials properties - in this case, the glass transition temperature - is reinforced or hindered ${ }^{[8]}$. 
The importance of the substrate is shown in comparing Figure 1.4 and 1.5 below, where the glass transition temperature $\mathrm{T}_{\mathrm{g}}$ is plotted against the diameter $\mathrm{D}$ of the thin film. The former is that of free-standing polystyrene films while the latter represents polystyrene films deposited onto a silicon substrate pretreated to have hydrogen bonding ${ }^{[8]}$. Like the results reported by the research conducted by Fryer et al., the substrate and its interactions with the polymer are important for determining how the glass transition temperature changes at the nanoscale.

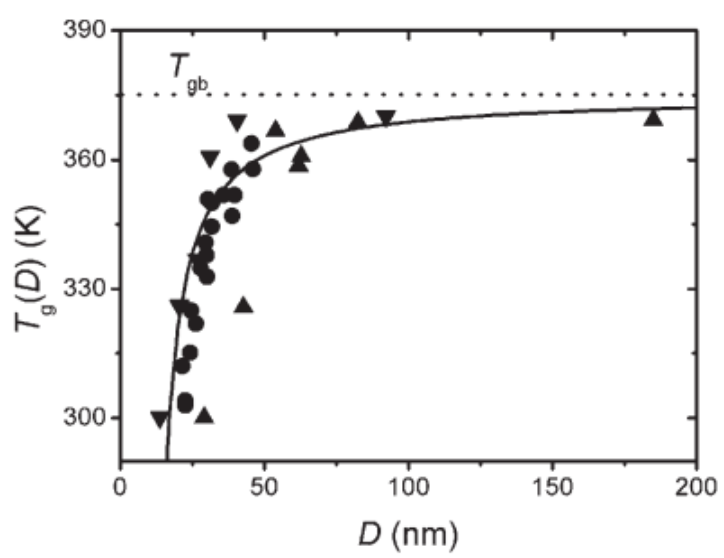

Figure 1.4. The glass transition temperature decreases for free-standing polystyrene thin films at the nanoscale ${ }^{[8]}$.

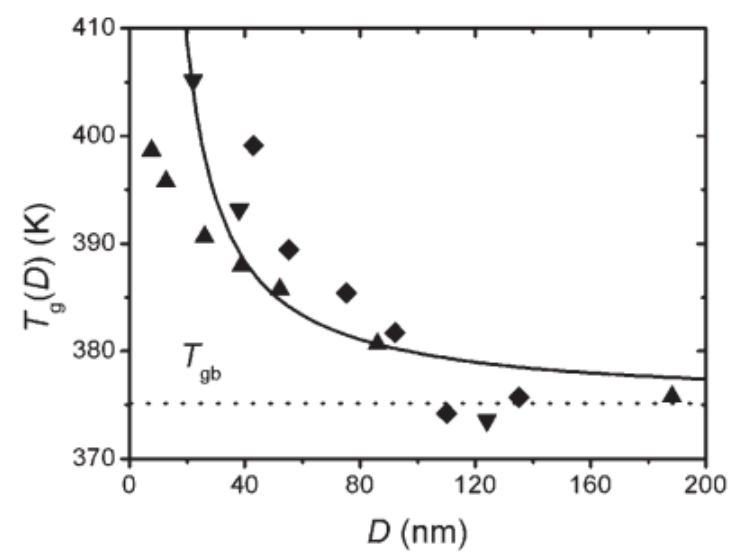

Figure 1.5. The glass transition temperature increases for polystyrene thin films supported by hydrogen bonding on a silicon wafer at the nanoscale ${ }^{[8]}$.

These results support the theorem that many properties of a material will change upon becoming smaller and smaller and moving into the nanoscale. For nanoparticles embedded into coatings, both systems must be examined thoroughly to investigate how these properties change. In addition to increasing or decreasing the glass transition temperature, it is also possible for a system to have no effect on it as well, as reported by Clarke et al. with alumina and silica nanoparticles having no effect for a polyurethane film ${ }^{[9]}$. Other combinations of polymers and substrates have also been reported in literature such as alumina/PMMA, polyurethane/silica, polyurethane/organoclay, and polyurethane/montmorillonite. 
One of the earliest contributions for nanomaterials embedded into coatings systems was brought forth by Toyota Motor Company’s work on nylon/nanoclay composites ${ }^{[10]}$. This system had montmorillonite nylon composites that provided flame resistance along with improving the mechanical properties of nylon-6 including stiffness, yield strength, and impact resistance ${ }^{[10]}$. The issue here with nanoclays is that they are difficult to integrate into coatings systems due to their structure and shape. Often irregularly shaped, nanoclays require complex processing conditions that are friendly to typical coating mixing operations. Nanoclays often must be chemically and physically treated to transition from their aggregated intercalated state to an exfoliated state before being ready to mix with coatings.

The intercalated state can be described as when a single polymer chain of the nanoclay is embedded between layers of the composite material. The intercalated structure is not ideal because the nanoclays are not dispersed evenly throughout the composite, resulting in reduced performance. Instead, these materials are surface treated with quaternary ammonium compounds and other coupling agents to exfoliate and separate them into individual layers ${ }^{[10]}$. This also improves processability and maximizes the benefits of integrating nanoclays into their parent nanocomposite. An example of these two configurations is shown in Figure 1.6.

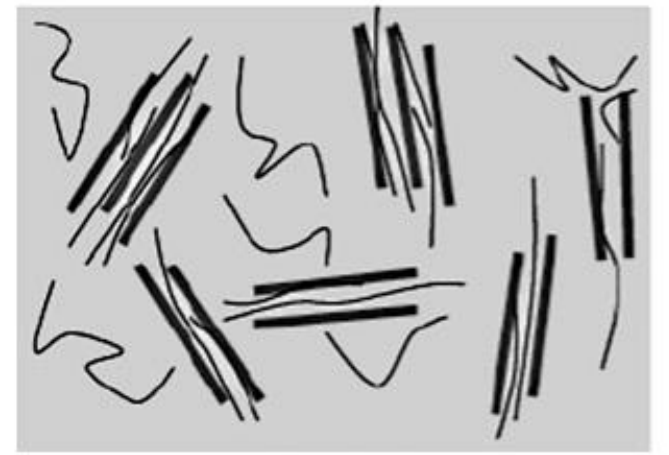

Intercalated

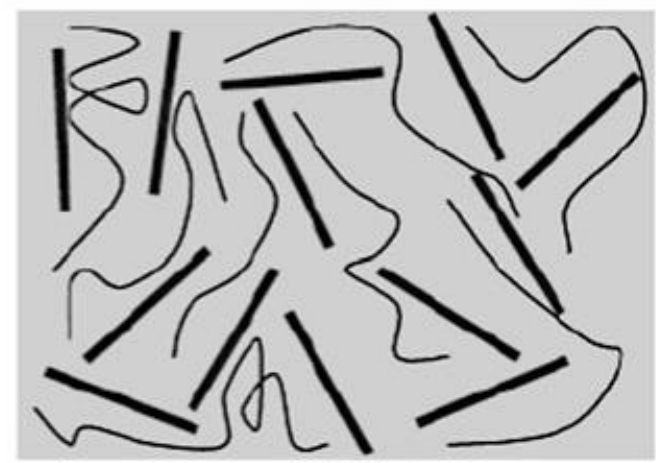

Exfoliated

Figure 1.6. Nanoclays must be surface treated to transition from the intercalated to exfoliated state to maximize dispersion and performance increase in the nanocomposite ${ }^{[10]}$. 
Quantum dots, on the other hand, are assumed to be spherical in size and thus do not have the same concerns in packing inefficiency. However, the same issue arises in that there must be considerable focus on the dispersion of nanomaterials within a coating. Outside of quantum dots, some examples of nanoparticles that have been historically used in coatings are shown in Figure 1.7.

\begin{tabular}{|l|l|}
\hline \multicolumn{1}{|c|}{ Coating Property } & \multicolumn{1}{c|}{ Nanomaterial } \\
\hline Anti-microbial & $\mathrm{CuO} ; \mathrm{TiO}_{2} ; \mathrm{ZnO}$ \\
\hline Gas Barrier & Nanoclays \\
\hline Corrosion & Nanoclays, boehmite \\
\hline $\begin{array}{l}\text { Electrical Conductivity, } \\
\text { Static Charge Dissipation }\end{array}$ & $\mathrm{ITO}, \mathrm{ATO}, \mathrm{SnO}_{2}$ \\
\hline Fire Retardant & Nanoclays \\
\hline IR-Absorption/Reflection & $\mathrm{ITO}_{2} \mathrm{ATO}, \mathrm{TiO}_{2}, \mathrm{In}_{2} \mathrm{O}_{3}$ \\
\hline Magnetic & $\mathrm{Fe}_{2} \mathrm{O}_{3}$ \\
\hline $\begin{array}{l}\text { Mechanical, Scratch } \\
\text { Resistance }\end{array}$ & $\mathrm{Al}_{2} \mathrm{O}_{3} ; \mathrm{SiO}_{2} ; \mathrm{ZrO}_{2}$ \\
\hline Photocatalysis, self-cleaning & $\mathrm{TiO}_{2} ; \mathrm{ZnO}$ \\
\hline UV stability & $\mathrm{TiO}_{2} ; \mathrm{ZnO} ; \mathrm{BaSO}_{4} ; \mathrm{CeO}_{2}$ \\
\hline
\end{tabular}

Figure 1.7. Examples of nanoparticles and their properties that can be embedded into coatings systems

Silica colloids have long been implemented into clear coatings to improve abrasion and scratch resistance. This can be observed in many industrial and commercial applications such as use in automotive top coats and acrylic lenses used for eyeglasses. High silica loading levels can be difficult to achieve with silica colloids because they will lead to haziness and a lack of optical clarity.

Applications like these require a large difference between the refractive indices of the nanomaterials and the substrate. The small size of nanoparticles also promotes less scattering of light. Some nanoparticles of interest include $\mathrm{SiO}_{2}, \mathrm{ZrO}_{2}$, and $\mathrm{Al}_{2} \mathrm{O}_{3}$. In addition to reinforcing scratch and abrasion resistance, these materials can also increase the mechanical and thermal properties of the system.

Nanomaterials have had a long history of integration into coatings systems. Nanomaterials have special and unique properties due to their large surface area to volume ratios, increased surface energies, and do not obey classical physics. Some properties that exhibit radical changes include melting point temperature, 
glass transition temperature, and mechanical properties like hardness and stiffness. Nanomaterials can also act as performance additives in providing new properties such as flame retardance, improved color and gloss, and UV stability.

A special type of nanomaterial that has not yet been discussed are a class of nanomaterials called quantum dots. This type of material is a semiconductor nanocrystal material that fluoresces a characteristic wavelength dependent upon the size of the particle. The following section will briefly introduce quantum dot systems before discussing the physics and working mechanism for how they operate. 


\section{$\underline{2 \text { Quantum Dot Basics }}$}

Quantum dot materials have usage in a wide variety of applications serving industries related to sensor technology such as biomarkers and labeling in biological organisms to usage in optical engineering with applications in laser technology or in supplementing light emitting diodes ${ }^{[1]}$. All quantum dot materials can be classified as semiconducting nanoparticles that exhibit a characteristic fluorescence based on the unique properties that arise from their large surface area to volume ratios which result in a large proportion of surface bonds and accompanying large surface energies dominating over bulk properties.

Nanoscale materials are not subject to the rules and laws that classical physics govern due to their large surface area to volume ratios and changes in intermolecular forces at that scale ${ }^{[1]}$. The surface area to volume is inversely proportional to the particle size so these considerably smaller particles have new properties different than the bulk properties and instead adhere to quantum physics rather than classical physics.

Consider Figure 2.1 below which shows arrangements of cubes at different dimensions. Assuming each side of the smaller cubes is $1 \mathrm{~cm}$, the smallest cube in (a) has a surface area of $6 \mathrm{~cm}^{2}$ and a volume of $1 \mathrm{~cm}^{3}$ compared to $24 \mathrm{~cm}^{2}$ and $8 \mathrm{~cm}^{3}$ for (b). For (c), the surface area would be $54 \mathrm{~cm}^{2}$, and the volume would be $27 \mathrm{~cm}^{3}$. In calculating the surface area to volume ratio, (a) is $6: 1$, (b) is $3: 1$, and (c) is $2: 1$. As the material becomes smaller and smaller, total surface area increases greatly while the volume decreases, resulting in large surface area to volume ratios. For example, a $1 \mu \mathrm{m}$ sphere will have a surface area to volume ratio of 6:1 whereas a $3 \mu \mathrm{m}$ sphere will have a 2:1 ratio. These ratios will increase to even larger numbers as the diameters approach the nanoscale. This leads to unique nanoscale properties such as changes in mechanical properties, thermal properties, and even chemical reactivity. 


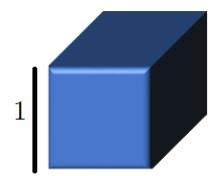

(a)

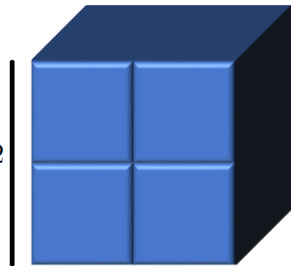

(b)

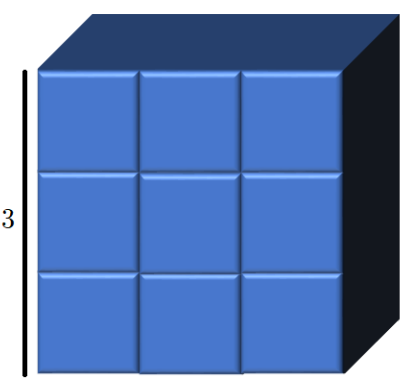

(c)

Figure 2.1 Surface area to volume ratios increase as materials become smaller; here (a) has a surface area to volume ratio of $6: 1$ while (b) is $3: 1$ and (c) is 2:1.

\section{$\underline{2.1 \text { Band Gap Energy and Density of States }}$}

Traditional semiconducting materials have a characteristic bandgap that quantifies the excited energy that must be provided into the system to excite an electron from the valence band to the conduction band. Represented as the band gap energy $\mathrm{E}_{\mathrm{g}}$, exciting an electron from one band to the other forms an electronhole pair ${ }^{[1]}$. In Figure 2.2 below, the valence band is depicted by the green box and above it is the conduction band in red. The band gap is shown in purple, and its value changes when comparing the different semiconductor materials. 


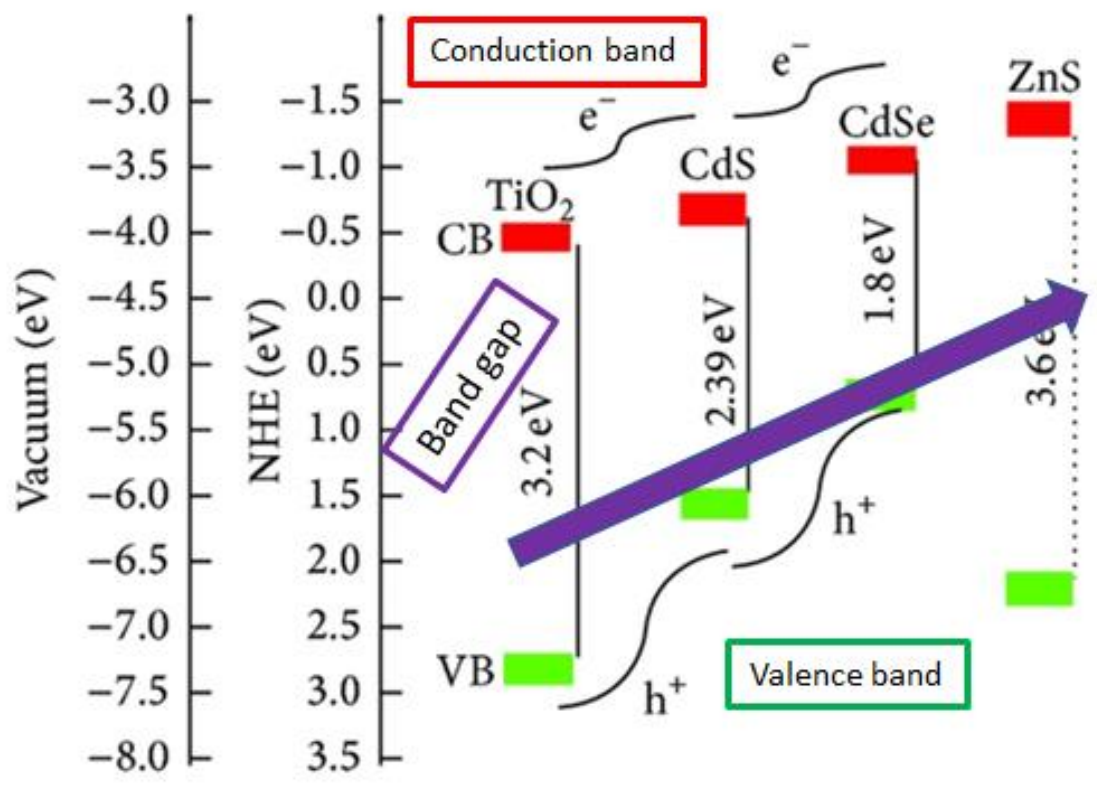

Figure 2.2. An electron-hole pair is generated when enough energy is provided into the system to excite an electron from the valence band to the conduction band-different materials require different energy as the band gap.

For a bulk material, as more and more energy is input into the system, the density of states for those electrons is continuous ${ }^{[1]}$. This means that the number of excited electrons from valence to conduction and therefore electron-hole pairs - increases and is variably proportional to the input energy, suggesting that there is no confinement for these electron pairs due to their relatively large bulk dimensions. In Figure 2.3, $\mathrm{D}(\mathrm{E})$ represents the density of states as a function of input energy into the system, which allows researchers to predict the level of confinement for the electron-hole pairs. For quantum dots, there is zerodimensional confinement, resulting in the electron-hole pairs becoming quantized and only appearing at specific energy inputs.

An analogy to describe quantum confinement is a spherical particle placed inside a cube. At large dimensions, this particle can bounce off all interior faces of the cube and is free to rebound in any combination of three dimensions. As the cube shrinks, the particle loses this directional flexibility and may 
have its movement constrained to one dimension. At the extreme nanoscale such as the case with quantum dot materials, it cannot move at all and is thus zero-dimension confined.

Confinement can further be observed in Figure 1.4, where going from (a) to (d), it is observed that dimensionality increases. In (d), the location and confinement of an electron-hole pair is continuous in all three directions, meaning that it can exist anywhere within the bulk material using a description of those three planes - for example, an electron-hole pair can be located at $(\mathrm{x}, \mathrm{y}, \mathrm{z})$ in Cartesian coordinates. Compare this to (c), where due to the size of the structure, it can only exist in two dimensions - (x,y) if using Cartesian coordinates. Similarly, in (b), the location can only be described in one axis. For quantum dots, the behavior in (a) is observed, where it will exist at one specific energy and location. The confinement of these density of states allows for a more discrete and quantized description of the position of the electron-hole pair in the system.

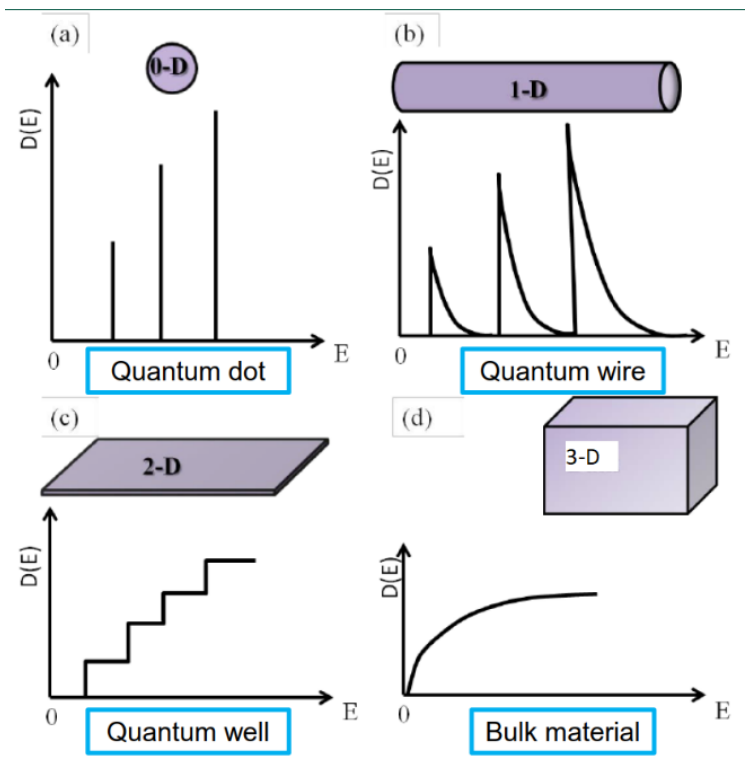

Figure 2.3. Confinement refers to restricting the possible degrees of freedom in which the electron-hole pair can be located - as particle size decreases, dimensionality decreases and exists as a discrete quantized energy level; dimensionality is observed as (a) 0-D, (b) 1-D, (c) 2-D, and (d) 3-D. ${ }^{[1]}$ 
As nanoparticles, quantum dots are just like other nanomaterials in that the size and scaling of their particles provides unique and interesting properties that can be manipulated and adapted for use in several technologies ${ }^{[1]}$. These unique properties arise due to the large surface area-to-volume ratios of these nanoparticles. The excess of surface bonds gives results in radical changes in materials properties such as mechanical and thermal properties or chemical reactivity. For the case of quantum dot materials and the scope of this research, the overarching importance lies in the color that is fluoresced when the quantum dot is irradiated by a $395 \mathrm{~nm}$ UV source.

As shown in Figure 2.4, particle size is everything, especially at the nanoscale and for quantum dot materials. As the size of the nanoparticle increases, the fluoresced color increases in wavelength as the band gap decreases, resulting in the emission of a lower energy and longer wavelength. Past a certain critical maximum, the nanoparticles will no longer exist at the nanoscale, and therefore, they will no longer fluoresce under irradiation. This is a key concept for the reaction portion of this thesis, where the reaction time is vital for determining the nucleation and growth of the quantum dot particles. There is considerable interest in tailoring the size of the quantum dots for applications where color reproduction is important, such as usage in optical applications like lasers, display technology, and light emitting diodes. For this research, the size is important; however, more significant is that the reaction is successful in reproducibly synthesizing working quantum dots. Additionally, Figures 2.5-2.8 below also demonstrate the differences in the color fluoresced, with the latter images representative of larger and larger nanoparticle sizes. 


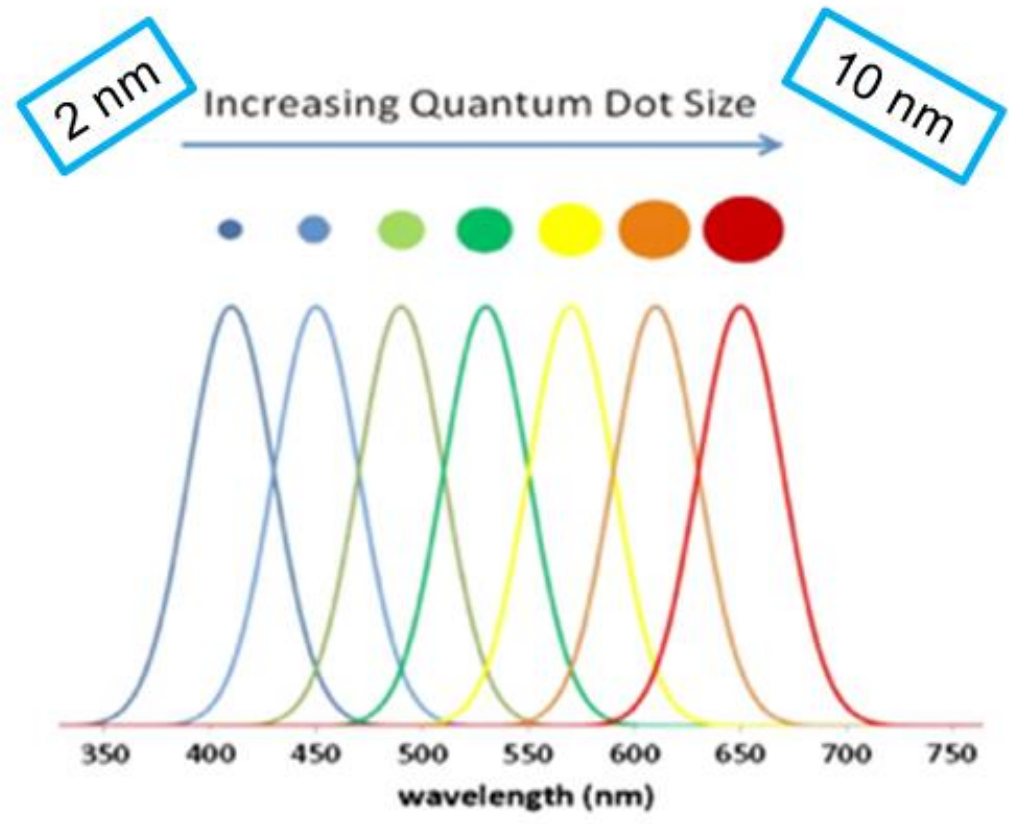

Figure 2.4. As quantum dot nanoparticles become larger, the color fluoresced changes, represented as larger and larger wavelengths in the visible light spectrum. ${ }^{[10]}$ 


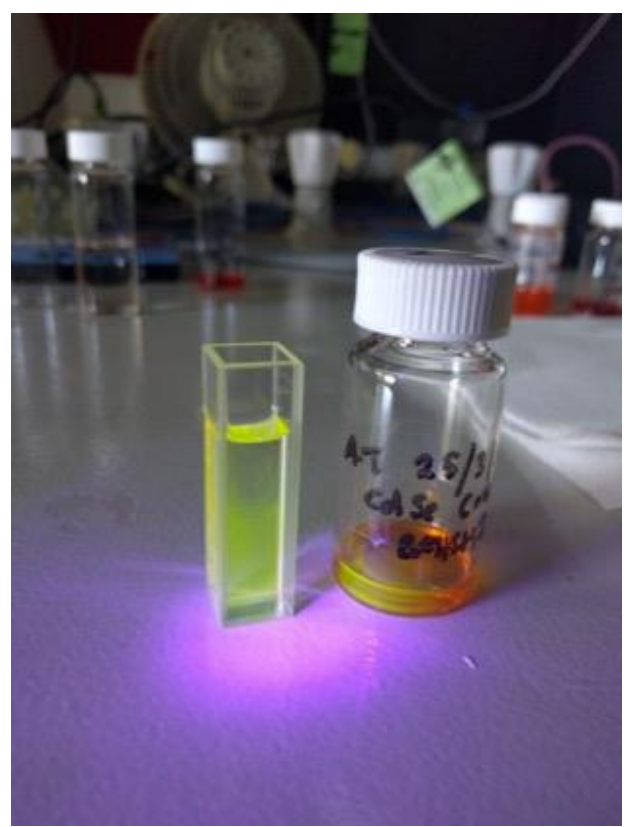

Figure 2.5. Green color $-525 \mathrm{~nm}$.

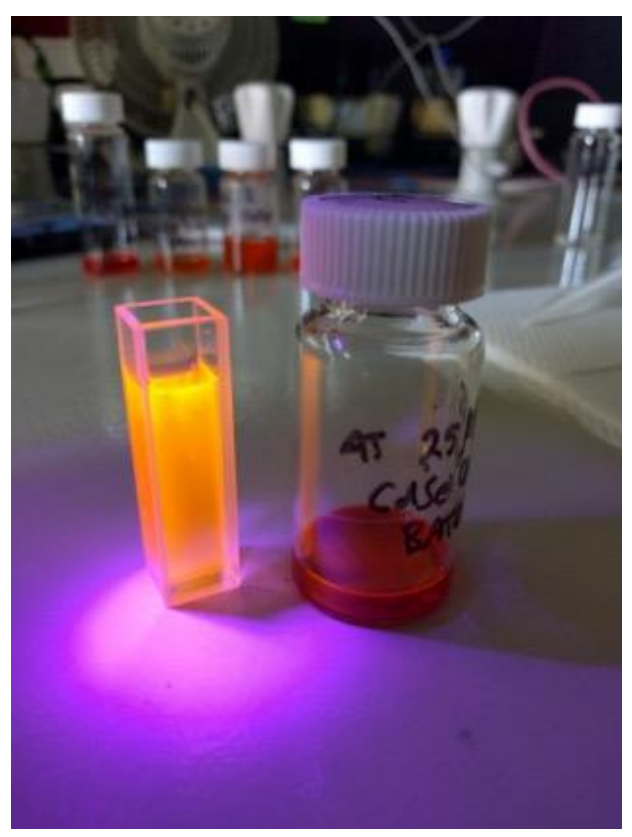

Figure 2.7. Orange color $-610 \mathrm{~nm}$.

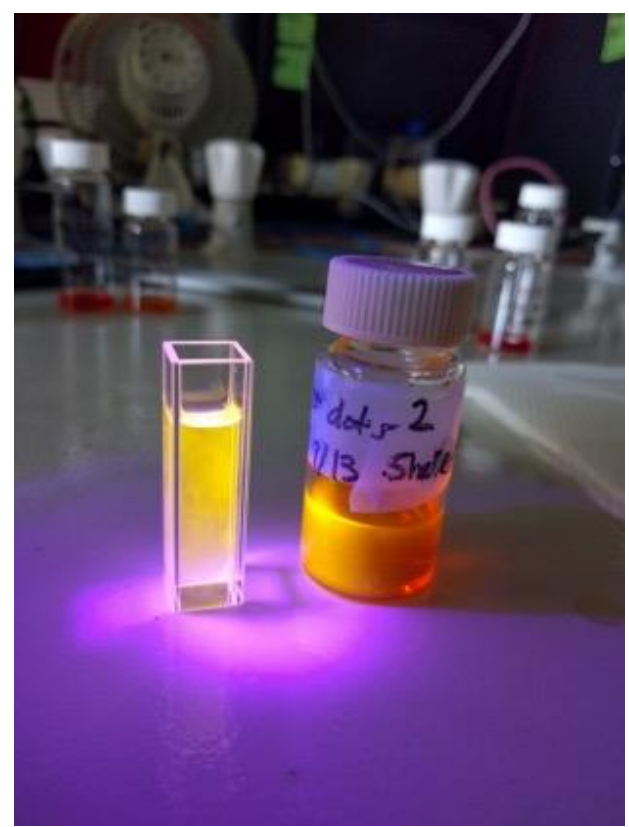

Figure 2.6. Light yellow color $-575 \mathrm{~nm}$.

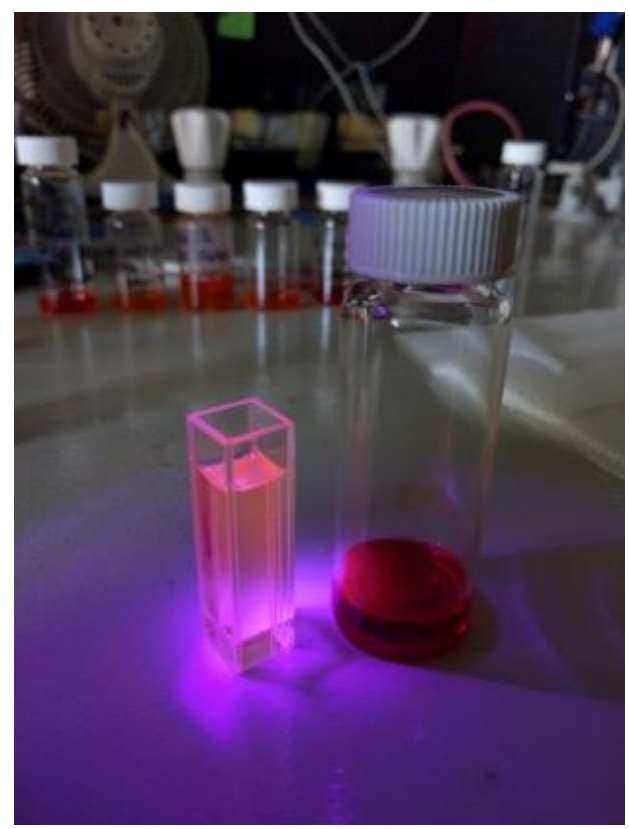

Figure 2.8. Light red color $-650 \mathrm{~nm}$. 
Quantum dots have a large range of flexibility when tailoring the wavelength of light that is to be fluoresced. For the purpose of this research, the fluoresced color is not as important as the fluorescence quenching effect, however this does not discredit the use of quantum dots for alternative applications where manipulating and controlling the fluoresced color is important.

\subsection{Physics Behind Core-Shell Quantum Dots}

Quantum dots by themselves are made of individual elements that contribute to traditional semiconducting materials. These materials are often Group II-VI, IV-VI, and III-V elements in the periodic table. Examples of common quantum dot configurations include $\mathrm{CdS}, \mathrm{ZnS}, \mathrm{CdSe}$, and InAs ${ }^{[1]}$. These quantum dots by themselves are referred to as cores. Shelling is a chemical procedure by which the core is covered entirely on its surface by another compound, typically for enhanced stability and durability. Regarding nomenclature, core-shell quantum dots are described with a forward slash separating the core from the shell respectively; CdSe/ZnTe therefore describes a cadmium selenide core with a zinc telluride shell. This nomenclature can also be expanded for the presence of multiple shells. For example, CdSe/ZnS/ZnS/ZnS would be a cadmium selenide core with three zinc sulfide shells protecting it. In this configuration, each $\mathrm{ZnS}$ shell is synthesized and constructed over the previous. A schematic of the core-shell configuration is shown in Figure 2.9. 


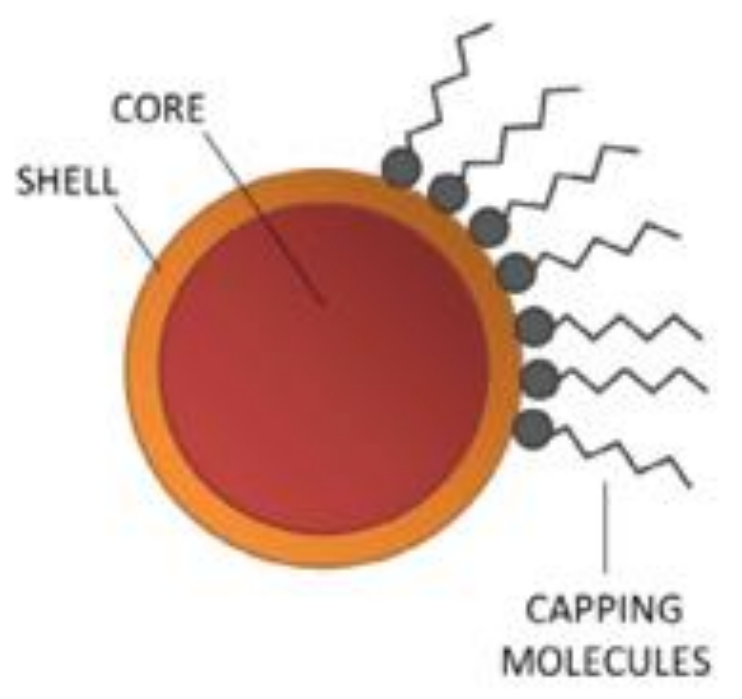

Figure 2.9. Schematic of a core-shell quantum dot with attached capping molecules. ${ }^{[1]}$

In addition to providing improved stability and optical performance via shelling, quantum dot cores can also be modified with the addition of capping agents or capping molecules. This type of agent chemically adheres or reacts onto the surface of the quantum dot - shelled or not shelled - resulting in the reactivity at the surface being altered. One example of a capping molecule is traditional surfactants used to stabilized latex particles. In this example, the surfactant will act to mediate and control the particle size of the quantum dot nanoparticle during particle growth. This improves the processability of the material by preventing the material from growing too large and transitioning from the nanoscale to the macroscale and therefore exhibit bulk material properties.

Surfactant molecules and capping agents that perform a similar role in protecting the quantum dot cores are often organic compounds ${ }^{[1]}$. To supplement the inorganic shells surrounding the quantum dot cores, inorganic capping agents can also be used to perform a similar role. In this case, there is no reaction with the shells, and instead, the inorganic capping agent can act as both an extra additional shell layer but also as a pseudo nanoparticle - therefore, it can be observed that there is a core-shell quantum dot surrounded by another quantum dot. This presents an interesting configuration where the external capping agent also has a band gap energy, therefore creating a band gap "nanoparticle sandwich" between the core and the 
protective surrounding agent ${ }^{[1]}$. The nanoparticle sandwich is present because of differing band gap energies between the layers. This sort of structure can be useful when the core is surrounded by a shell that has a greater band gap energy, resulting in higher quantum yield since the core will be fluoresced while the shell will not. Figure 2.10 below suggests different configurations of core-shell quantum dots - the addition of an inorganic capping agent outside the shell provides an additional layer to the nanoparticle sandwich.

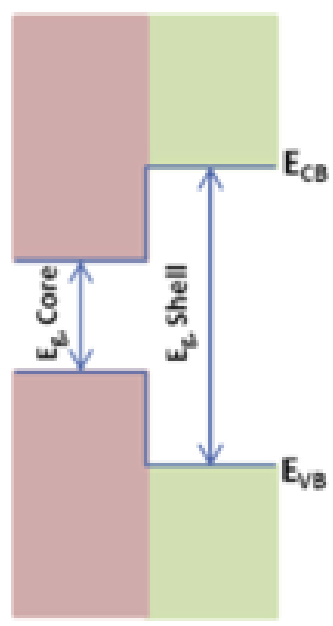

TYPE I

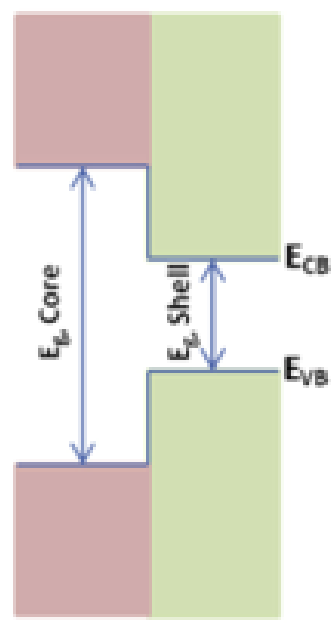

INVERSE TYPE I

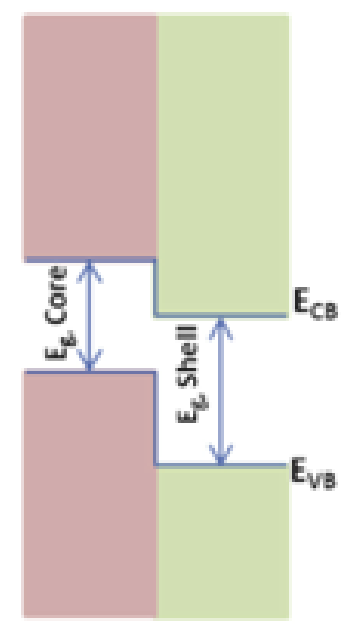

TYPE ॥

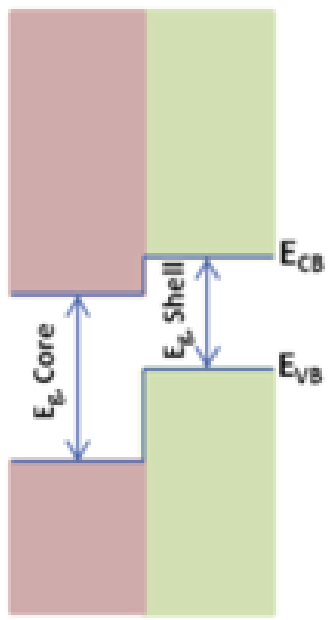

INVERSE TYPE II

Figure 2.10. Various types of configurations for systems where there are multiple band gaps - core-shell quantum dots generally focus on Type I systems to optimize quantum yield and performance. [10]

The importance of a nanoparticle sandwich for improving quantum yield and optical performance is dependent upon the structure and configuration of the band gaps of the layers. Having a system where the inorganic capping agent has a larger band gap than the core-shell system will provide increased photostability and higher quantum yield since the energy required to excite the core-shell will be only sufficient to excite the interior core, minimizing any losses in the system due to recombination events by the electron-hole pairs.

Since the exterior shell or capping agent is not excited since there is insufficient energy capable of exciting it, all energy and excitation only occurs at the interior core. The sandwich effectively locks and constrains 
the interior core-shell structure and its optical characterization response with a final exterior coating that traps the signal inside. The most common example of a core-shell quantum dot flanked with an inorganic capping agent that exhibits this trapping and containment of quantum yield is a $\mathrm{CdS} / \mathrm{HgS} / \mathrm{CdS}$ system. Often, metals and metal oxides such as $\mathrm{Ni}, \mathrm{Co}$, and Fe can be utilized as additional shell layers over the cores as inorganic capping agents ${ }^{[1]}$.

Ultimately, the presence of shells and capping agents are crucial for improving the durability and overall photostability for optical performance for quantum dot core materials. The brief discussion on the usage of organic capping agents such as surfactants or those from the inorganic family such as metals and metal oxides suggest that there is plenty of room for research to be conducted for these agents to operate and improve the performance of quantum dot materials.

Improving the performance of the quantum dot is enhanced along with integrating the properties of the capping agents, resulting in increased strength, colloidal stability, and resistance to environmentally driven reactions such as oxidation and reduction reactions. Specific capping agents are also integral for having these quantum dot systems soluble or dispersible in media of different polarity ${ }^{[11]}$. Considering all of this, the usage of capping agents can be integrated into this research to further improve the quantum yield and optical performance of these quantum dot systems.

\section{$\underline{2.3 \text { Quantum Dots as Sensing Materials }}$}

Quantum dot materials have risen in popularity and are prominently utilized for sensing applications due to how their characteristic fluorescence can be quenched to detect the presence of specific analytes ${ }^{[1]}$. By manipulating the capping agents or the shells in a core-shell quantum dot material to react with a specific analyte, the normalized fluorescence will decrease as this reaction proceeds. One example is a CdSe/ZnS system conjugated with glucose oxidase and horseradish peroxidase to sense the presence of glucose. The organic capping agents in this scenario are the enzymes that will react with glucose, and the successful breakdown of glucose by these agents removes them from the surface of the quantum dot, therefore 
collapsing the structure and decreasing the stability of the nanoparticle. As more and more glucose are broken down, an increasing amount of the capping agent is removed from the quantum dot, resulting in its stability decreasing. This is shown in Figure 2.11 below.
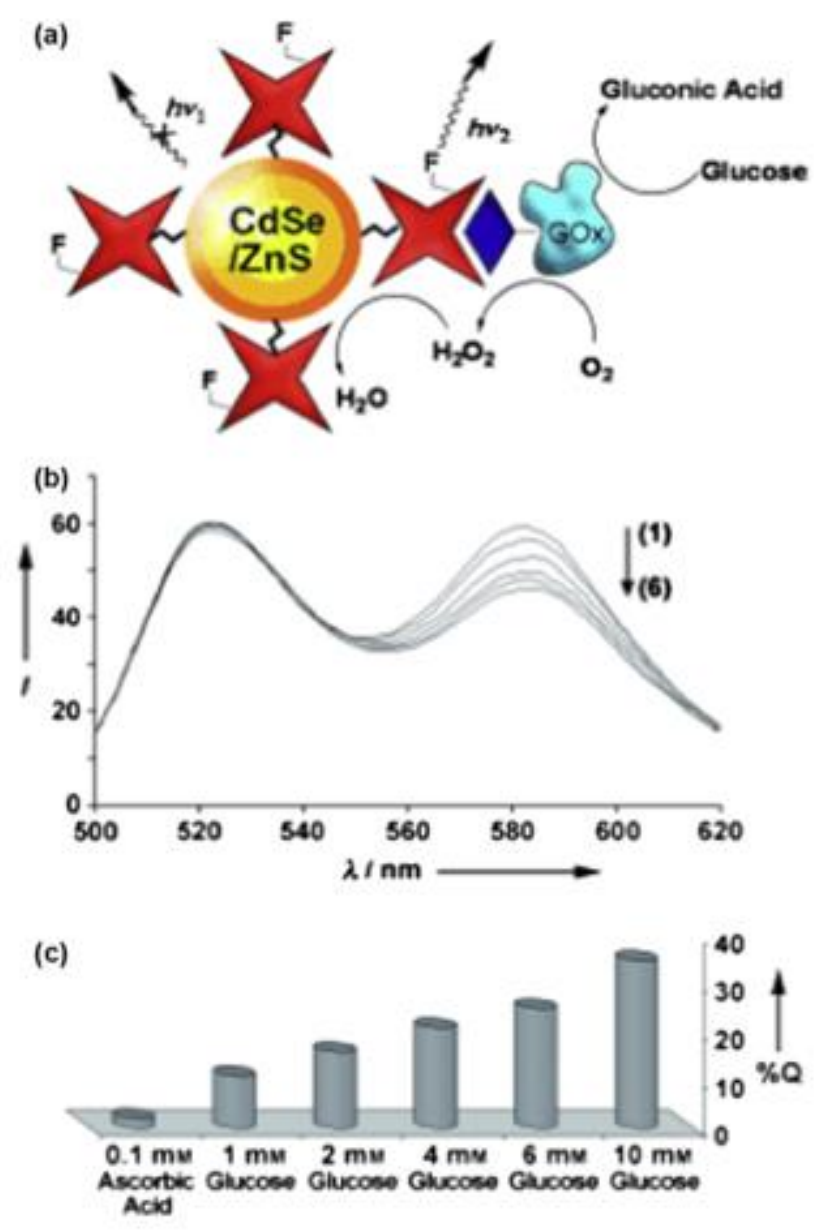

Figure 2.11. Example of quantum dot for sensing application: (a) schematic of glucose oxidase reacting with glucose, (b) fluorescence quenching as concentration of glucose increases, (c) percent quenched for standard glucose solution added. ${ }^{[1]}$

Fluorescence quenching can also be utilized for other responses, such as $\mathrm{pH}$ stability ${ }^{[1]}$. In this case, the attachment of $\mathrm{pH}$ sensitive ring compounds to a parent structure responds to external media by opening at different $\mathrm{pH}$ environments. In this case, the attached capping agent is a ring structure in the form of 1,3oxazine that is acid or base stimulated. As the ring opens, it creates a new structure that acts as a 
chromophore called 4-nitrophenylazophenol ${ }^{[1]}$. This particle does not absorb in the visible region so therefore the fluorescence is quenched. Other fluorescence quenching applications that operate for sensing include detecting urea, tetra-alkylammonium halides, hydrogen sulfate, and gases such as argon, air, and oxygen. A summary of fluorescence quenching and its linearity with concentration is shown in Figure 2.12 below.
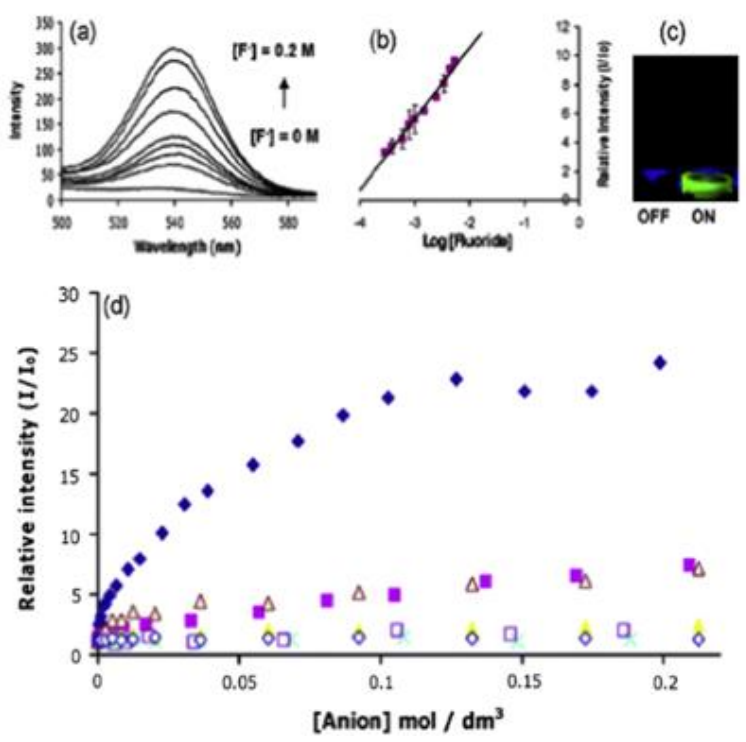

Figure 2.12. Quantum dot sensing application where the attached ring structure is $p H$ sensitive - (a) fluorescence quenching as fluoride concentration is increased, (b) plot of relative intensity of fluorescence against log [fluoride], (c) image of "on" and "off" states, and (d) plot of relative intensity against concentration for 5 titrated against fluoride stock solution. ${ }^{[1]}$

Ultimately, the presence of any analyte that can react with the capping agents or the shell itself of a coreshell quantum dot will collapse the structure and decrease the stability of the quantum dot nanoparticle. The resulting fluorescence will then decrease and quench, supporting the assignment that these analytes reactive or unreactive - are present. Fluorescence quenching operates based on stability. Disruption of the 
stability of the quantum dot material will dampen and decrease the intensity of the characteristic fluorescence, as shown below in Figure 2.13.

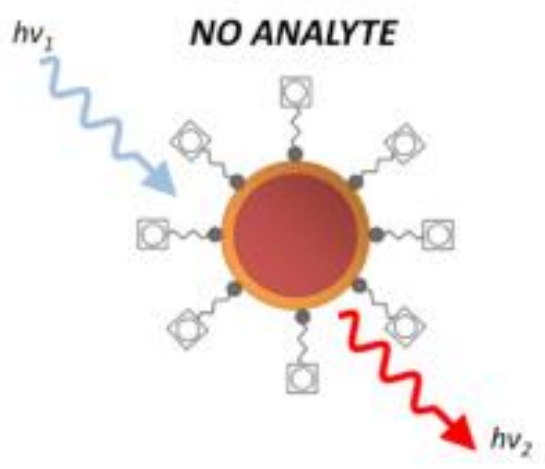

Fluorescence

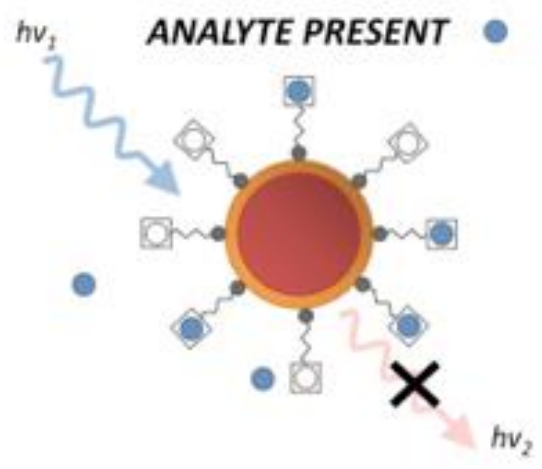

Quenching

Figure 2.13. The presence of an appropriate analyte interacts with the capping agent on the surface of the quantum dot system, thereby disrupting the characteristic fluorescence and decreasing its intensity by changing the band gap energy required for the system to fluoresce. ${ }^{[1]}$

\subsection{Fluorescence Quenching to Detect Heavy Metal Ions}

Vasudevan et al. have reported the detection of heavy metal cations using core-shell CdSe/ZnS quantum dots ${ }^{[12]}$. The focus of this study is the detection of heavy metal cations present in water systems for environmental monitoring and toxicology purposes. Water quality and purity are of utmost importance especially for the detection of heavy metals such as lead, mercury, and cadmium - all of which are toxicological hazards to the human body and many animals and other living organisms. In this research, the capping agent that was utilized was 2-mercaptopropionic acid, and the quantum dot system in question was modified by a phase-transfer agent to be dispersible in water through a phase transfer reaction ${ }^{[12]}$.

Figure 2.14 is a UV-Vis measurement of the synthesized cores and the shelled cores. The red shift in the wavelengths between the two measurements verifies that confinement occurred, and the longer wavelength also suggests there is a larger particle size which is confirmed with the increased radius provided from the shelling over the core itself. 


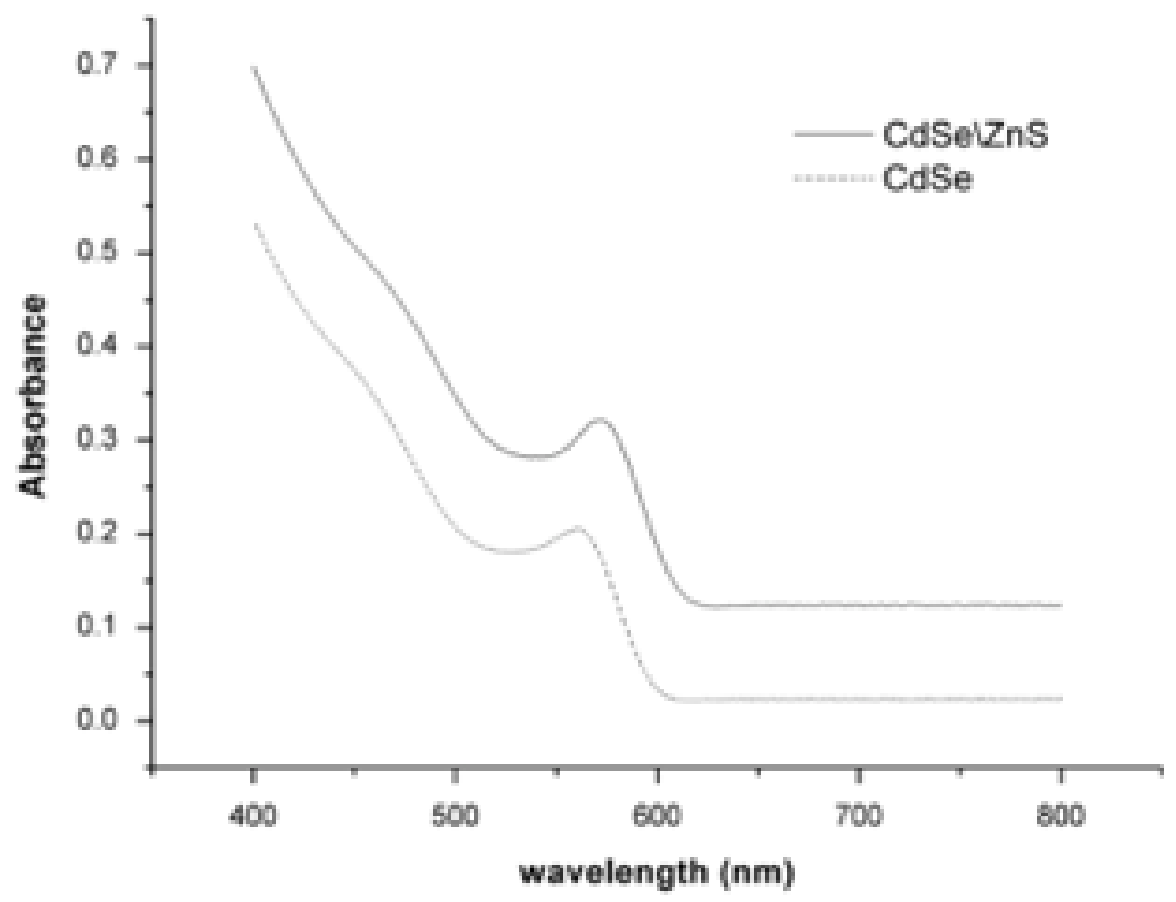

Figure 2.14. UV-Vis measurement overlay comparing the CdSe cores (dotted line) against the CdSe/ZnS core-shell quantum dots (solid line). ${ }^{[12]}$

The XRD profile of the shelled and 2-MPA capped CdSe quantum dot structure is also provided in Figure

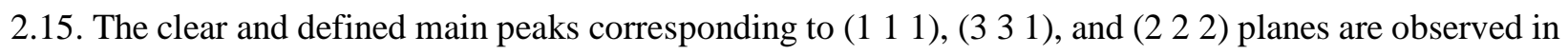
the pure parent $\mathrm{CdSe}$ structure, with the addition of the $\mathrm{ZnS}$ shells disrupting the sharp peaks. Additionally, the shelling by $\mathrm{ZnS}$ and additional surface modification by attaching 2-MPA does not create any new peaks, suggesting that the process was successful. This also suggests that there is no free $\mathrm{ZnS}$ or 2-MPA that is unreacted in the system, also supporting that the reaction was conducted in an ideal manner ${ }^{[12]}$. 


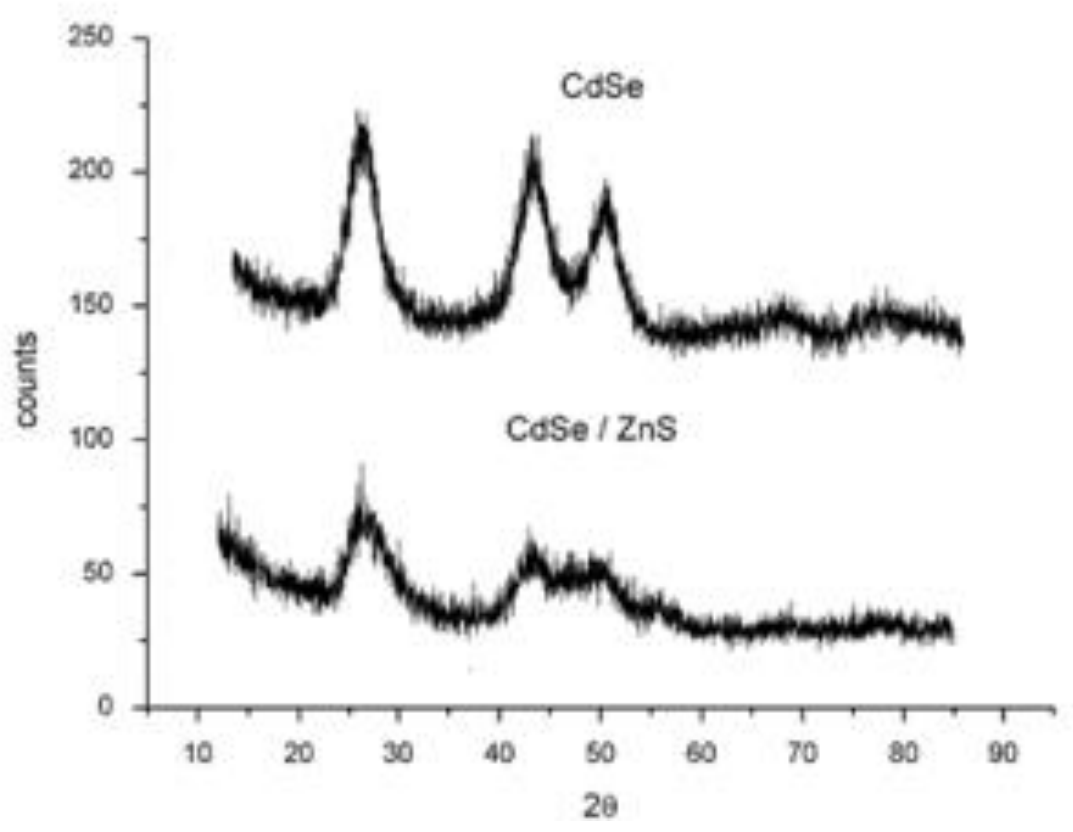

Figure 2.15. XRD profile is softened after shelling, suggesting that the ZnS addition successfully modified the original structure. ${ }^{[12]}$

To further characterize the structure, Figure 2.16 is an FTIR measurement of the CdSe/ZnS core-shell quantum dots after anchoring and attaching the 2-MPA capping agent. In this figure, it is observed that there is a significant carbonyl terminating peak at $1710 \mathrm{~cm}^{-1}$, concluding that the surface of the $\mathrm{CdSe} / \mathrm{ZnS}$ core shells quantum dots are terminated with a carboxyl group on the surface. This carboxyl group is the area that will react with the heavy metal ions and result in the fluorescence quenching effect. The washing by ethanol is a purification step, and the focus of this image should be on the carbonyl FTIR peak. 


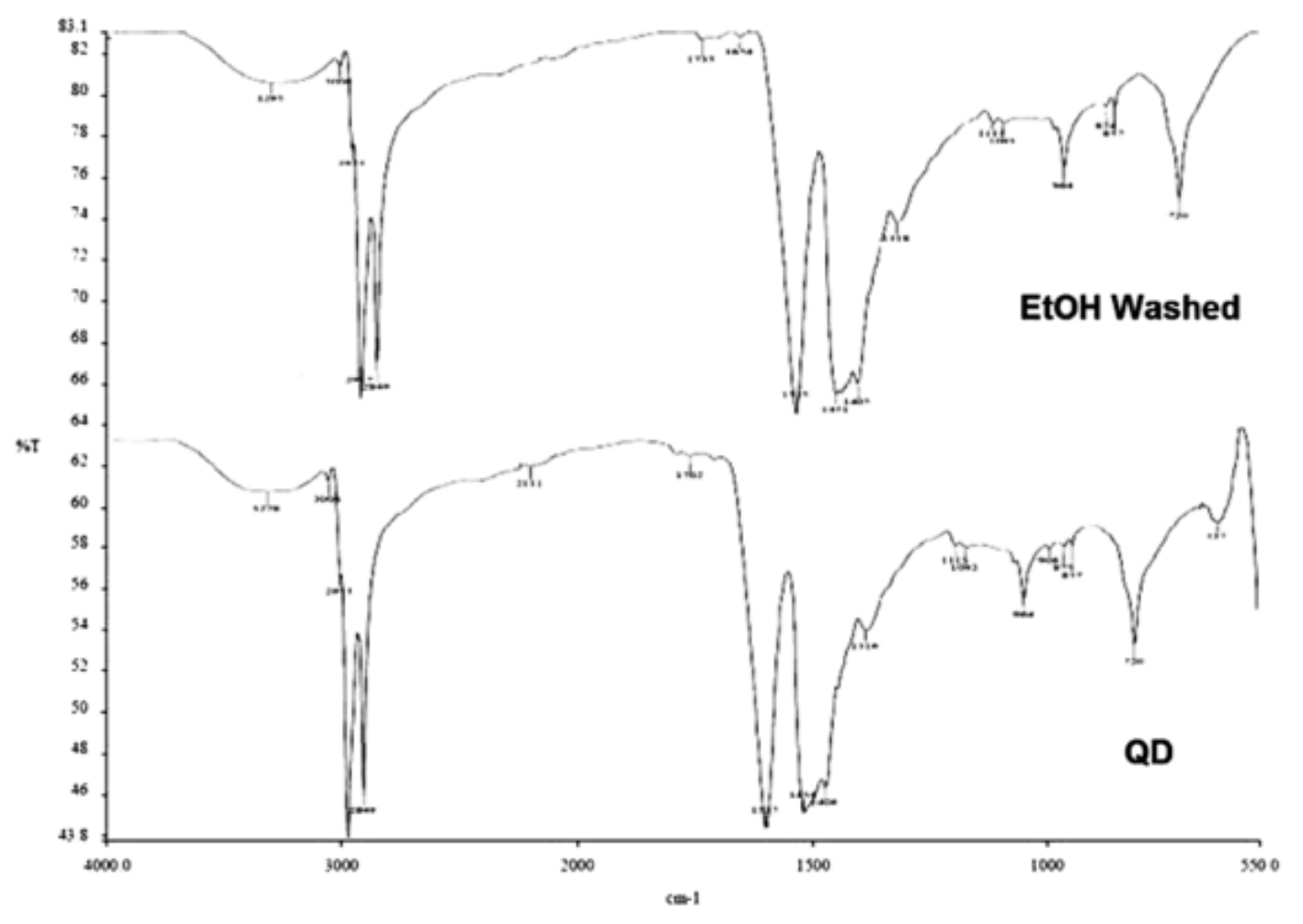

Figure 2.16. FTIR spectrum of the core-shell quantum dots indicates a strong peak at $1710 \mathrm{~cm}^{-1}$, suggesting that the 2-MPA capping agent is carbonyl terminated on the surface. ${ }^{[12]}$

The results of fluorescence quenching in the presence of heavy metal ions such as mercury, cadmium, and lead are provided in Figures 2.17-2.19, respectively. As expected, increasing the concentration of the heavy metal analyte results in the fluorescence being quenched - however, this response is different amongst the ions, suggesting that certain ions are more effective at reacting with the carbonyl terminal groups of the 2MPA. 


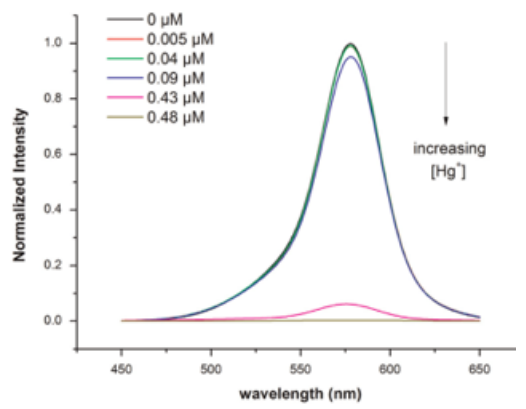

Figure 2.17. Fluorescence quenching observed for mercury ions. ${ }^{[12]}$

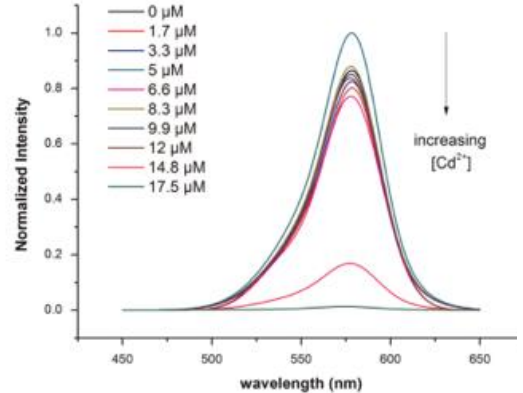

Figure 2.18. Fluorescence quenching observed for cadmium ions. ${ }^{[12]}$

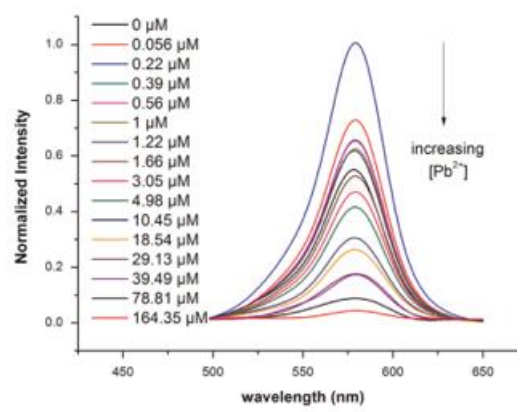

Figure 2.19. Fluorescence quenching observed for lead ions. ${ }^{[12]}$

For example, comparing the fluorescence quenching results between mercury and lead brings forward sensitivity concerns for the respective ions. Lead is considerably more sensitive, and the stepwise fluorescence quenching occurs relatively linearly as the concentration of the lead ion increases in comparison to the mercury ions. A graph of the normalized intensity against the analyte concentration is shown below in Figure 2.20, verifying the linear behavior (over a logarithmic scale) observed in one ion system over the other. 


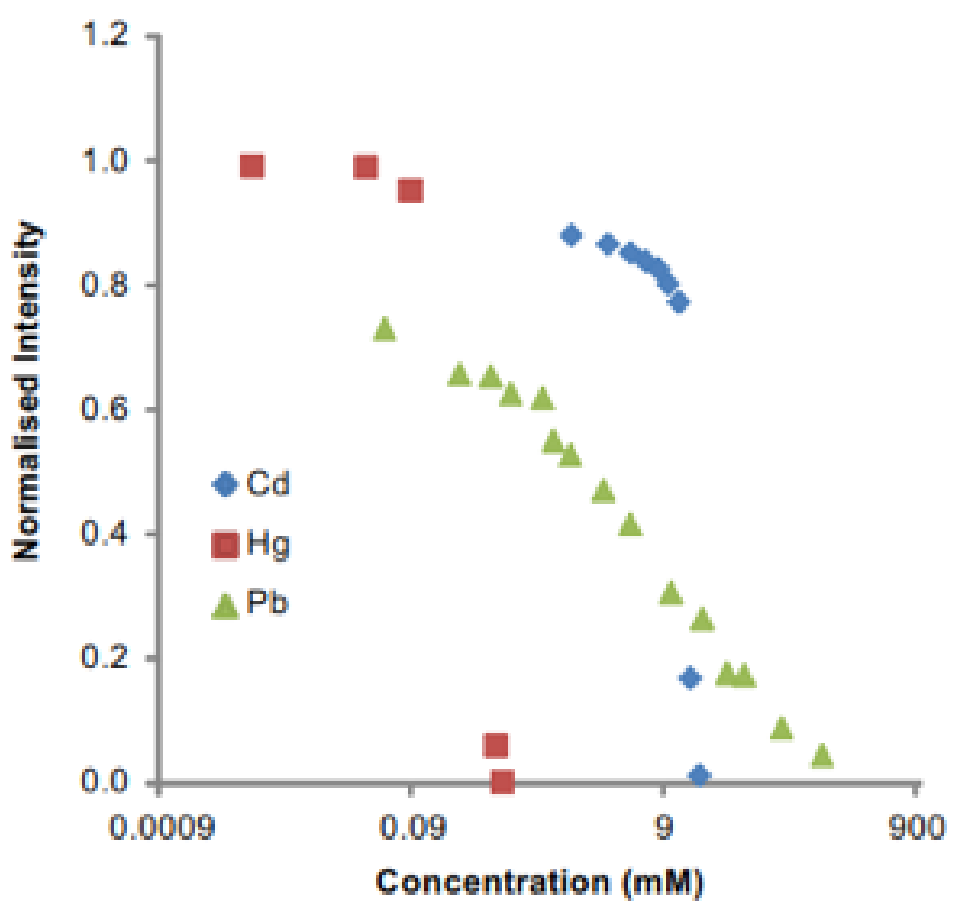

Figure 2.20. Intensity behavior as concentration increases differs amongst the three ions, suggesting different mechanisms for interacting with the capping agent and fluorescence quenching. ${ }^{[12]}$

This previously reported research demonstrates that heavy metal ions are capable of being sensed at nanomolar concentrations by fluorescence quenching of $\mathrm{CdSe} / \mathrm{ZnS}$ core-shell quantum dots. Quenching behavior is different amongst the three ions, suggesting that all three interact differently in reacting with the 2-MPA capping agent.

Now that heavy metal ions can be detected, one major obstacle preventing the success of these materials is their limited long-term stability. In this research, the 2-MPA capped quantum dots were deposited into a polyurethane primer coating. As shown in Figure 2.21, the fluorescence intensity of the quantum dots decreases greatly with age - the left peak representing the coating naturally decaying with age and the right peak the quantum dot performance decreasing as well. There are several viable and accessible strategies to strengthen the quantum yield and increase the lifetime of the quantum dot, however these come at the cost of increasing the complexity of the synthesis. This image presents the major conundrum with using these 
materials for sensing purposes: there is a sensitive balance and struggle between optimizing for performance versus lifetime.

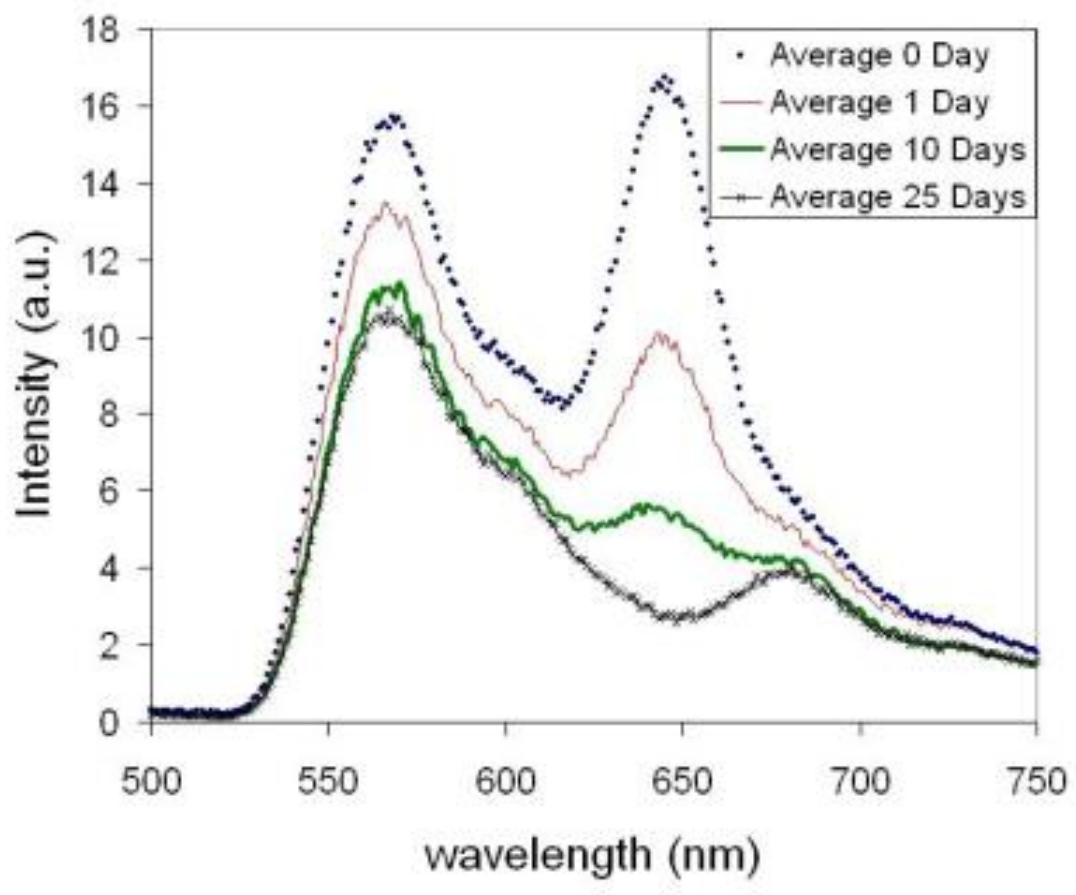

Figure 2.21. Fluorescence intensity decreases in both the (left) coating and the (right) quantum dot over time - this brings up the ultimate challenge with quantum dots and that is their limited lifetime, a weakness that must be addressed and optimized for sensing to be effective long-term. ${ }^{[12]}$

\subsection{Transition for Use in Sensing for Corrosion Events}

Several industrial and commercial segments of the economy are subject to risk from the potential damage that corrosion events can bring forward. Equipment damage, such as the case when a carbon steel pipeline is corroded in the presence of water and carbon dioxide, is the leading failure mechanism lending way to an estimated $\$ 276$ billion annually in damage for the United States alone ${ }^{[13]}$. Translating this figure into GDP leads to a staggering $3.1 \%$ damage due to corrosion failure ${ }^{[13]}$. Therefore, the purpose of this research is to build upon current sensing metal ion research to using these quantum dots for detecting corrosion and 
other aging related mechanisms and the ions they release during these aging mechanisms ${ }^{[13]}$. This sensing can also be extended for an in-situ approach for detecting changes in aging coating mechanisms as they occur or as controlled exposure doses are applied. Industries such as shipbuilding and seawater desalinations readily interact with saline water and other harsh environmental conditions that promote corrosion ${ }^{[13]}$. When two metals and alloys have different electric potentials, galvanic corrosion will occur in the presence of an electrolyte. Such is the case for industries where several metal and alloy systems are used such as within the electronics industries or with high power throughput systems such as power plants. 


\section{$\underline{3 \text { Review of Corrosion and Its Redox Reactions }}$}

A common participant in corrosion reactions is metallic iron, which readily reacts with water, hydrogen, and oxygen readily to initiate the corrosion process. Despite the formation of iron oxides when reacting with oxygen, corrosion itself can only be slowed down and not eliminated from occurring. The heterogeneity of multiple ions within alloy systems promotes the formation of anodes and cathodes due to each ion having different electrical potentials, as shown in Figure 3.1 for an iron carbon system ${ }^{[14]}$. Often corrosion is a phenomenon that assumes a preventative perspective rather than the luxury of completely removing it from the equation.

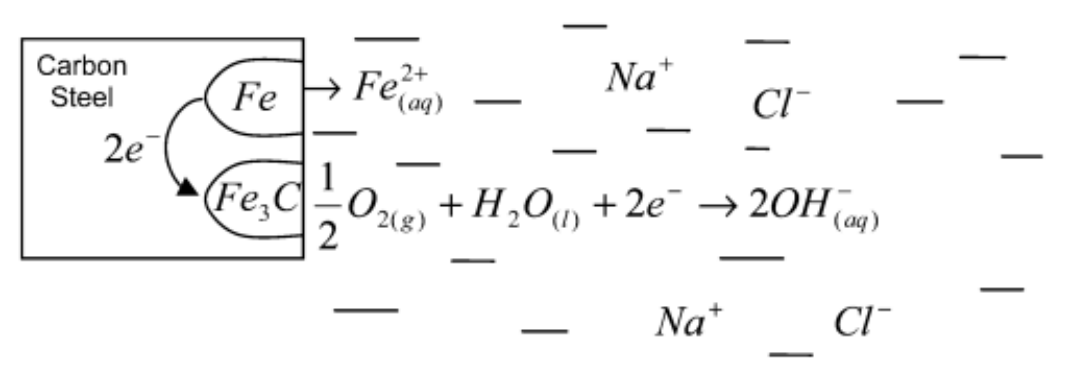

Figure 3.1. Electrochemical corrosion due to formation of a cathode at $\mathrm{Fe}_{3} \mathrm{C}$ and an anode at $\mathrm{Fe} .{ }^{[14]}$

A summary of the corrosion reactions for iron in a water system is summarized below ${ }^{[14]}$. Rusting is a form of corrosion that is an electrochemical process that requires the presence of water, oxygen, and an electrolyte. (i) The process begins with the oxidation of iron to form aqueous iron as well as two free electrons. (ii) These electrons are immediately consumed by the hydrogen ions present in the water or oxygen to produce more water. (iii) If the $\mathrm{pH}$ in the system is low, the hydrogen atoms will readily consume the electrons to form hydrogen gas. (iv) The hydroxide ions will react with the iron ions from the initial oxidation step to form iron hydroxides that appear as green rust. (v) The remaining iron(III) ions can also react with hydrogen and oxygen to form iron(III) oxides as shown in (vi). 
(i) $\mathrm{Fe}(s) \rightarrow \mathrm{Fe}^{2+}(a q)+2 e^{-}$

(ii) $4 e^{-}+4 H^{+}(a q)+O_{2}(a q) \rightarrow 2 \mathrm{H}_{2} \mathrm{O}(l)$

(iii) $2 H^{+}(a q)+2 e^{-} \rightarrow H_{2}(g)$

(iv) $\mathrm{Fe}^{2+}(\mathrm{aq})+2 \mathrm{OH}^{-}(\mathrm{aq}) \rightarrow \mathrm{Fe}(\mathrm{OH})_{2}(\mathrm{~s})$

(v) $4 \mathrm{Fe}^{2+}(a q)+4 \mathrm{H}^{+}(a q)+\mathrm{O}_{2}(a q) \rightarrow 4 \mathrm{Fe}^{3+}(a q)+2 \mathrm{H}_{2} \mathrm{O}(\mathrm{l})$

(vi) $\mathrm{Fe}^{3+}(\mathrm{aq})+3 \mathrm{OH}^{-}(\mathrm{aq}) \rightarrow \mathrm{Fe}(\mathrm{OH})_{3}(\mathrm{~s})$

The purpose of the following sections is to introduce current and traditional mechanisms that have historically been used to detect or prevent corrosion from occurring in paint and coatings systems. These topics were briefly researched and is a viable direction for the future of this research to further improve the sensing potential of the technology.

\section{$\underline{\text { 3.1 Performance Additives in Organic Coatings Systems }}$}

Paints and coatings are ideal candidates for modification by performance additives to protect the substrate it covers from the surrounding environment. Traditional organic coatings have utilized this in the form of improving barrier properties with the addition of fillers and performance additives that slow down ionic movement that leads to corrosion ${ }^{[15]}$. The issue is that the presence of these fillers and additives may negatively affect the performance of the coating. For example, if these additives disrupt the rheology of the coating, it would therefore influence and change how it is deposited onto the substrate. The most common diagnosis for substrate failure despite having these protective additives is due to intrinsic defects or damage that is accumulated in service or from surface contamination.

Taking into consideration defects in deposition and the lack of homogeneity in having a perfect coating, there is possibility in a risk that corrosion can occur in any region where these defects are present. For example, if there is a small hole in the surface of the coating, ions can utilize that region and travel through to the substrate promoting corrosion. Conversely, if the coating is deposited such that there is an air void at 
the interface between the coating and substrate, this also can act as a region where ions can become trapped and travel to promote the corrosion redox reactions ${ }^{[14]}$. An example of this phenomena is provided in Figure 3.2 .

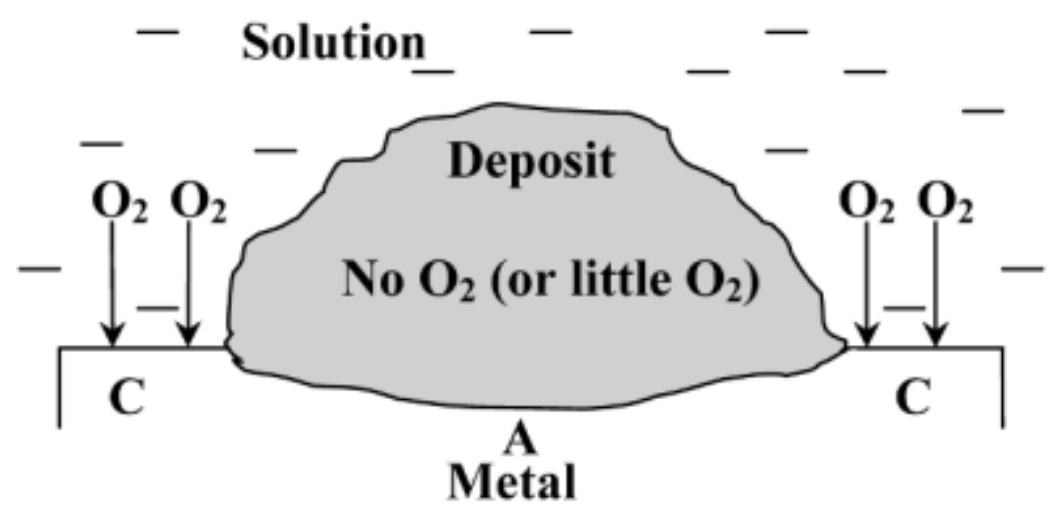

Figure 3.2. Example schematic of corrosion driven by defects caused upon by the presence of an air void from incorrect deposition. ${ }^{[14]}$

\subsection{Improving Barrier Properties}

Organic coatings also provide a benefit to the substrate in that they can improve the barrier properties by protecting the substrate from the surrounding environment ${ }^{[15]}$. Like the importance in properly depositing the coating over the substrate, barrier properties are only improved if deposition is performed correctly. By having an additional layer between the substrate and the environment, the coating acts as a physical barrier to moisture ${ }^{[15]}$. Polymer blends are typically used for producing barrier materials since the expensive barrier polymers such as aromatic polyester or poly(amino ethers) are a small amount in comparison to the inexpensive matrix material. Blends are also considerably less complicated to manufacture and process in comparison to other manufacturing techniques such as copolymer extrusion. The effect on the barrier properties for blends is predominantly dependent upon the morphology of the nanoparticles comprising it as shown in Figure 3.3. 

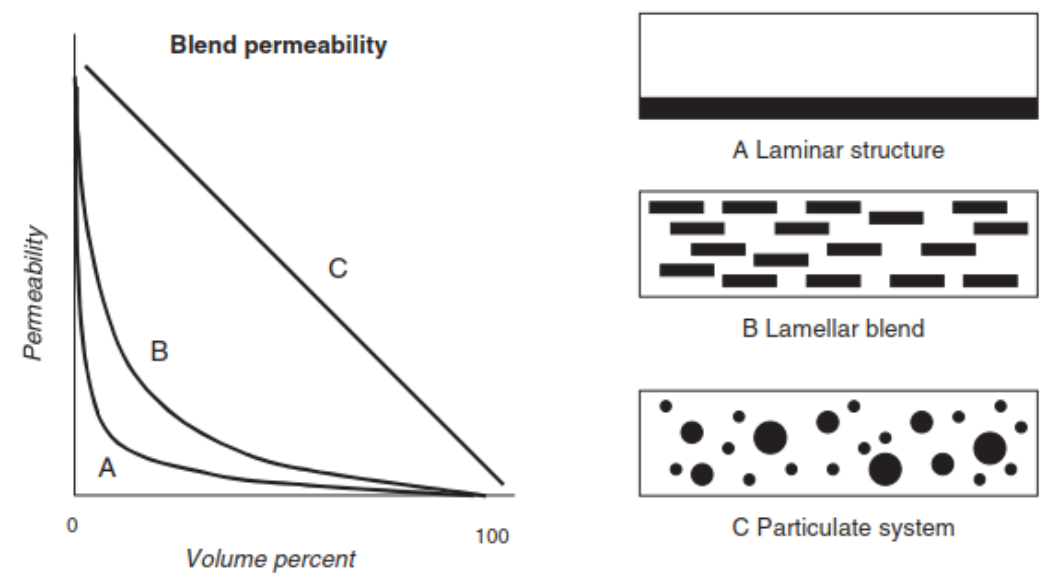

Figure 3.3. The morphology of the blend is important for describing the impact on barrier properties ${ }^{[15]}$.

Blends also can contain other additives present that can increase adhesion or improve manufacturability or processability of the coating ${ }^{[15]}$. Adding nanoparticles increases the tortuosity of the diffusion path. These nanoparticles must have a specific configuration or alignment or else they will promote vapor or oxygen permeability by providing additional transport channels as shown in Figure 3.3. In this example, the particulates in 3.3(c) are not densely packed, providing faster transport than the blend where the platelet additives are settled or nearly arranged near the substrate and coating interface in 3.3(a). The arrangement in 3.3(b) is a better example of how the alignment and structure of the nanoparticles can decrease permeability and thereby increase the barrier properties. However, again the same problem arises in that it is difficult to properly disperse these nanoparticles.

\section{$\underline{\text { 3.3 Surface Preparation for Better Coating Deposition }}$}

Considering the importance of performing the deposition correctly onto a substrate, the surface and substrate is often pretreated in advance, so the coating can adhere to it as successfully as possible ${ }^{[16]}$. This primarily involves cleaning the substrate properly to rid it of any surface contaminants that may interfere with the deposition process. Additionally, sometimes the surface is treated by increasing its roughness such as with a chemical or surface treatment to increase the adhesion of the coating by giving the coating more regions to adhere to. This process is large application specific, but there is always a surface or substrate 
preparation stage required to ensure that a coating will be successful in aiding towards optimal adhesion and deposition.

Surface roughening is one of the most prominent surface treatments used to aid in the enhancement of better adhesion, allowing for more interlocking between the coating and the substrate ${ }^{[16]}$. However, excessive roughening may inhibit the benefits of this technique, as shown in Figure 3.4. Here, it is observed that the coating does not fully penetrate and interlock with the surface features, resulting in incomplete adherence to the substrate. Since the coating does not completely penetrate the pore features and crevices, the actual interfacial contact area is less than the geometric area.

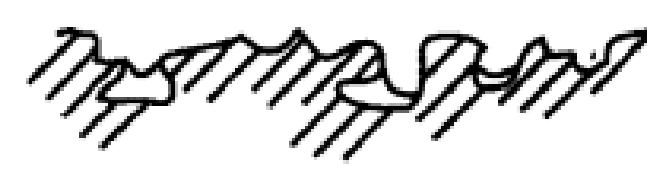

Figure 3.4. Schematic for incomplete penetration due to excessive surface roughening. ${ }^{[17]}$

The investigation of adhesion requires examination of both the surface of the substrate and its interactions with the coating. Metal substrates are often coated with oils that improve processability during the manufacturing stage. These oils are frequently wiped down with suitable solvents before the coating procedure is performed. In addition, some industries utilize vapor degreasing which condenses the cleaning solvent onto a hanging metal substrate ${ }^{[17]}$. The droplets that condense immediately dissolves the contaminant oils and eventually drips down into a collection container that is purified and recycled to repeat the process.

An alternative, yet more involved, approach is to treat the surface of the metal chemically through chemical pretreatments. Generally used are phosphate-based coatings that mildly etch the surface, greatly increasing the adhesion of the coating ${ }^{[17]}$. Other phosphates that can be utilized include zinc acid phosphates and its related family, such as the coprecipitate of zinc and ferric phosphates shown in Figure 3.5 below. 


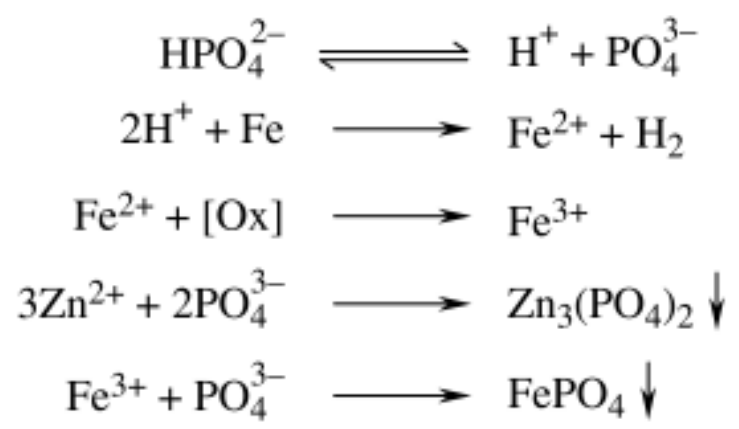

Figure 3.5. Series of reactions for producing the coprecipitate of zinc and ferric phosphates. ${ }^{[17]}$

For aluminum surfaces, there already is a native aluminum oxide present on the surface. This layer is rather dense however thin and sometimes is not sufficient alone for applications where the substrate will be subject to harsh environments. The most common treatments for aluminum substrates are those with chromates such as acid cycling through a combination bath containing chromate, fluorides, and ferricyanide salt ${ }^{[18]}$. This practice, however, has not had much popularity due to the presence of toxic cyanide and chromate components in the treatment.

In general, adhesion testing is performed through a standardized tape test. Alternative test methods include using a penknife to scrape the coating from the substrate ${ }^{[18]}$. Despite these tests being standardized, there are far too many adhesion parameters to consider so better techniques and processes must be developed to accurately gauge the adhesion strength of a coating after it is deposited.

\subsection{How Corrosion Inhibitors Operate}

Although the polymer itself is the first line of defense, corrosion inhibitors can supplement in preventing corrosion from occurring in the system. This can occur chemically, where inhibitor is readily adsorbed and chemically reacts with the substrate, often forming a thin film over the surface of the substrate due to its low surface energy ${ }^{[18]}$. This increases the electrical potential of the substrate, which ultimately leads to the metal natively passivating and readily forming its metal oxide film. In addition to acting as a physical 
barrier, the corrosion inhibitors also can interact with corrosive ions or components to shut them off and prevent them from causing corrosion by blocking its active sites ${ }^{[18]}$. There are a large and vast class of corrosion inhibitors - all with unique characteristics and mechanisms in preventing corrosive components from partaking in the redox reactions. Some of these are summarized in Figure 3.6.

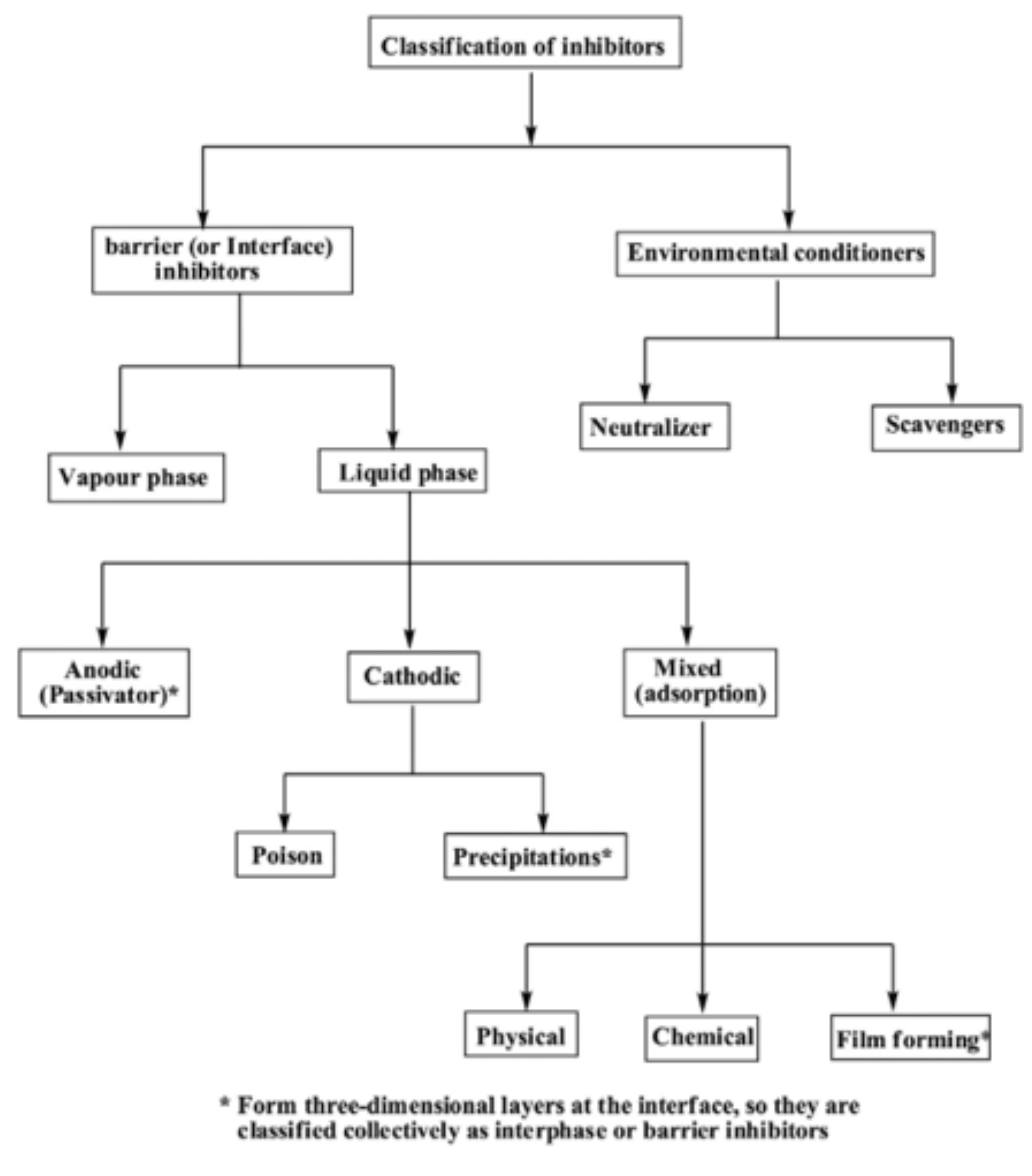

Figure 3.6. A diverse range of corrosion inhibitors can be used to increase a coating's performance. [18]

\subsection{The Many Types of Organic Corrosion Inhibitors}

For the specific application and technology this research is focused on, the classification to investigate is that of organic corrosion inhibitors that will be able to be deposited into organic coating systems such as architectural and other high-performance paint coatings. Organic coatings operate by blocking the anodic and cathodic sites, preventing any wear or erosion of the metal substrate due to eliminating the redox 
reactions entirely ${ }^{[18]}$. This class of inhibitors is very diverse with usages in acidic and alkaline environments as examples of its usage flexibility in different media. Reaction schemes are shown for two common organic inhibitors for use in acidic and alkaline environments below in Figure 3.7 and 3.8, respectively ${ }^{[18]}$. Corrosion inhibitors may also involve surfactants, which may be beneficial for specific coatings systems a nonionic example in polyaniline hydrogen chloride Figure 3.9 and a fatty amido-cationic in Figure 3.10 ${ }^{[18]}$. In addition, a large list of structures for synthetic organic corrosion inhibitors specifically for use in corrosive media is provided in Figure 3.11, demonstrating the gallery of possible candidates for organic coating systems.

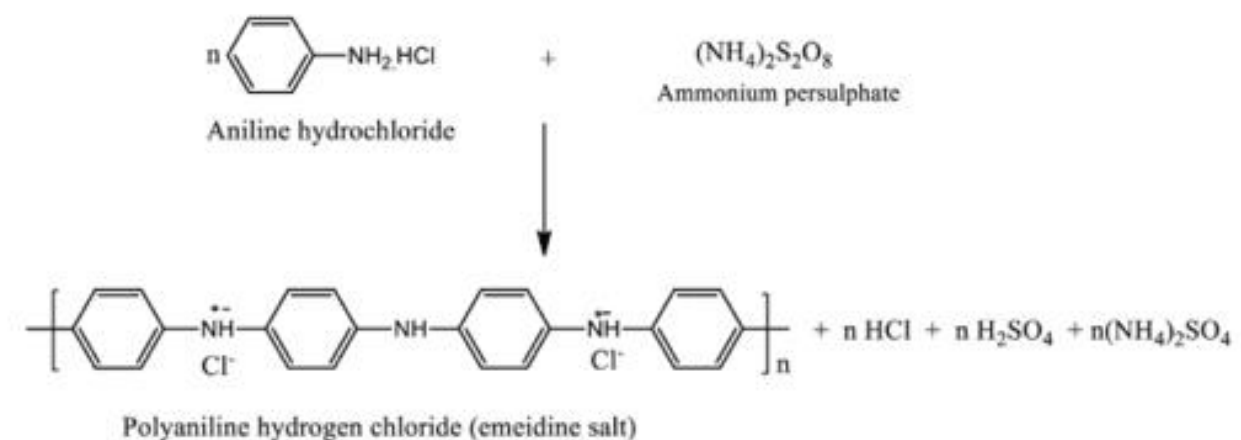

Figure 3.7. Example of a corrosion inhibitor suited for acidic environments. ${ }^{[18]}$ 

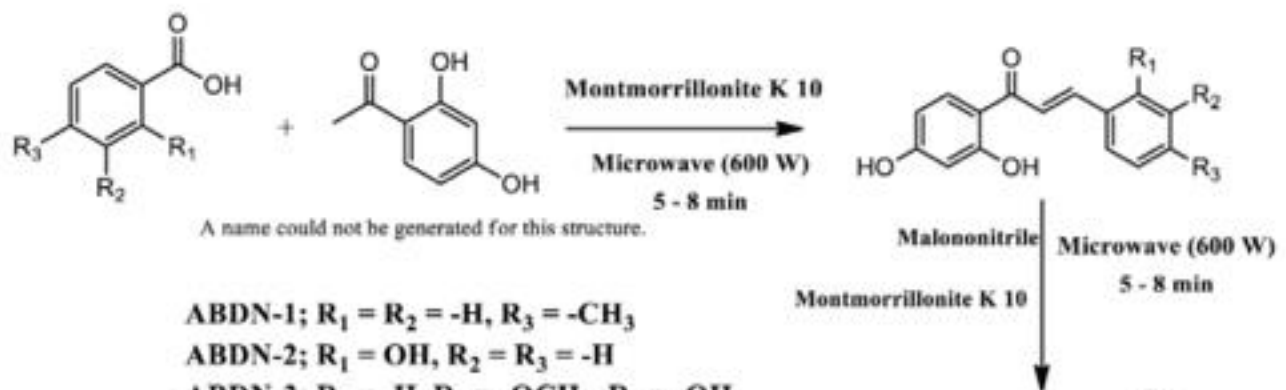

$A B D N-1 ; R_{1}=\mathbf{R}_{2}=-\mathbf{H}, \mathbf{R}_{3}=-\mathrm{CH}_{3}$ $A B D N-2 ; R_{1}=O H, R_{2}=R_{3}=-H$ $A B D N-3 ; R_{1}=-H, R_{2}=-\mathrm{OCH}_{3}, \mathrm{R}_{3}=-\mathrm{OH}$

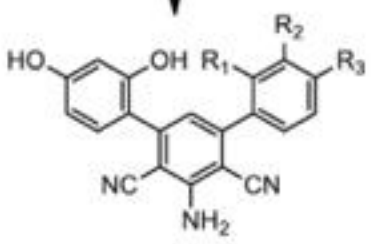

Figure 3.8. Example of a corrosion inhibitor suited for alkaline environments. ${ }^{[18]}$<smiles>Cc1ccc2[nH]nnc2c1</smiles>

Tolyltriazole

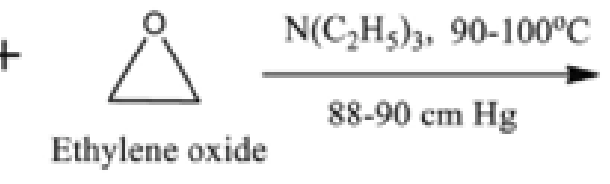

Ethylene oxide

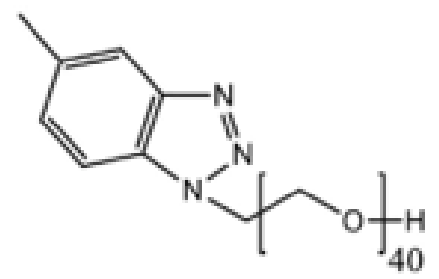

EMB inhibitor

Figure 3.9. Example of a nonionic surfactant corrosion inhibitor. ${ }^{[18]}$ 


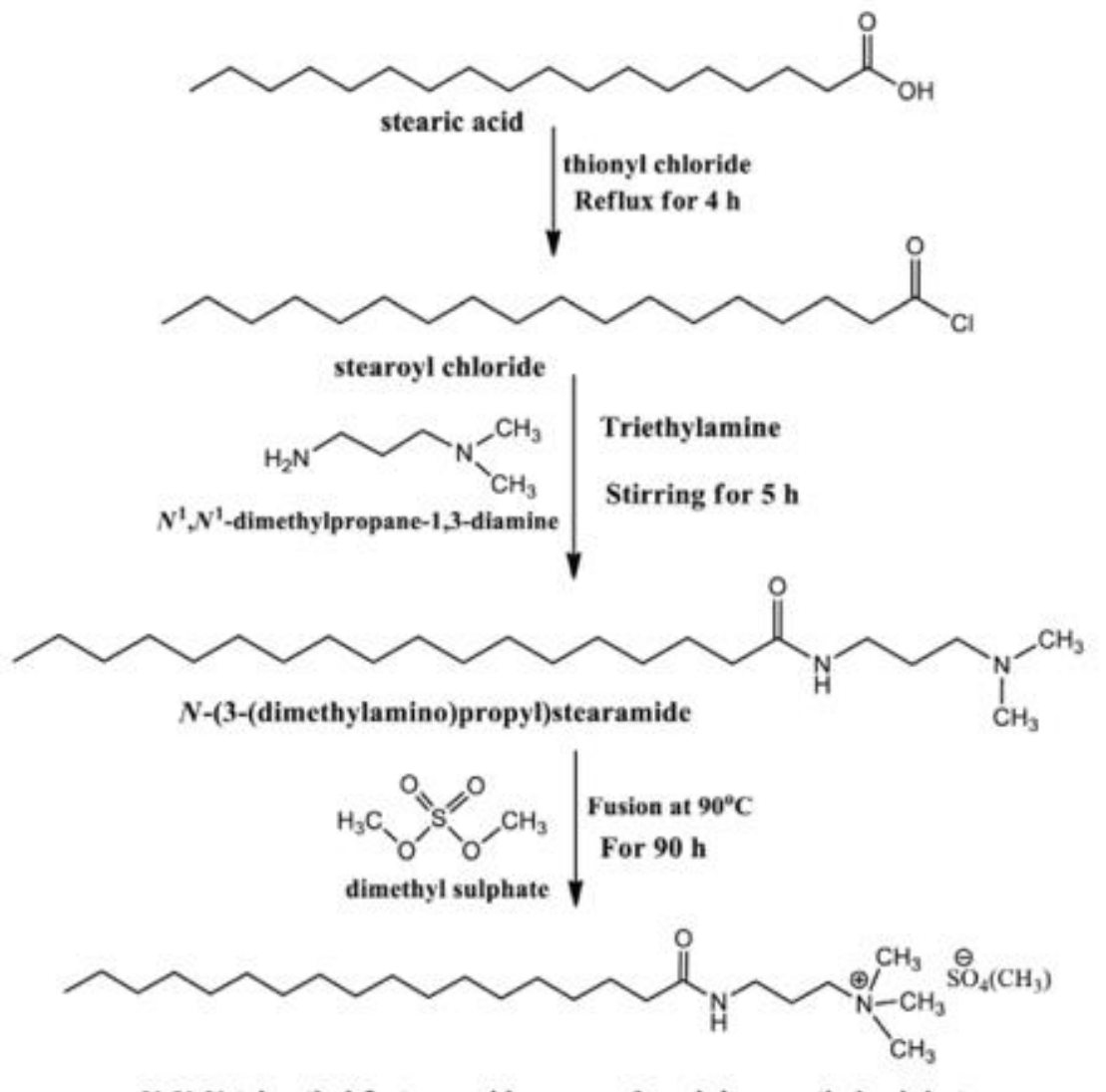

$\mathrm{N}, \mathrm{N}, \mathrm{N}$-trimethyl-3-stearamidopropan-1-aminium methyl sulphate

Figure 3.10. Example of a fatty amido-cationic surfactant corrosion inhibitor. ${ }^{[18]}$ 


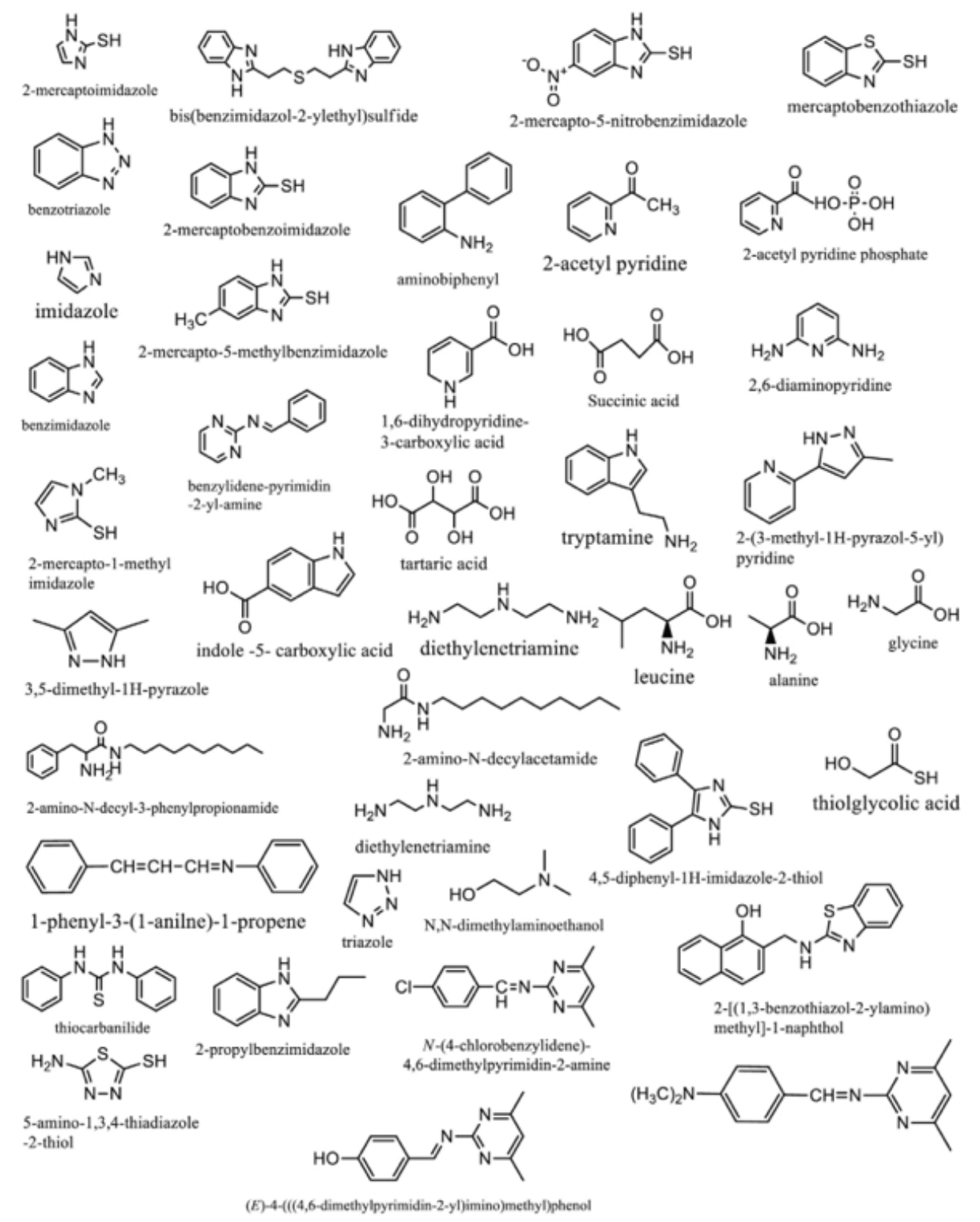

Figure 3.11. Examples of the large number of possibilities of different organic corrosion inhibitors. [18] 


\section{$\underline{\text { 3.6 Surface Modification for Increased Quantum Dot Passivity }}$}

The mechanism of shutting down active sites by corrosion inhibitors is closely related to that of surface passivation by surface capping agents. Surface modification can be utilized to greatly increase the photoluminescent properties of quantum dots. One example is poly(proionylethylene-imine-coethyleneimine) as another viable capping agent. Surface passivation can be explained by any processing technique that disables or inhibits the presence of active sites on the surface of the quantum dot system.

The inhibition of active sites protects the quantum dot system from interacting with its surrounding environment as well as increasing the quantum yield performance of the system since inhibiting the active sites discourages and prevents electron-hole pair recombination ${ }^{[1]}$. The most common form of surface passivation is through the attachment and anchoring of capping agents that chemically react with the active sites and inhibit them from external reactions by making the system more chemically inert.

Alternatively, surface passivation can also be achieved by modifying the surface such that it only is responsive to a selective stimulus. In this example, the quantum dot has enhanced reactivity to a specific analyte, but its increased specificity protects the quantum yield and its characteristic signal. Depending on the application, different functional groups can be attached as surface capping agents for this purpose. Some examples of common capping agents that serve this role include ligands such as thioglycerol, mercaptoacetate, cysteine, and glutathione ${ }^{[1]}$. Amines such as hexadecylamine and n-hexylamine can also be utilized ${ }^{[1]}$.

A visual example of the surface passivation effect is observed in Figure 3.12, where the brightness and intensity of the quantum dots are greatly increased upon irradiation with a $395 \mathrm{~nm}$ UV source. 

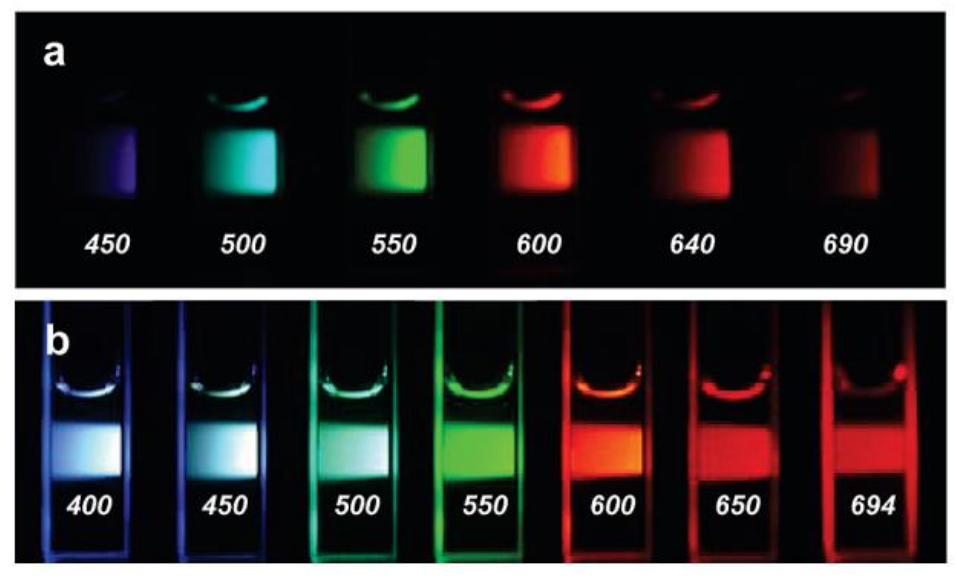

Figure 3.12. Improved quantum yield observed due to surface passivation improving the stability and durability of the quantum dot system. ${ }^{[19]}$

The addition of organic superacids as capping agents significantly increases the photoluminescence quantum yield of $\mathrm{MoS}_{2}$ quantum dots from $1 \%$ pre-treatment to $95 \%$ post-treatment ${ }^{[20]}$. One example of a superacid is the nonoxidizing bis(trifluoromethane) sulfominide TFSI. With its strong protonating ability, it removes $\mathrm{MoS}_{2}$ contaminants and results in passivation of sulfur vacancies by rearrangement of sulfur adatoms. The passivation behavior can be replicated in crystalline silicon surfaces by deactivating recombination centers or saturating free silicon bonds, thus preventing them from reacting with the external environment ${ }^{[20]}$.

In this study, the researchers synthesized $\mathrm{CuInS}_{2} / \mathrm{ZnS}$ core-shell quantum dots in toluene and capped with bis(trifluoromethane) sulfominide. The $\mathrm{CuInS}_{2} / \mathrm{ZnS}$ quantum dots were left to soak and react with bis(trifluoromethane) sulfominide at different molar ratios and at different temperatures. The results of both the photoluminescence and absorbance measurements are shown in Figure 3.13 below. 

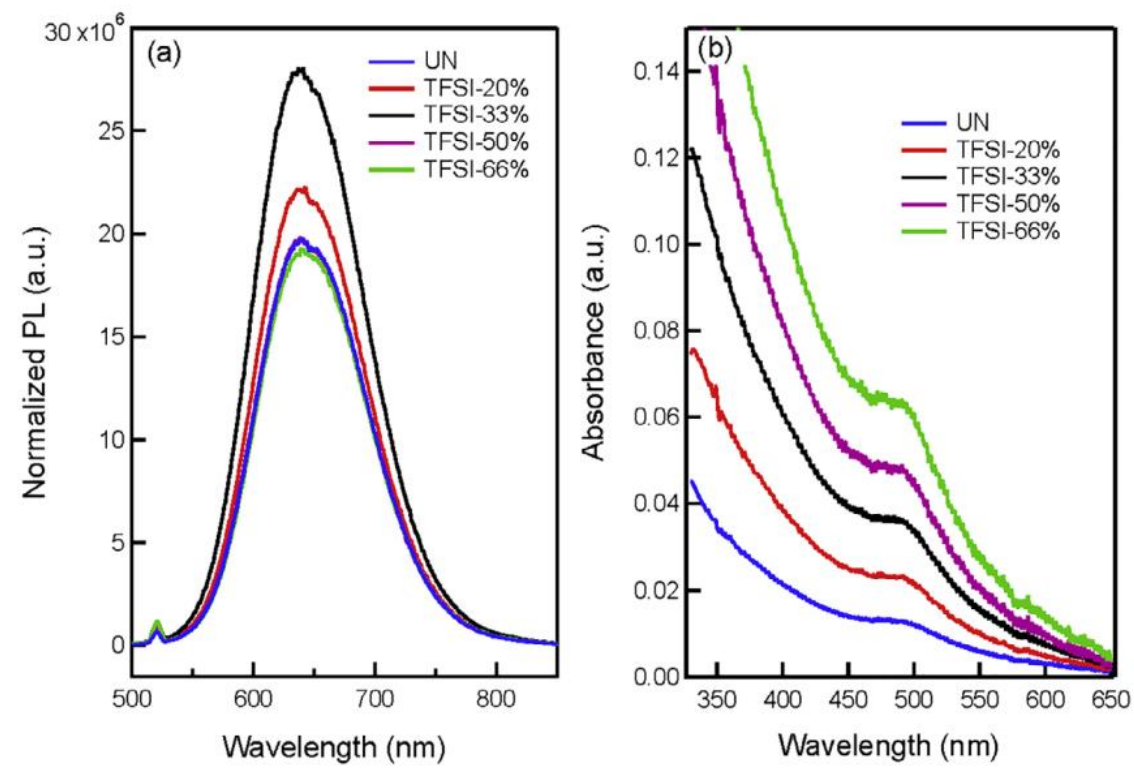

Figure 3.13. Addition of TFSI increases quantum yield and optical performance as shown in (a) fluorescence and (b) UV-Vis measurements. ${ }^{[12]}$

From the peaks, it is observed that the normalized intensity and absorbance values increase significantly even with the smallest contribution of TFSI, verifying that the organic superacid capping agent aids in surface passivation. Surface modification by saturating the surface vacancies can also be observed by looking at the particle size distribution. Using TEM, Figure 3.14 was generated. The effect of TFSI in eliminating active sites on the surface is demonstrated by the distribution becoming narrower. 

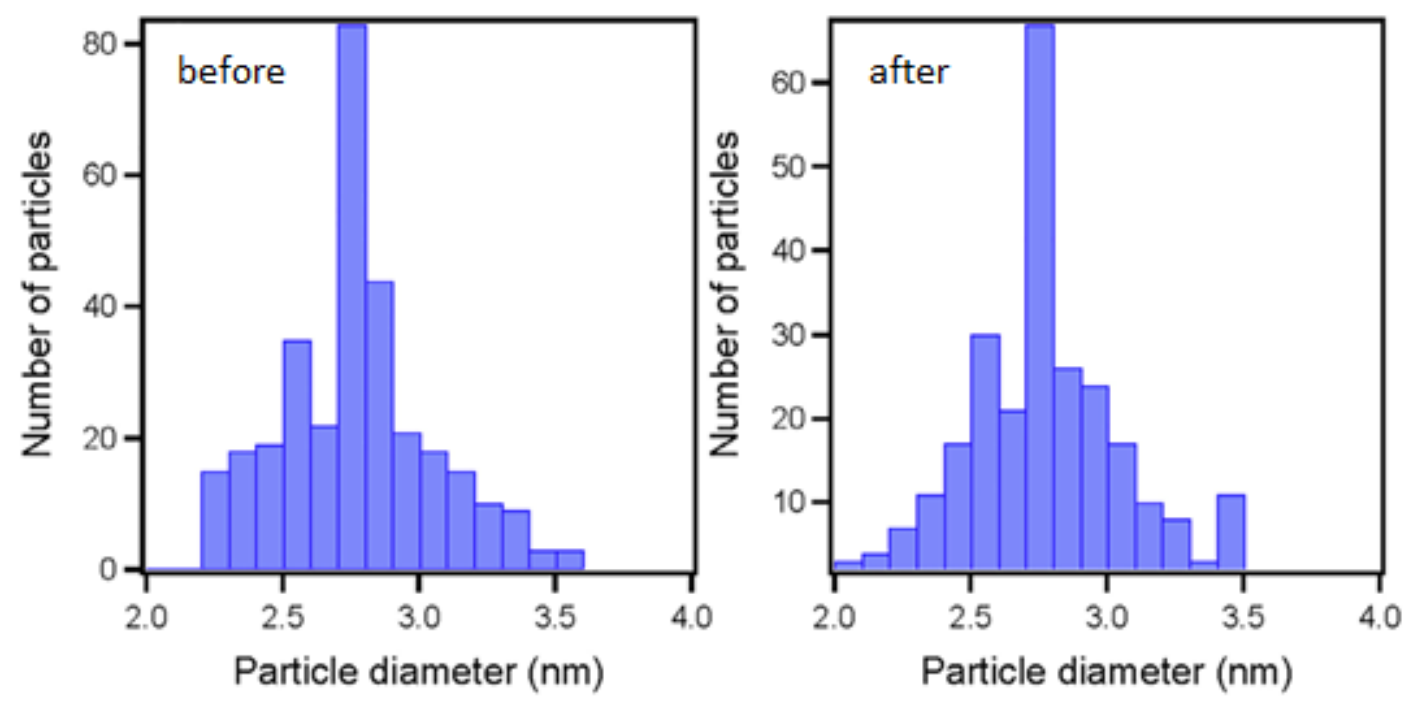

Figure 3.14. Particle size distribution is more monodisperse after the surface passivation by TFSI. ${ }^{[12]}$

The above measurements can also be supplemented through the usage of HRTEM and Raman techniques. HRTEM can provide meaningful images describing the crystallinity and lattice spacings after surface modification - the structure should not change since the capping agent is only aiding in surface passivation rather than reacting with the surface and changing the composition of the quantum dot structure. Raman can also be utilized to compare the peak ratios at specific wavelengths to see if the crystal structure integrity is also maintained.

Surface passivation is a crucial strategy that must be explored to optimize the quantum performance of any quantum dot structure. Surface passivation shuts down the active sites on the surface of quantum dots, further stabilizing them and improving their performance in terms of narrowing the energy bands, the midgap states, and stability ${ }^{[21]}$. The surface is modified by ligand exchanges to stabilize the exterior of the quantum dots by substituting the long oleate ligands with shorter compounds that are not reactive with the surrounding environment.

One strategy was investigated by Zherebetskyy et al. who stabilized the surface of lead sulfate quantum dots by grafting hydroxyl ligands onto the oleic acids ${ }^{[21]}$. This modification stabilizes the system by maintaining net charge neutrality and thereby minimizes the surface energy of the system. Most ligand 
exchanges focus on removing the surface oleate components, so this strategy focuses on chemically modifying the surface by addition rather than subtraction. Cao et al. supported this strategy and focused their research on the suppression of hydroxyl ligands that grow during the synthesis of lead sulfide quantum dots ${ }^{[21]}$.

A compound that is often added for size tunability of quantum dots during growth is trioctylphosphine. This organic ligand reacts with the surface of a sulfide-based quantum dot and inhibits the active sites ${ }^{[22]}$. This results in higher quantum yield since the structure will be increasingly stabilized by the addition of trioctylphosphine as a surface capping agent. The side by side comparison UV-Vis measurements in Figure 3.15 demonstrate a greater absorbance measurement for the system with trioctylphosphine.
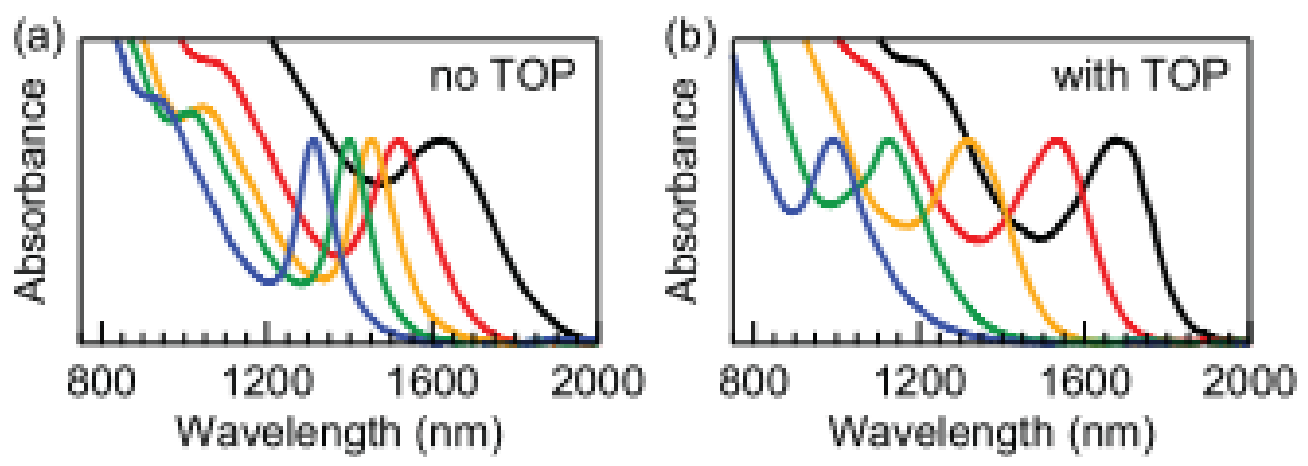

Figure 3.15. UV-Vis measurements have greater absorbance due to the increased stability provided by the addition of trioctylphosphine where (a) is without trioctylphoshine and $(b)$ is with the addition of trioctylphosphine. ${ }^{[22]}$

One disadvantage to surface passivation is that the presence of an additional shell layer outside the core may suffer from lattice mismatch ${ }^{[23]}$. Instead of protecting and stabilizing the fluorescence emitted by the core, the shell instead will effectively generate a new sub band gap state due to the lattice strain generated by the mismatch. This presents a new research topic to be investigated in the future if different shells instead of zinc sulfide are used to coat the cadmium selenide cores. Compatibility between cores, shells, and surface 
capping agents must be explored in greater detail to ensure that quantum yield is maximized, and surface passivation theories are preserved.

All these techniques should be utilized in future iterations of this research to further characterize the synthesized core-shell quantum dot structure. 


\section{Major Goals and Objectives}

The purpose of this research is to combine the fluorescence quenching behavior and response of core-shell quantum dot materials in the presence of heavy metal ions - particularly copper ions - as a sensor for corrosion events by detecting the presence of copper ions released as the material corrodes. This research primarily focuses on the synthesis of $\mathrm{CdSe} / \mathrm{ZnS}$ core-shell quantum dots and their characterization and early attempts at integrating them into polymer coating systems. The foundation of this research is centered around quantum dot synthesis, so future directions involve performing more research to successfully integrate functionality in depositing the quantum dots into different coatings systems. The major reaction that this research tackles is that of corrosion, summarized in Figure 4.1 below.

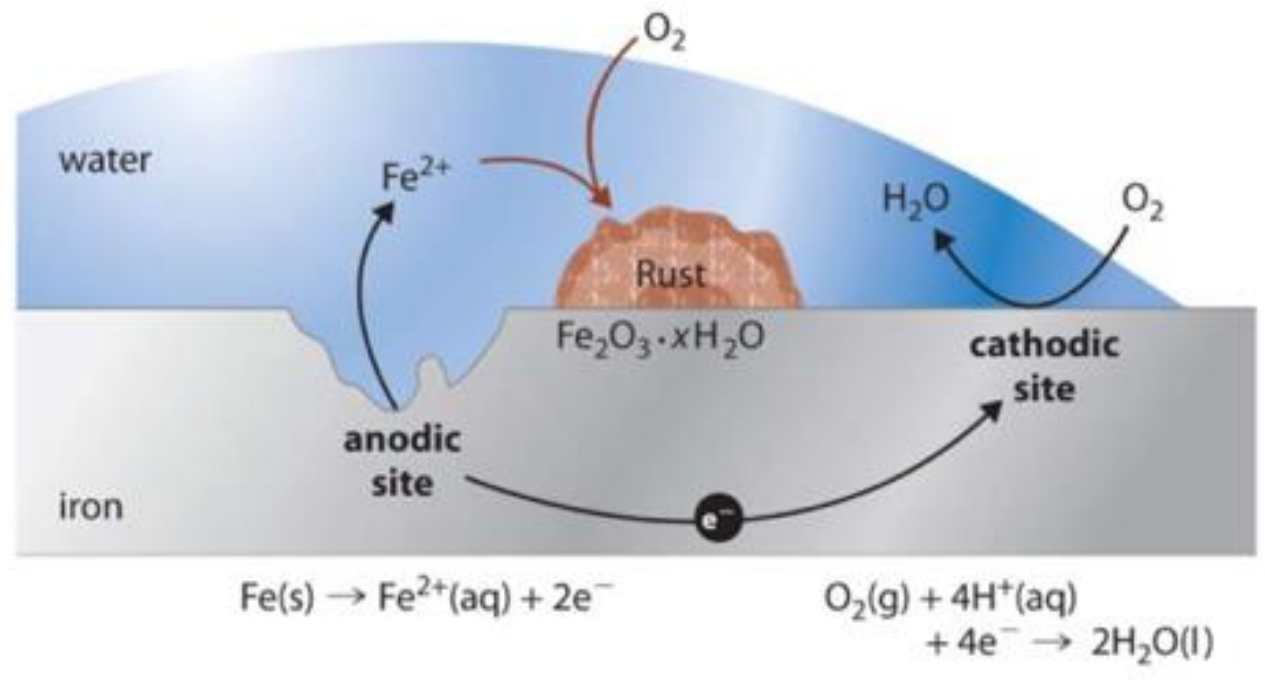

Figure 4.1. General summary for the corrosion redox reactions for an iron/water system. ${ }^{[24]}$

This research begins with outlining the specific steps necessary to construct and synthesize the key coreshell quantum dots - namely CdSe/ZnS core-shell quantum dots. Firstly, the cadmium selenide CdSe cores will be synthesized before performing optical characterization. From this, an accurate estimation of the core diameter is determined, and using those values, the proper amounts of the zinc and sulfide components for 
the zinc sulfide shells will be estimated. Using these values, the cores are then shelled to finally make the $\mathrm{CdSe} / \mathrm{ZnS}$ quantum dot systems.

These quantum dots were then deposited into a series of polymer and commercial paint coatings systems at different loadings. Preliminary images were taken to demonstrate the changes in fluorescence exhibited before and after the quantum dots were integrated into the coatings systems. 


\section{$\underline{5 \text { Materials and Equipment }}$}

Synthesis of the core-shell quantum dot systems requires suitable glassware capable for performing the synthesis, most importantly being three-neck round bottom flasks and condensers to attach to a Schlenk line. The reaction itself is not necessarily air sensitive, though the quality in quantum yield and optical performance is greatly increased when working in an air-sensitive environment. The condensers themselves are used for protective purposes in preventing the bumping and loss of any reagents or mixtures into the vacuum pump and Schlenk lines. Heating mantles were operated within the range of $20-260^{\circ} \mathrm{C}$.

Keep in mind that the working reagents and products from these experiments are nanoparticles, so careful consideration should be taken when handling these materials. Please also be courteous and discard of the nanoparticle waste accordingly. In addition to chemical safety, consider the use of proper safety and protective personal equipment. Hot injection requires the use of needles so a glass waste container should also be present for proper discarding of waste materials.

All chemicals were purchased from Sigma Aldrich, and all chemical grades were either technical or analytical grade and stored under a fume hood or in the appropriate safety cabinet. A list of all chemicals and important properties relevant to this study is provided in Table 5.1.

Optical characterization for the core-shell CdSe/ZnS quantum dot structures was obtained through UV-Vis spectroscopy and fluorescence spectroscopy. UV-Vis spectroscopy was performed on a Cary UV-Vis-NIR 5G spectrophotometer with measurements over the 350-800 nm range. Fluorescence spectroscopy was performed by a Cary Eclipse fluorescence spectrophotometer at $400 \mathrm{~nm}$ excitation wavelength over the 450-650 $\mathrm{nm}$ range. 
Table 5.1. Molar Masses and Densities of Synthesis Chemicals

\begin{tabular}{|c|c|c|c|c|}
\hline $\begin{array}{c}\text { Product } \\
\text { Number } \\
\text { (Sigma } \\
\text { Aldrich) }\end{array}$ & Chemical Name & Chemical Formula & Molar Mass & Density \\
\hline $202894^{[25]}$ & Cadmium oxide & $\mathrm{CdO}$ & $128.41 \mathrm{~g} / \mathrm{mol}$ & $\begin{array}{c}8.15 \mathrm{~g} / \mathrm{mL} \\
\text { at } 25^{\circ} \mathrm{C}\end{array}$ \\
\hline $229865^{[26]}$ & Selenium powder & $\mathrm{Se}$ & $78.96 \mathrm{~g} / \mathrm{mol}$ & $\begin{array}{c}4.819 \\
\mathrm{~g} / \mathrm{cm}^{3} \text { at } \\
20^{\circ} \mathrm{C}\end{array}$ \\
\hline $96479^{[27]}$ & Zinc oxide & $\mathrm{ZnO}$ & $81.39 \mathrm{~g} / \mathrm{mol}$ & $\begin{array}{c}5.61 \mathrm{~g} / \mathrm{cm}^{3} \\
\text { at } 20^{\circ} \mathrm{C}\end{array}$ \\
\hline $213292^{[28]}$ & Sulfur powder & $\mathrm{S}$ & $32.07 \mathrm{~g} / \mathrm{mol}$ & $\begin{array}{c}2 \mathrm{~g} / \mathrm{cm}^{3} \text { at } \\
20^{\circ} \mathrm{C}\end{array}$ \\
\hline $117854^{[29]}$ & Trioctylphosphine & $\mathrm{CH}_{3}\left(\mathrm{CH}_{2}\right)_{6} \mathrm{CH}_{2}-\mathrm{P}-\left[\mathrm{CH}_{2}\left(\mathrm{CH}_{2}\right) 6 \mathrm{CH}_{3}\right]$ & $370.64 \mathrm{~g} / \mathrm{mol}$ & $\begin{array}{c}0.831 \\
\mathrm{~g} / \mathrm{mL} \text { at } \\
25^{\circ} \mathrm{C}\end{array}$ \\
\hline $\begin{array}{c}364525 \\
{[30]}\end{array}$ & Oleic acid & $\mathrm{CH}_{3}\left(\mathrm{CH}_{2}\right)_{7} \mathrm{CH}=\mathrm{CH}\left(\mathrm{CH}_{2}\right) 7 \mathrm{COOH}$ & $272.46 \mathrm{~g} / \mathrm{mol}$ & $\begin{array}{c}0.895 \\
\mathrm{~g} / \mathrm{mL} \text { at } \\
25^{\circ} \mathrm{C}\end{array}$ \\
\hline $74738^{[31]}$ & 1-Octadecene & $\mathrm{CH}_{3}\left(\mathrm{CH}_{2}\right)_{15} \mathrm{CH}=\mathrm{CH}_{2}$ & $252.48 \mathrm{~g} / \mathrm{mol}$ & $\begin{array}{c}0.789 \\
\mathrm{~g} / \mathrm{mL} \text { at } \\
25^{\circ} \mathrm{C}\end{array}$ \\
\hline $07805^{[32]}$ & Oleylamine & $\mathrm{CH}_{3}\left(\mathrm{CH}_{2}\right)_{7} \mathrm{CH}=\mathrm{CH}\left(\mathrm{CH}_{2}\right)_{7} \mathrm{CH}_{2} \mathrm{NH}_{2}$ & $267.49 \mathrm{~g} / \mathrm{mol}$ & $\begin{array}{c}0.813 \\
\mathrm{~g} / \mathrm{mL} \text { at } \\
25^{\circ} \mathrm{C}\end{array}$ \\
\hline $\begin{array}{c}\text { CX1050 } \\
{[33]}\end{array}$ & Chloroform & $\mathrm{CHCl}_{3}$ & $119.38 \mathrm{~g} / \mathrm{mol}$ & $\begin{array}{c}1.48 \mathrm{~g} / \mathrm{mL} \\
\text { at } 25^{\circ} \mathrm{C}\end{array}$ \\
\hline$\underset{[34]}{\mathrm{AX} 0115}$ & Acetone & $\mathrm{CH}_{3} \mathrm{COCH}_{3}$ & $58.08 \mathrm{~g} / \mathrm{mol}$ & $\begin{array}{c}0.791 \\
\mathrm{~g} / \mathrm{mL} \text { at } \\
25^{\circ} \mathrm{C}\end{array}$ \\
\hline $459844^{[35]}$ & Ethanol & $\mathrm{CH}_{3} \mathrm{CH}_{2} \mathrm{OH}$ & $46.07 \mathrm{~g} / \mathrm{mol}$ & $\begin{array}{c}0.789 \\
\mathrm{~g} / \mathrm{mL} \text { at } \\
25^{\circ} \mathrm{C}\end{array}$ \\
\hline $34873^{[36]}$ & Heptane & $\mathrm{CH}_{3}\left(\mathrm{CH}_{2}\right)_{5} \mathrm{CH}_{3}$ & $100.20 \mathrm{~g} / \mathrm{mol}$ & $\begin{array}{c}0.684 \\
\mathrm{~g} / \mathrm{mL} \text { at } \\
25^{\circ} \mathrm{C}\end{array}$ \\
\hline
\end{tabular}




\section{$\underline{6 \text { Core Synthesis - Making the Cadmium Selenide Cores }}$}

Cadmium selenide quantum dot cores are synthesized by the following procedure involving the cadmium source and the selenium injection. A roadmap of the synthesis is provided in Figure 6.1 with the major steps outlined throughout the process.

\section{Prepare cadmium sources}

\section{Prepare selenium sources}

\section{Hot injection to synthesize CdSe cores}

\section{Quench reaction in water bath}

\section{Transfer to vial for purification step}

Figure 6.1. Synthesis roadmap for preparing the cadmium selenide quantum dot cores.

$0.6 \mathrm{mmol}$ of cadmium oxide, excess $3.4 \mathrm{~mL}$ of oleic acid, and $15 \mathrm{~mL}$ octadecene are added to a three-neck round bottom flask with stir bar. The resulting mixture is mixed thoroughly under ambient conditions for ten minutes before proceeding to degas the mixture at $110^{\circ} \mathrm{C}$ for 1 hour. The vacuum is then switched for nitrogen flow in addition to heating the mixture now to $220^{\circ}$ for 1 hour. Afterwards, the three-neck round bottom flask is lifted from the heating mantle and allowed to cool in ambient conditions for 15 minutes; simultaneously, the heating mantle is set to $110^{\circ} \mathrm{C}$. After cooling, the three-neck round bottom flask is lowered into the mantle and evacuated under vacuum for an additional 15 minutes. At this point, the vacuum 
is switched to nitrogen flow, and the flask is heated to $250^{\circ} \mathrm{C}$. The final mixture should be a clear paleyellow color.

$0.6 \mathrm{mmol}$ of selenium powder and $5 \mathrm{~mL}$ octadecene are added to a three-neck round bottom flask with stir bar. The resulting mixture is allowed to mix thoroughly under ambient conditions for ten minutes before proceeding to degas the mixture at $110^{\circ} \mathrm{C}$ for 1 hour. The vacuum is then switched for nitrogen flow in addition to heating the mixture now at $180^{\circ} \mathrm{C}$ for 1 hour. Once the mixture in the round bottom flask reaches this temperature, $0.6 \mathrm{~mL}$ trioctylphosphine is added. The addition of trioctylphosphine should gradually dissolve the selenium powder, resulting in a clear and transparent color.

A cold-water bath was prepared and placed on the workbench to be used for quenching the reaction. Then the selenium source is promptly and rapidly injected into the cadmium source with a large gauge needle to result in the preparation of the cadmium selenide cores. Reaction time will vary; however, a minimum of 45 seconds is recommended - the key here is to optimize the reaction time with the growth of the cores. Too much time and the cores will turn a dark black color, meaning that the synthesized cores are too big and no longer nanoparticles - in other words, the quantum dots will be "dead" and no longer fluorescent. The reaction flask is then left in the cold-water bath for at least 5 minutes before completion of the reaction.

The following table is an example synthesis for core-shell quantum dots. Table 6.1 is for the cadmium source and Table 6.2 the selenium source. Both tables below document the amounts of each compound added to synthesize a single batch of $\mathrm{CdSe} / \mathrm{ZnS}$ core-shell quantum dots.

Table 6.1. Example synthesis measurements for preparing the cadmium source.

\begin{tabular}{|c|c|c|}
\hline Compound Name & Amount Needed & Amount Added \\
\hline CdO powder & $0.1541 \mathrm{~g}$ & $0.1562 \mathrm{~g}$ \\
\hline Oleic acid & $6.086 \mathrm{~g}$ & $6.0961 \mathrm{~g}$ \\
\hline Octadecene & $30 \mathrm{~mL}$ & $\sim 30 \mathrm{~mL}$ by graduated cylinder \\
\hline
\end{tabular}


Table 6.2. Example synthesis measurements for preparing the selenium source.

\begin{tabular}{|c|c|c|}
\hline Compound Name & Amount Needed & Amount Added \\
\hline Se powder & $0.0948 \mathrm{~g}$ & $0.0992 \mathrm{~g}$ \\
\hline Octadecene & $10 \mathrm{~mL}$ & $\sim 10 \mathrm{~mL}$ by graduated cylinder \\
\hline Trioctylphosphine & $0.9972 \mathrm{~g}(1.2 \mathrm{~mL})$ & $1.2 \mathrm{~mL}$ by syringe \\
\hline
\end{tabular}

The powders were carefully directed to the bottom of the flasks to ensure that all the powder participates in the reaction and to maximize the reaction yield. Both three-neck round bottom flasks were attached to their respective condensers and attached using adapters to the Schlenk line. All joints were lubricated with octadecene to create an airtight seal for when the vacuum and nitrogen lines became active. Figure 6.2 below is an example of the setup for the cadmium source, while Figure 6.3 is for the selenium source.

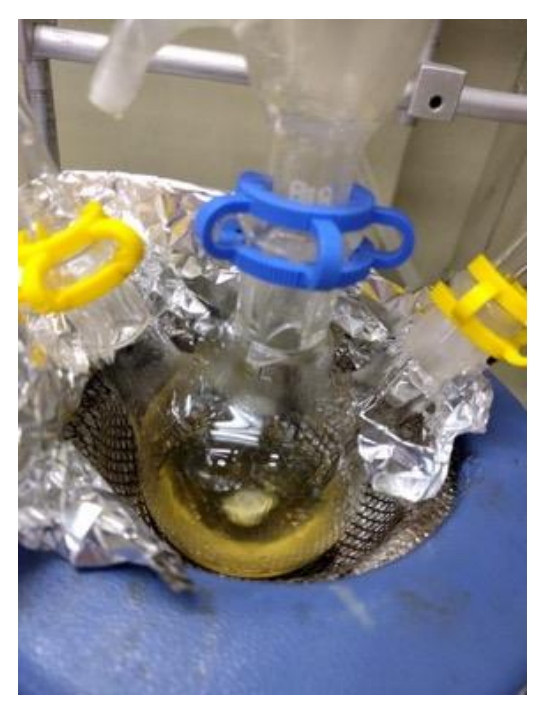

Figure 6.2. Image of the cadmium source for core synthesis.

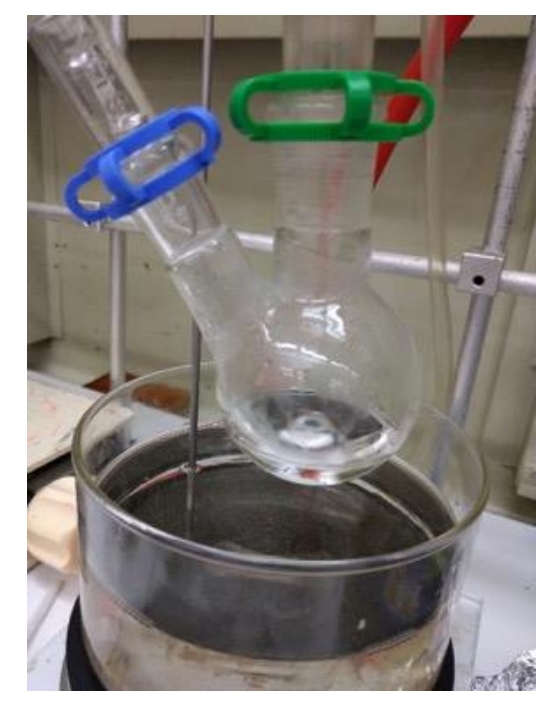

Figure 6.3. Image of the selenium source for core synthesis.

For practical reasons, the synthesis is scaled up. An example of the synthesis work bench setup is shown in Figure 6.4. In this case, the three round bottom flasks in the heating mantles are all triplicate setups of 
cadmium sources, while the leftmost flask in the oil bath had triple the amount required, divided into three portions for the injection stage. Example measurements for the scaled-up synthesis is provided in Tables 6.3 and 6.4 .

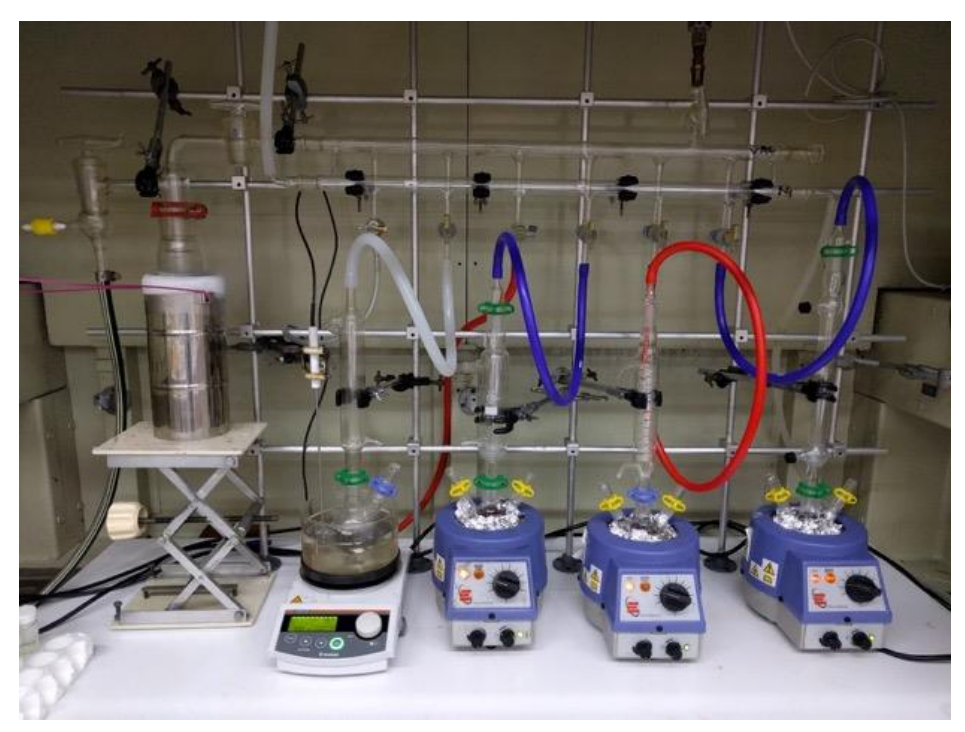

Figure 6.4. Scale up reaction where the three blue heating mantles are triplicate cadmium sources, and the selenium source is triple the amount in the oil bath.

Table 6.3. Example synthesis measurements for preparing the cadmium source, scaled up $3 x$.

\begin{tabular}{|c|c|c|c|}
\hline Compound Name & Cd 1 & Cd 2 & Cd 3 \\
\hline $0.0770 \mathrm{~g} \mathrm{CdO}$ & $0.0783 \mathrm{~g}$ & $0.0773 \mathrm{~g}$ & $0.0788 \mathrm{~g}$ \\
\hline $3.0430 \mathrm{~g}$ Oleic Acid & $3.0538 \mathrm{~g}$ & $3.0601 \mathrm{~g}$ & $3.0433 \mathrm{~g}$ \\
\hline$\sim 15 \mathrm{~mL}$ Octadecene & \multicolumn{3}{|c|}{ Graduated cylinder and pipet } \\
\hline
\end{tabular}

Table 6.4. Example synthesis measurements for preparing the selenium source, scaled up $3 x$.

\begin{tabular}{|c|c|c|}
\hline Compound Name & Amount Needed & Amount Added \\
\hline Se powder & $0.1421 \mathrm{~g}$ & $0.1424 \mathrm{~g}$ \\
\hline Octadecene & $\sim 15 \mathrm{~mL}$ & Graduated cylinder and pipet \\
\hline
\end{tabular}


The injection of the selenium source into the cadmium source to prepare the cadmium selenide quantum dot cores was performed at $220^{\circ} \mathrm{C}$ with the reaction allowed to proceed for $45-90$ seconds before quenching in a cold-water bath. An example of the quenching is shown in Figure 6.5 below.

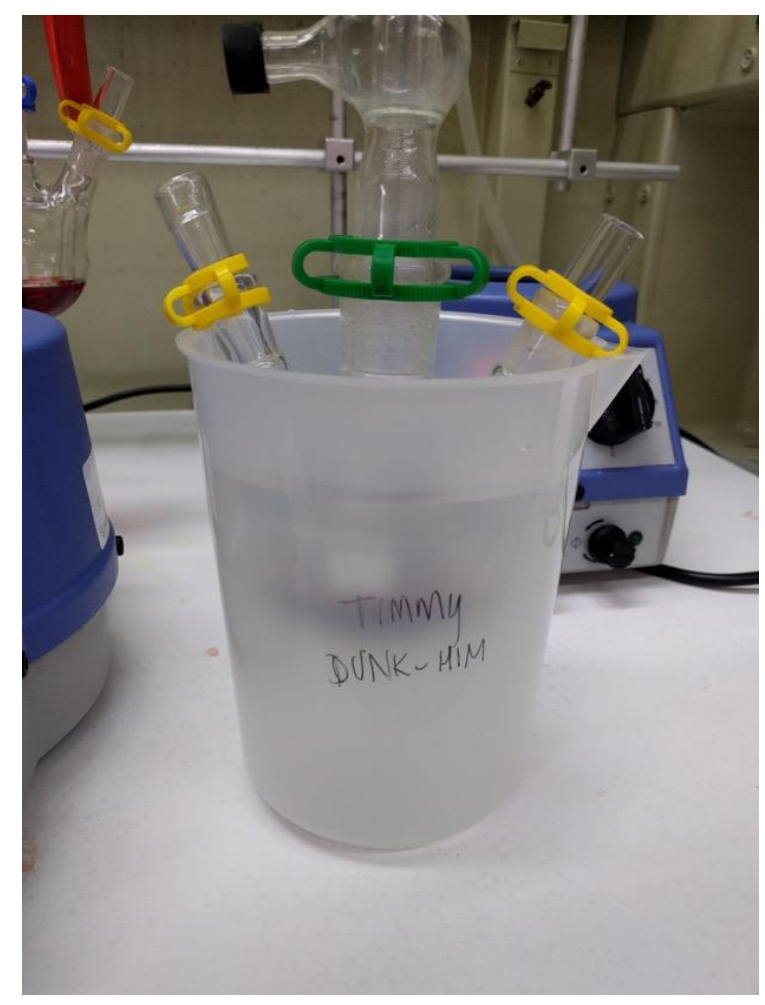

Figure 6.5. The reaction flask is quenched in a cold-water bath to stop the cores from growing too large.

Following along with the triplicate cadmium source scale up preparation from Figure 6.4, the three round bottom flasks were reacted for different time durations, resulting in a deeper, darker color with longer reaction times. This can be observed in Figures 6.6-6.8, where the reaction time allotted was 45 seconds, 60 seconds, and 75 seconds, respectively. 


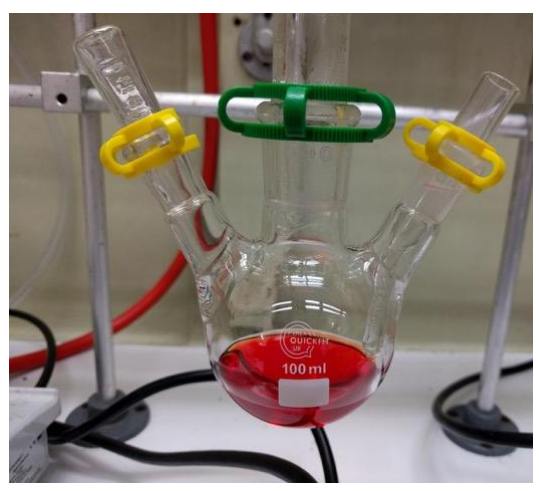

Figure 6.6. A light red color

observed after 45 seconds reaction

time.

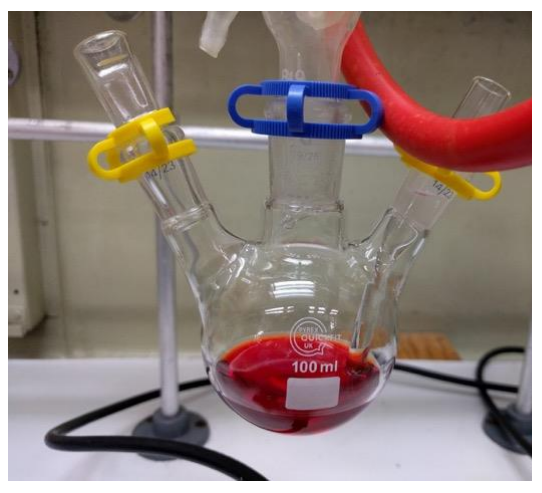

Figure 6.7. A darker red color

observed after 60 seconds reaction

time.

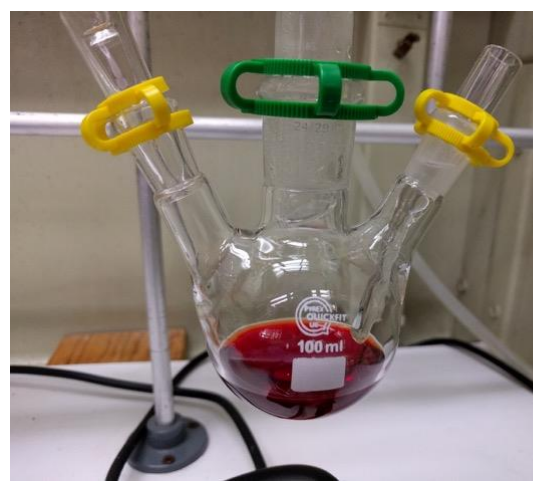

Figure 6.8. A dark burgundy color

observed after 75 seconds reaction

time.

As expected, the longer reaction times resulted in a darker red compared to the brighter reds with less reaction times. Increasing the reaction time allows more time for the quantum dot cores to grow, which is beneficial for improving fluorescence however results in the likelihood of growing the core sizes past the nanoscale. This results in the cores becoming too large, and therefore, they will not characteristically fluorescence since they are no longer exhibiting nanoparticle behavior. Figure 6.9 below compares all three quantum dot cores after injection.

In addition, Figure 6.10 is a case where the quantum dot cores grew for a duration that encourages particle growth past the nanoscale. The result is a blackened structure, where upon irradiation by a fluorescent flashlight, no fluorescence is observed. These cores are colloquially termed as "dead" cores and are of no use since they no longer are at the nanoscale. 


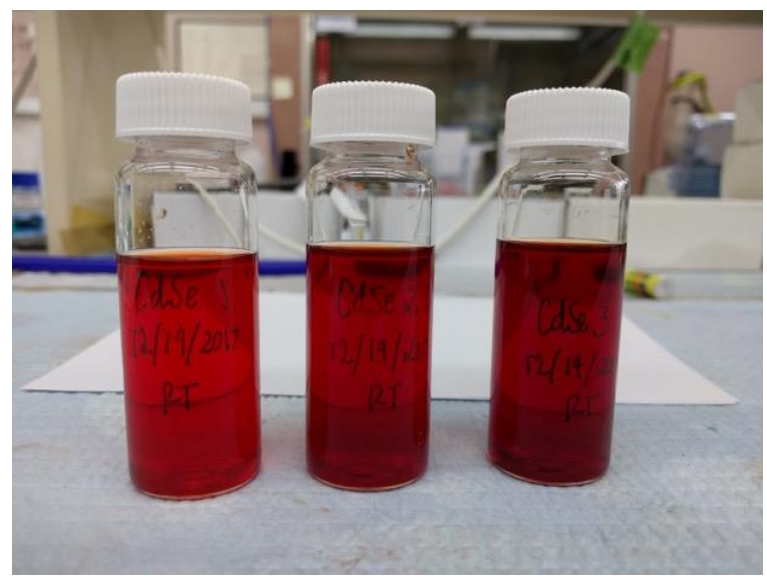

Figure 6.9. A side by side comparison of color

differences based on reaction time duration.

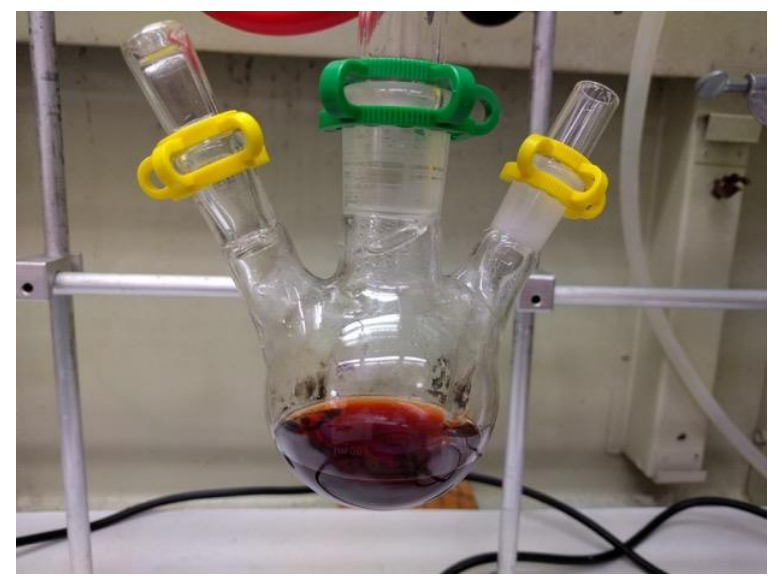

Figure 6.10. The color is far too dark, an almost blood red color, resulting in excessive growth, and cores do not fluoresce.

The raw mixture must be purified and centrifuged in the next step since the raw mixture is not purified and contains remainder contaminants from the core synthesis. Figures 6.11 and 6.12 below shows an example where the raw quantum dot mixture is immiscible with the chloroform solvent in a typical cuvette. This marks a major milestone with the next one purifying and centrifuging the cores such that the quantum dot $\begin{array}{llll}\text { mixture } & \text { miscible with thentoroform }\end{array}$ 


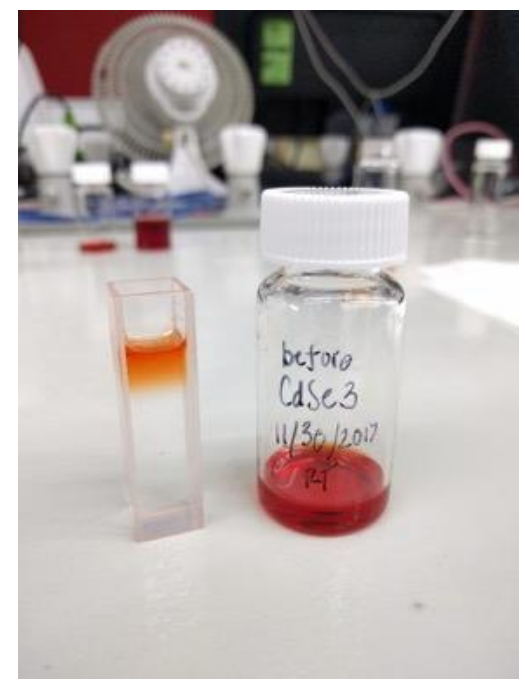

Figure 6.11. The raw quantum dot mixture is added into chloroform, but the system is immiscible in the solvent.

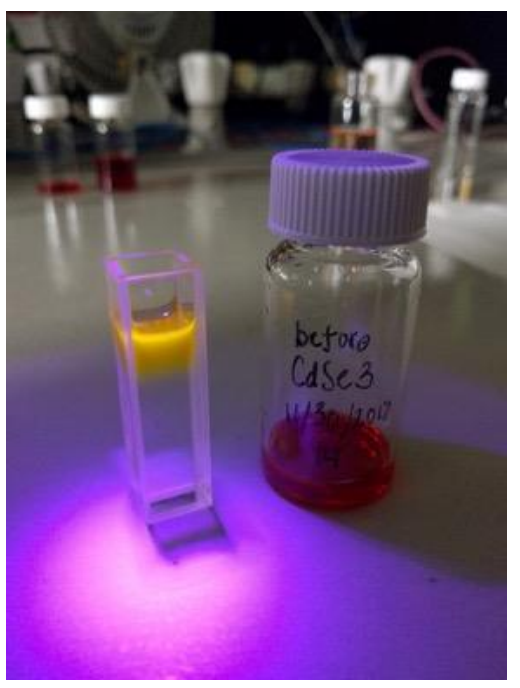

Figure 6.12. Quantum dots fluoresce under a 395 nm UV source, verifying they were synthesized correctly.

\section{$\underline{\text { 6.1 Centrifugation and Purification of CdSe Cores }}$}

A roadmap of the centrifugation step is provided in Figure 6.13 with the major steps outlined throughout the process. 


\section{Split raw mixture into centrifuge tubes}

\section{Add $5 \mathrm{~mL}$ chloroform, $6 \mathrm{~mL}$ acetone, 12.5 $\mathrm{mL}$ ethanol}

\section{Weigh and balance centrifuge tubes}

\section{Centrifuge at 17000 rpm for 13 minutes}

\section{Discard supernatant and repeat}

Figure 6.13. Centrifugation roadmap for purifying the cadmium selenide quantum dot cores.

After the cores are synthesized, the mixture is promptly washed and purified by solvent miscibility using a solvent mixture of $5 \mathrm{~mL}$ chloroform, $6 \mathrm{~mL}$ acetone, and $12.5 \mathrm{~mL}$ ethanol. The raw CdSe quantum dot cores mixture is transferred into Falcon centrifuge tubes before adding the respective volumes of solvents above. The tubes were then centrifuged in a Beckman Coulter Allegra 64 benchtop centrifuge at 17000 rpm for 13 min. After the first run, the supernatant was discarded before the same purification mixture was added to the precipitate. The tube was then shaken thoroughly and recentrifuged a second time; this process was repeated a total of three times.

After discarding the third supernatant, the final precipitate in one tube was dissolved in $10 \mathrm{~mL}$ chloroform. The precipitate went into mixture, and the resulting mixture was transferred from one tube to another until all the precipitate was redissolved in chloroform. The quantum dots in mixture were then transferred into a clean vial for shelling. 
The purification and centrifugation steps are based upon using different polarity solvents to wash and rinse the raw quantum dot cores mixture to separate the purified cores and isolate them from the oils and unnecessary solvents such as octadecene and have them finalized in chloroform solvent. This begins with obtaining the necessary solvents used in the "purification mixture," including technical grade chloroform, acetone, and ethanol. In addition, a graduated cylinder is helpful along with a calibrated pipet to deposit the chloroform at the final step.

The first step is to take the raw quantum dot mixture and separate into the Falcon centrifuge tubes as equally as possible. This is accomplished using a plastic pipette and estimating by eye. An example of the separation is shown in Figure 6.14.

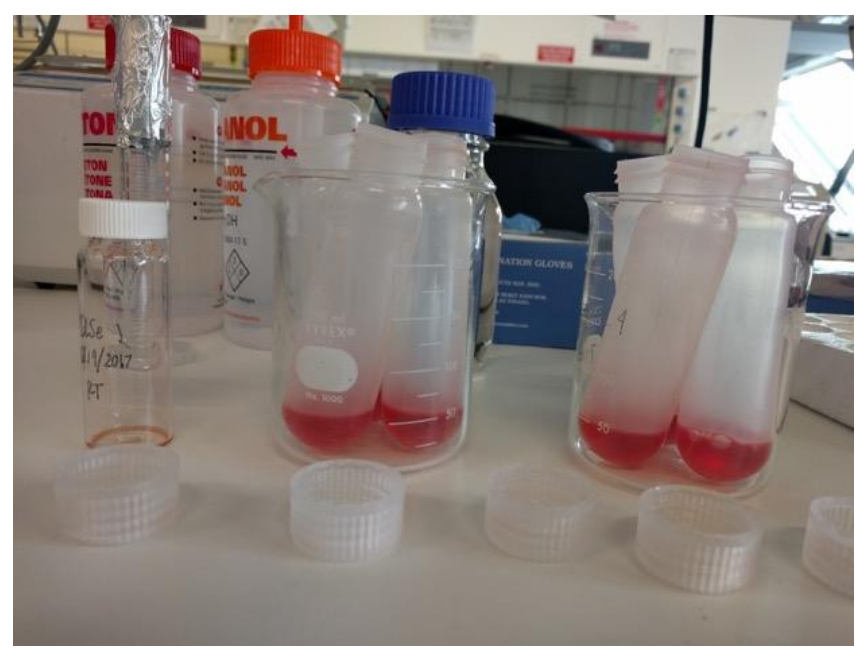

Figure 6.14. The raw quantum dot mixture is transferred equally into 6 Falcon centrifuge tubes.

After separation, the combination of solvents was added with rigorous shaking and mixing between additions. First, $5 \mathrm{~mL}$ of chloroform was added to each tube, followed by $6 \mathrm{~mL}$ of acetone, and finally 12.5 $\mathrm{mL}$ ethanol. The addition of each solvent into the tubes is shown in Figures 6.15-6.17, respectively. 


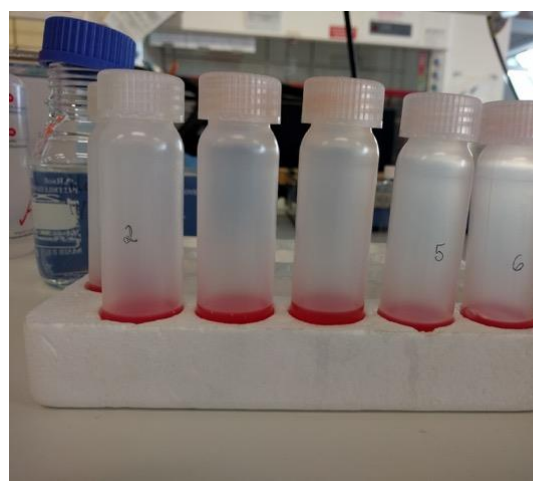

Figure 6.15. The mixture after

addition of $5 \mathrm{~mL}$ chloroform.

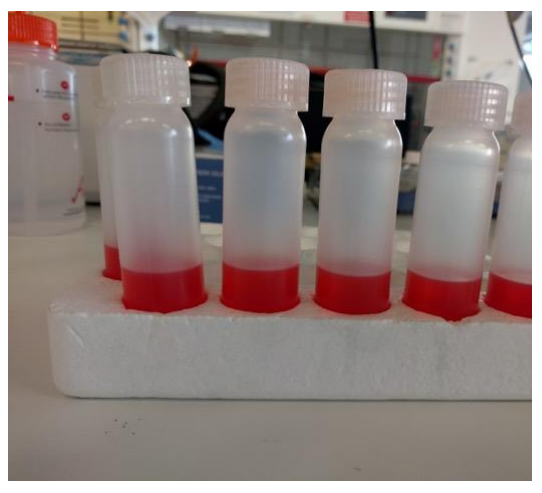

Figure 6.16. The mixture after

addition of $6 \mathrm{~mL}$ acetone.

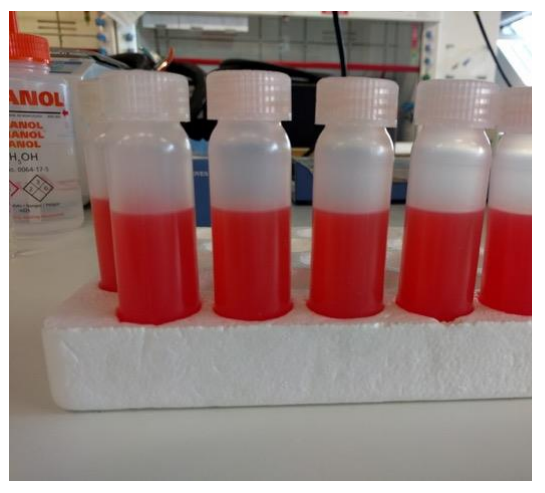

Figure 6.17. The mixture after

addition of $12.5 \mathrm{~mL}$ ethanol.

After the purification mixtures are added, each tube was weighed on a scale to determine the mass of the mixture inside the tube. Before entering the centrifuge, each tube should be roughly the same weight - this is accomplished by topping off each tube with additional ethanol if necessary. Once all the tubes have been equalized, the tubes were placed inside a Beckman Coulter Allegra 64 benchtop centrifuge as shown in Figures 6.18-619 with the cap screwed firmly on before starting the centrifuge at $17000 \mathrm{rpm}$ for 13 minutes. 


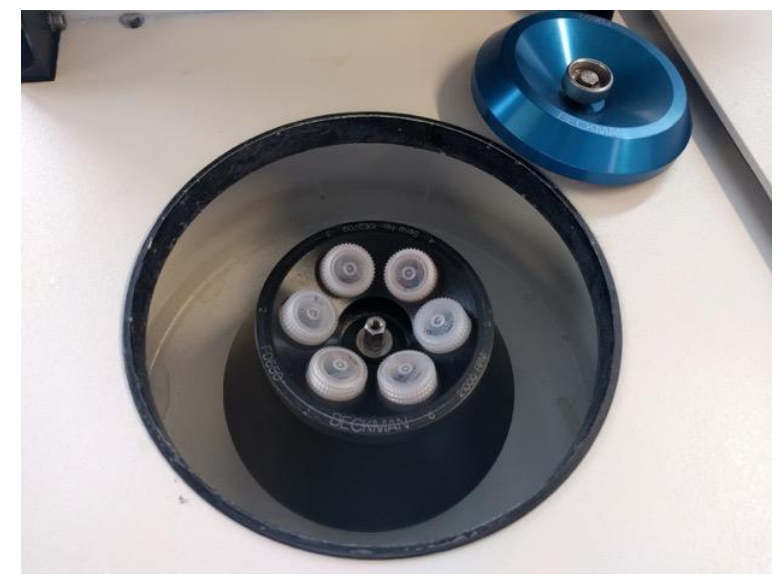

Figure 6.18. All 6 Falcon centrifuge tubes loaded into the benchtop centrifuge.

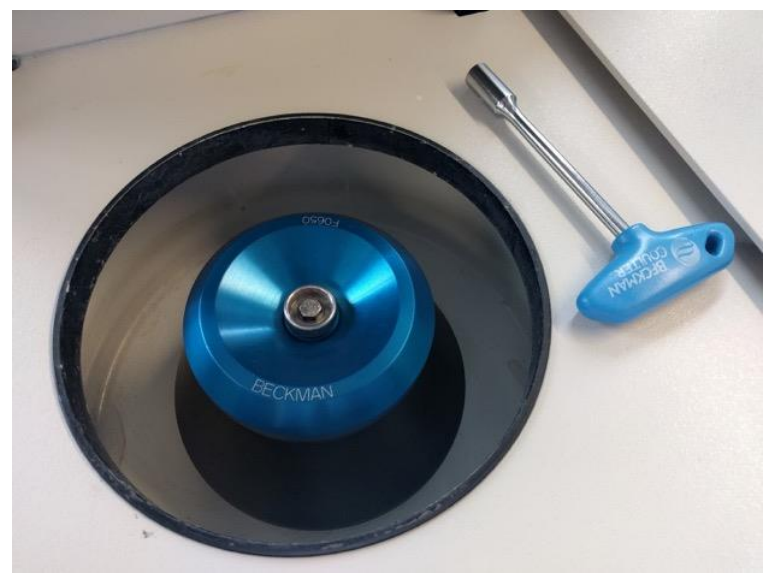

Figure 6.19. The cap was screwed on afterwards

tightly using the hand tool before running the

After centrifugation, the resulting precipitates appeared on the sides and at the bottom of the centrifuge tubes as shown in Figure 6.20.

Discarding the supernatant shows the effect of the purification mixture in removing the unneeded oils and dirty reagents from the quantum dot raw mixture. This is shown in Figure 6.21 below where the distinct yellow color is characteristic of the cadmium precursor from before. In addition, there is a slight slimy texture to it that is reminiscent of the octadecene carrier solvent. The strong color and consistency of the supernatant verifies the success of the purification mixture in removing the impurities from the quantum dot mixture. 


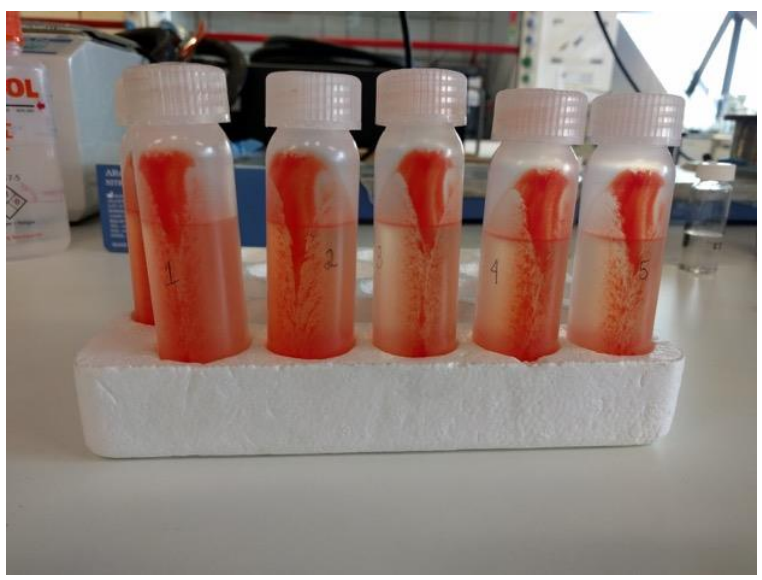

Figure 6.20. The nanoparticles separated and were stuck to the walls of the centrifuge tubes.

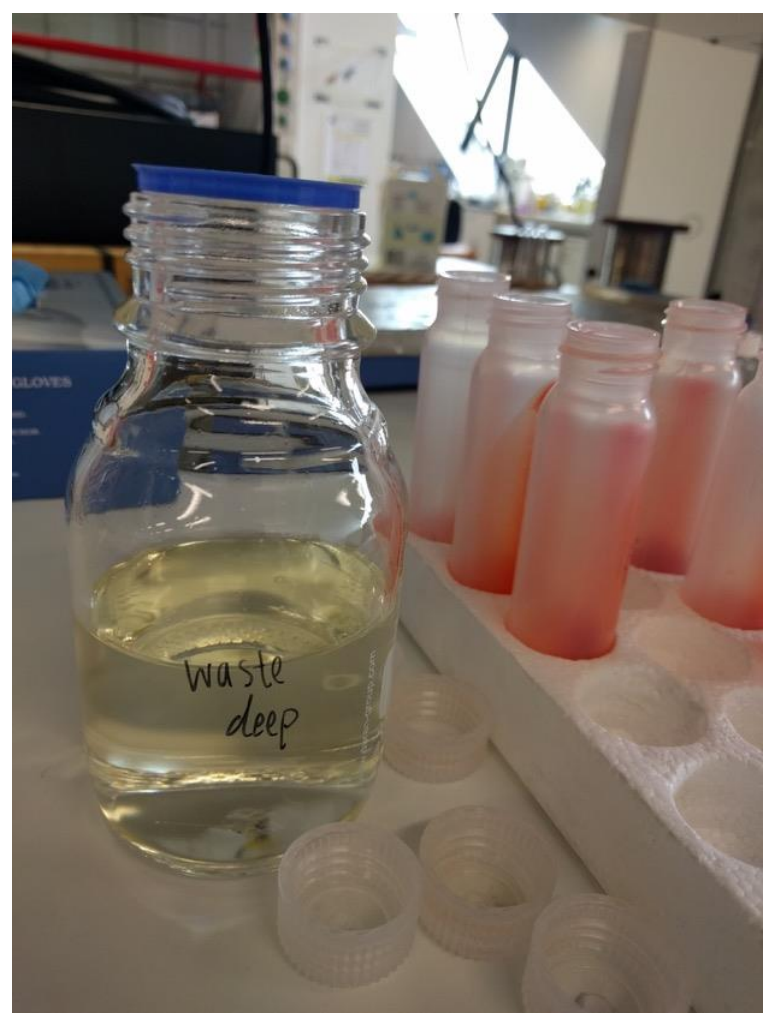

Figure 6.21. The first supernatant was very

yellow, indicating collection and purification of raw mixture from impurities.

The centrifugation step was then repeated with the addition of $5 \mathrm{~mL}$ chloroform to redissolve the quantum dot cores precipitate before adding the acetone and ethanol portions. Again, the mixtures were thoroughly mixed and weighed to ensure balance before another cycle in the centrifuge at $17000 \mathrm{rpm}$ and 13 minutes. The precipitate pattern was different the second time around as shown in Figure 6.22, suggesting that the separation of the precipitate from the solvent was easier and that there are less contaminants present in the tube. In addition, the supernatant is now much clearer and does not have the yellow tint previously. A side by side comparison of the color disparity is shown in Figure 6.23, where the left bottle contains the first supernatant and the right the second supernatant. 


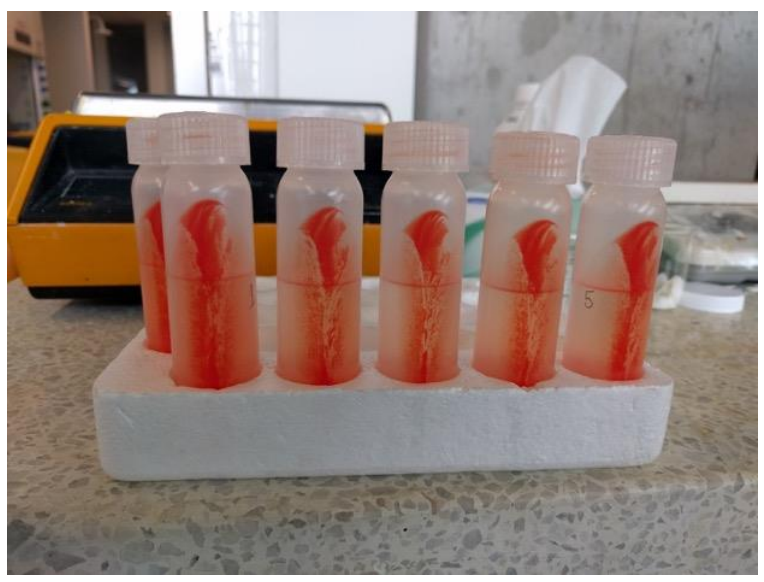

Figure 6.22. The second centrifuge had a

different, cleaner precipitate fan pattern

compared to before.

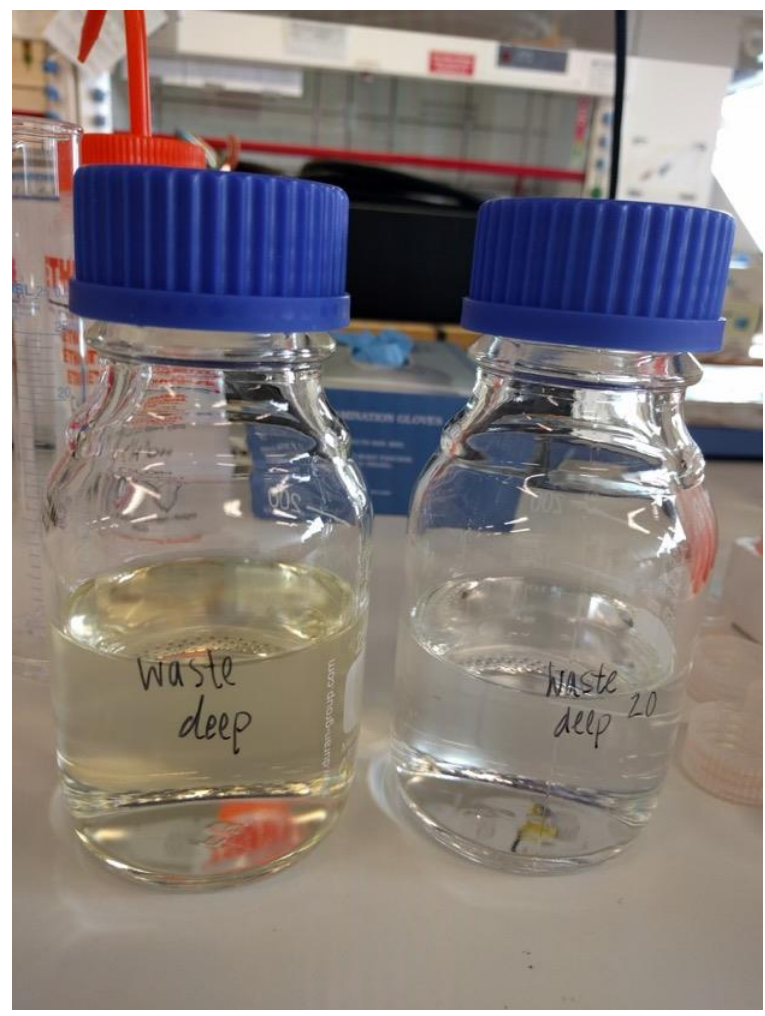

Figure 6.23. A comparison of supernatants - the second is considerably clearer and cleaner than the first.

The centrifuge process was repeated one final time, resulting in again a different precipitate fan pattern as shown in Figure 6.24. Compared to the previous two patterns, this pattern has a much smoother transition and cleaner gradient from top to bottom. 


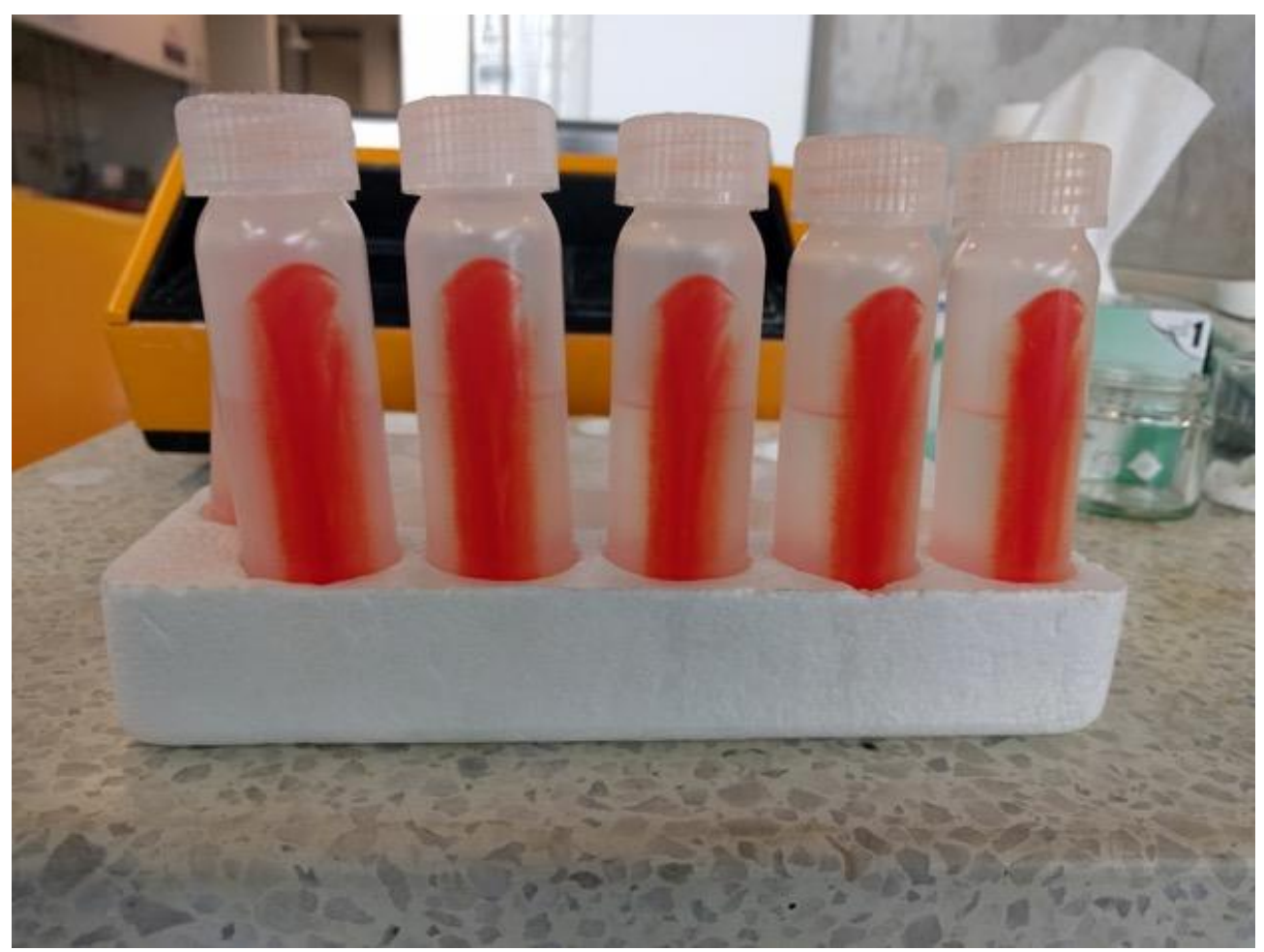

Figure 6.24. The final precipitate fan pattern had a very clean and consistent gradient throughout all centrifuge tubes.

At this point, the cadmium selenide quantum dot cores have been purified and exist as a solid cluster at the bottom of the collection of centrifuge tubes. Using a calibrated pipet, $5 \mathrm{~mL}$ of chloroform was deposited into one of the tubes to redissolve the precipitate. This new mixture of cadmium selenide quantum dot cores suspended in chloroform was then transferred into another tube to then dissolve that precipitate. This procedure was repeated until all six tubes and their respective precipitate was collected into one centrifuge tube. The final mixture was then transferred to a scintillation vial and labeled until the next step.

\subsection{Optical Characterization Results for the CdSe Cores}

The following figures are UV-Vis measurements performed on the Cary UV-Vis-NIR 5G spectrophotometer for $100 \mu \mathrm{L}$ of the quantum dot cores mixture into $3 \mathrm{~mL}$ chloroform. Figure 6.25 overlays the UV-Vis measurements amongst the three systems to show differences in absorbance intensity and the peak shift that is observed as reaction time is increased. 


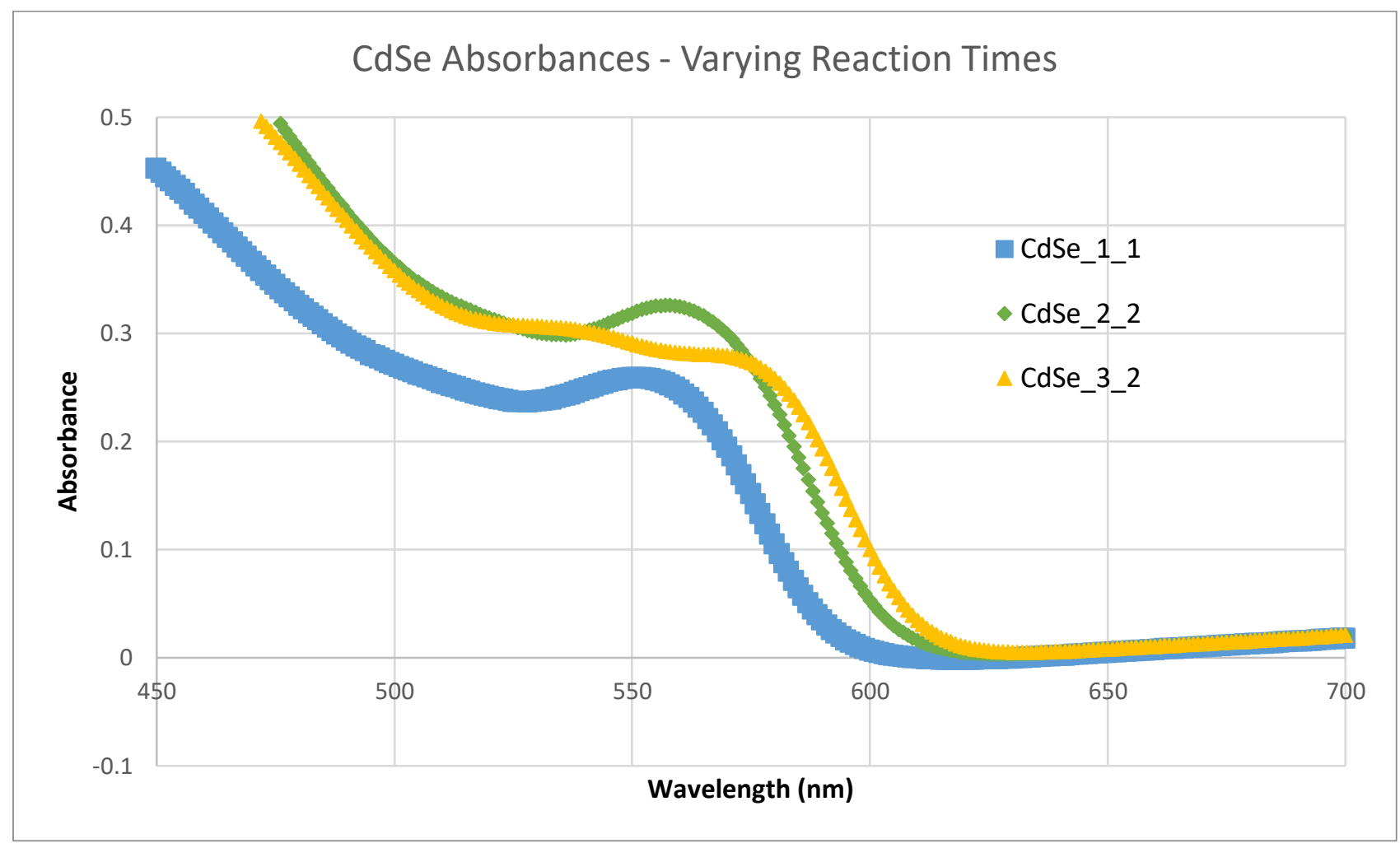

Figure 6.25. UV-Vis measurement overlay for the varying reaction times for CdSe cores.

From this data, it is observed that increasing the reaction time slightly redshifts the wavelength that is absorbed. Longer reaction times result in larger cores that express longer wavelengths. These wavelengths have less energy because of a smaller band gap. One interesting observation is that CdSe System 3, with a reaction time of 75 seconds, has two prominent absorbance peaks. This is perhaps due to the injection process not being as efficient as possible. Perhaps the injection occurred too slowly resulting in a distribution of particle sizes rather than a clean single peak and therefore a more consistent particle size distribution. Despite this conclusion, the two previous reaction times were very successful.

\subsection{Calculating the Amounts of $\mathrm{Zn}$ and $\mathrm{S}$ Injection Precursors}

Optical characterization measurements were acquired for the purified cores to estimate the amounts of zinc and sulfur components necessary for the shelling procedure. This was accomplished using estimations from 
calculations from $\mathrm{Wu}$ and Jasieniak ${ }^{[37,38]}$. The results of Equations 1 and 2 were averaged to determine the average CdSe core diameter. For these equations, the wavelength of the UV-Vis measurement is important in solving these equations. The molar extinction coefficient can be determined by substituting in the known value for the E1s transition in $\mathrm{CdSe}$ as $2.30 \mathrm{eV}$ for Equation $3^{[22]}$. Additionally, for Equation 4, the absorbance value from the UV-Vis measurement is required to determine the bulk concentration of the quantum dot cores ${ }^{[22]}$. These values are substituted into the equations to determine the molar amount of either zinc or sulfur required to successfully and fully coat the cores structure.

$$
\begin{array}{cc|}
D(\mathrm{~nm})=59.60816-0.54736 \lambda+1.8873 \times 10^{-3} \lambda^{2}- & \text { Equation 1 } \\
2.85743 \times 10^{-6} \lambda^{3}+1.62974 \times 10^{-9} \lambda^{4} & \\
D=\left(1.6122 \times 10^{-9}\right) \lambda^{4}-\left(2.6575 \times 10^{-6}\right) \lambda^{3}+ & \text { Equation 2 } \\
\left(1.6242 \times 10^{-3}\right) \lambda^{2}-(0.4277) \lambda+(41.57) & \\
\varepsilon_{1 S}\left(\mathrm{M}^{-1} \mathrm{~cm}^{-1}\right)=155507+6.67054 \times 10^{13} \exp \left(-\frac{E_{1 S}}{0.10551}\right) & \text { Equation 3 } \\
& \\
{[\mathrm{CdSe}](\mathrm{M})=\frac{\mathrm{Abs}}{l(\mathrm{~cm}) \varepsilon_{1 S}\left(\mathrm{M}^{-1} \mathrm{~cm}^{-1}\right)} \times \frac{\Delta E_{1 S, \mathrm{HWHM}}(\mathrm{eV})}{0.06}} & \text { Equation 4 }
\end{array}
$$

Since the $\mathrm{ZnS}$ monolayer shell has an estimated thickness of $0.31 \mathrm{~nm}$, every additional shell can simply be added on top of the average calculated CdSe core diameter. By calculating the difference between the inner (core only) and outer (shell) volumes, the change in volume between the layers can be converted to the number of moles of $\mathrm{ZnS}$ present in the shell sufficient to fully shell the core with a complete layer. Finally, multiplying the number of moles by the molar mass of $\mathrm{ZnO}$ will determine the mass of $\mathrm{ZnO}$ needed. Furthermore, this mass can be transformed into density as the injection volume when injecting the zinc and 
sulfur precursors over the cores. The volumes for the zinc and sulfur precursors will be the same since they have the same stoichiometric ratio and are equimolar.

Table 6.5 provides an example shelling calculation for a CdSe core system where the determined absorption was at $\mathbf{5 5 8} \mathbf{~ n m}$ with an absorbance at $\mathbf{0 . 3 8 3}$. Solving the above equations results in an estimated core diameter of $3.289 \mathrm{~nm}$.

Table 6.5. Determining the Change in Volume Between Inner and Outer Spheres

\begin{tabular}{|c|c|c|c|}
\hline Shell & Inner Diameter $(\mathbf{n m})$ & Shell Diameter (nm) & $\Delta$ Volume (cm $\left.\mathbf{c}^{\mathbf{3}}\right)$ \\
\hline 1 & $3.289 \mathrm{~nm}$ & $3.909 \mathrm{~nm}$ & $1.26 \times 10^{-20} \mathrm{~cm}^{3}$ \\
\hline 2 & $3.909 \mathrm{~nm}$ & $4.529 \mathrm{~nm}$ & $1.74 \times 10^{-20} \mathrm{~cm}^{3}$ \\
\hline 3 & $4.529 \mathrm{~nm}$ & $5.149 \mathrm{~nm}$ & $2.28 \times 10^{-20} \mathrm{~cm}^{3}$ \\
\hline
\end{tabular}

The change in volume $(\Delta \mathrm{V})$ is then multiplied by the calculation for the bulk concentration, which gives the number of moles required to completely and ideally shell the quantum dot cores. The molar amount is used to determine the grams of either zinc oxide or sulfur precursor to be prepared. The composition of the zinc precursor includes zinc oxide, and 2 molar equivalents the total molar amount for zinc oxide for the addition of oleic acid. These volumes are added together, and the difference of the summed volume from 5 $\mathrm{mL}$ is filled using octadecene. The sulfur precursor simply adds the sulfur powder with $5 \mathrm{~mL}$ octadecene. Table 6.6 below summarizes the molar amounts of each component required in preparing the zinc and sulfur shelling precursors.

Table 6.6. Converting $\Delta V$ to Mass to Determine Shelling Injection Volumes

\begin{tabular}{|c|c|c|c|c|c|}
\hline Shell & $\begin{array}{c}\text { Mol ZnS } \\
\text { Required }\end{array}$ & Mass ZnO (g) & $\begin{array}{c}\text { ZnO Injection } \\
\text { Volume (mL) }\end{array}$ & Mass S (g) & $\begin{array}{c}\text { S Injection } \\
\text { Volume (mL) }\end{array}$ \\
\hline 1 & $3.33 \times 10^{-4} \mathrm{~mol}$ & $0.0271 \mathrm{~g}$ & $1.20 \mathrm{~mL}$ & $0.0107 \mathrm{~g}$ & $1.20 \mathrm{~mL}$ \\
\hline 2 & $4.58 \times 10^{-4} \mathrm{~mol}$ & $0.0373 \mathrm{~g}$ & $1.64 \mathrm{~mL}$ & $0.0147 \mathrm{~g}$ & $1.64 \mathrm{~mL}$ \\
\hline
\end{tabular}




\begin{tabular}{|c|c|c|c|c|c|}
\hline 3 & $6.02 \times 10^{-4} \mathrm{~mol}$ & $0.0490 \mathrm{~g}$ & $2.16 \mathrm{~mL}$ & $0.0193 \mathrm{~g}$ & $2.16 \mathrm{~mL}$ \\
\hline \multirow{2}{*}{ TOTAL } & $1.393 \times 10^{-3}$ & $0.1134 \mathrm{~g}$ & $5 \mathrm{~mL}$ & $0.0447 \mathrm{~g}$ & $5 \mathrm{~mL}$ \\
& $\mathrm{~mol}$ & & & \\
\hline
\end{tabular}

In addition, 2 molar equivalents $\sum \mathrm{ZnS}=1.393 \times 10^{-3} \mathrm{~mol} \times 282.46 \mathrm{~g} / \mathrm{mol}=0.7869 \mathrm{~g}$ or $0.88 \mathrm{~mL}$

\subsection{Shelling the CdSe Cores with ZnS Shells}

The second stage in preparing the core-shell quantum dots is to shell the cores that were previously synthesized. This is similarly accomplished in preparing a zinc source and a sulfur source, alternating injections to form the zinc sulfide shells around the cadmium selenide quantum dot cores. However, it is important to mention that a precise amount of zinc and sulfur sources must be injected so that the cores are sufficiently shelled - adding too much will result in excess off the surface of the core while too little will result in an incomplete coating around the cores. For this purpose, the shelling technique is very sensitive in constructing high quality and high performing core-shell quantum dot structures.

Now with the quantum dot cores mixture purified and suspended in pure chloroform, the optical characterization that was previously unobtainable can be performed. The results of the varied reaction times are shown in ambient lighting and under UV source irradiation in Figures 6.26-6.31 below. 


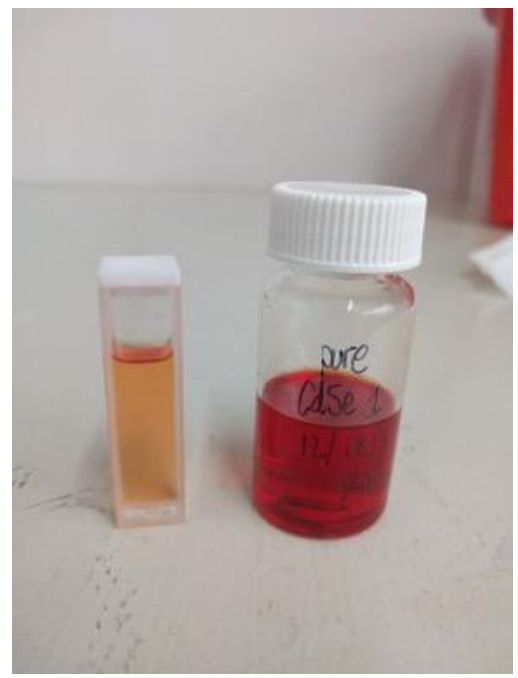

Figure 6.26. The 45 seconds reaction time mixture is now miscible with chloroform.

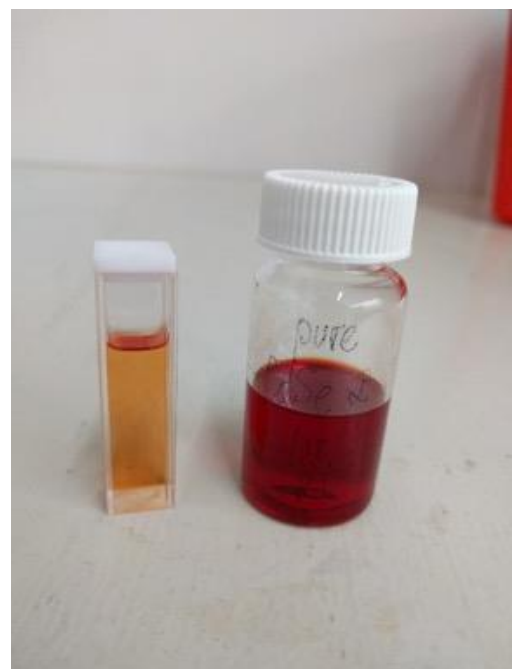

Figure 6.27. The 60 seconds reaction time mixture is now miscible with chloroform.

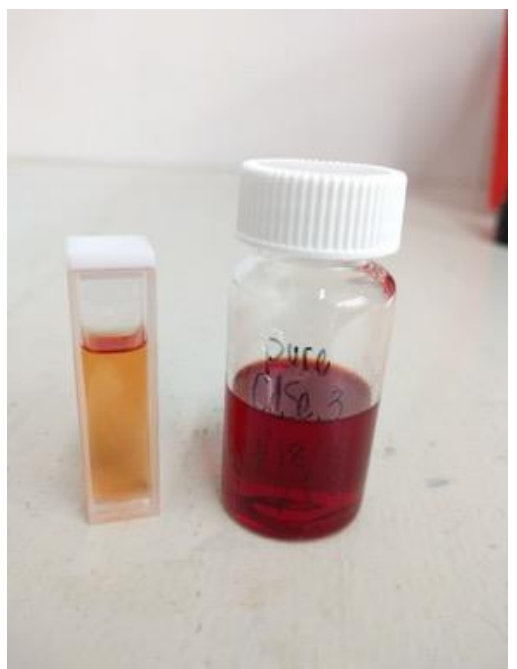

Figure 6.28. The 75 seconds reaction time mixture is now miscible with chloroform. 


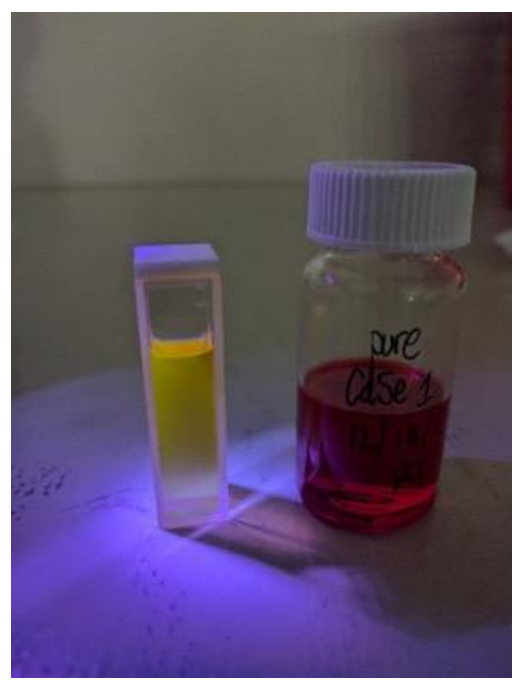

Figure 6.29. The 45 seconds

batch fluoresces a pale-yellow color.

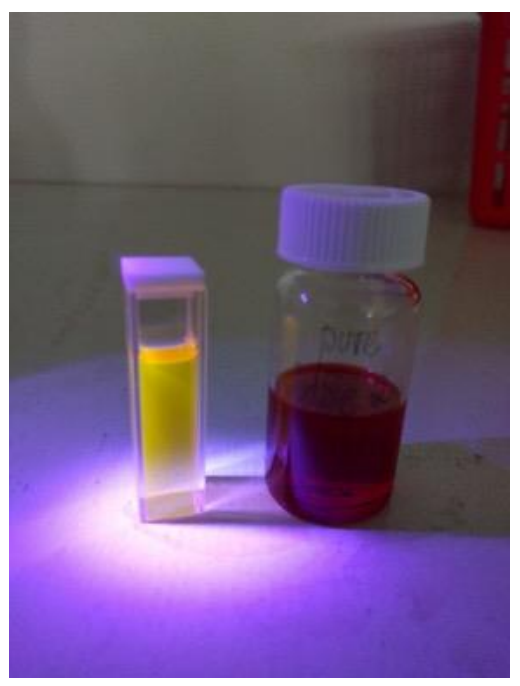

Figure 6.30. The 60 seconds

batch fluoresces a deeper yellow color.

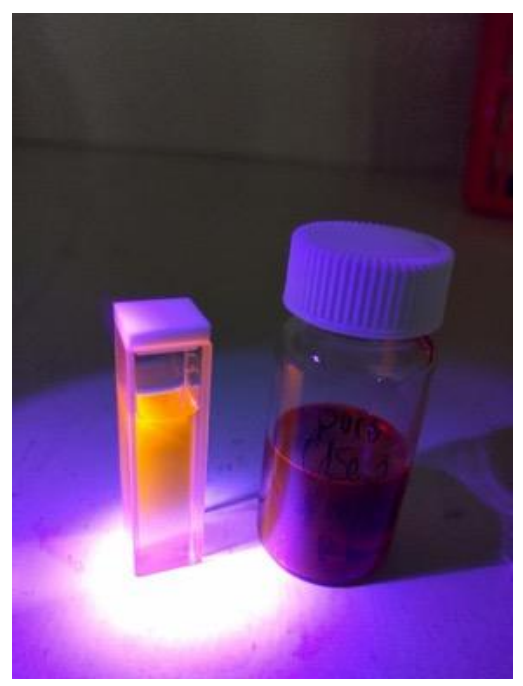

Figure 6.31. The 75 seconds

batch fluoresces a bright orange

color.

From the images above, it is observed that a greater reaction time results in the fluoresced color becoming more orange and less pale yellow. Ad the particles become larger and larger, they fluoresce a larger wavelength with lower energy due to a smaller band gap energy. 


\section{Core-Shell Synthesis - Making the CdSe/ZnS Core-Shell Quantum Dots}

A roadmap of the shelling step is provided in Figure 7.1 with the major steps outlined throughout the process.

\section{Determine amounts of $\mathrm{Zn}$ and S precursors} from Equations 1-4

\section{Add octadecene and flush out chloroform from cores}

\section{Prepare Zn and S shelling precursors}

\section{Alternate injections for $\mathrm{Zn}$ and S}

\section{Centrifuge finished core-shell CdSe/ZnS} quantum dots

Figure 7.1. Synthesis roadmap for shelling the CdSe cores with Zns shells.

The zinc precursor is prepared by adding the estimated amount of zinc oxide, oleic acid, and octadecene into a three-neck round bottom flask with stirring bar. The resulting mixture s allowed to mix thoroughly under ambient conditions for ten minutes before proceeding to degas the mixture at $110^{\circ} \mathrm{C}$ for 1 hour. The vacuum is then switched to nitrogen flow, and the heating mantle is adjusted to $260^{\circ} \mathrm{C}$ for an additional hour. Afterwards, the mixture is cooled down to $110^{\circ} \mathrm{C}$ and evacuated under vacuum for an additional 30 minutes. Finally, the zinc precursor mixture is heated and left at $180^{\circ} \mathrm{C}$ under nitrogen flow. The final mixture should be a clear milky off-white color. 
The sulfur precursor is prepared by adding the estimated amount of sulfur powder and octadecene to another three-neck round bottom flask with stirring bar. The resulting mixture is allowed to mix thoroughly under ambient conditions for ten minutes before proceeding to degas the mixture at $110^{\circ} \mathrm{C}$ for 1 hour. The vacuum is then switched to nitrogen flow, and the heating mantle is adjusted to $180^{\circ} \mathrm{C}$ for an additional hour. The mixture should be a clear yellow mixture and left at $180^{\circ} \mathrm{C}$ under nitrogen flow until proceeding to shelling.

Before injecting to shell the CdSe cores, the purified CdSe mixture was transferred to a three-neck round bottom flask with stirring bar and slowly heated to $100^{\circ} \mathrm{C}$ under nitrogen flow. $30 \mathrm{~mL}$ of octadecene was then added, and one neck of the round bottom flask was left open so that the chloroform can escape. After an hour of allowing the chloroform to escape, the mixture was evacuated under vacuum for 1 hour at $100^{\circ} \mathrm{C}$ before switching back to nitrogen flow. The cores were heated at $220^{\circ} \mathrm{C}$ prior to shelling.

Shelling the CdSe cores with $\mathrm{ZnS}$ shells occurs by alternating the zinc or sulfide injections according the predicted volumes from solving Equations 1-4. Injections are different than core synthesis in that they are to be done much slower - drip wise over a minute is ideal. After the injection of the first zinc shell, allow for 15 minutes before injecting the first sulfur shell - the result is the first completed zinc sulfide ZnS shell. Allow 15 minutes before each shell injection and leave the final product overnight stirring without heat to complete the shelling procedure. The end result is a $\mathrm{CdSe} / \mathrm{ZnS}$ core-shell quantum dot mixture.

With these numbers calculated, the next step is to prepare the zinc and sulfur precursors for the shelling procedure. An example of the benchtop for shelling is provided in Figure 7.2 - the key is to set up and organize the workspace such that there is a dedicated vacuum and nitrogen line for both the zinc and sulfur precursors along with the cores that are to be shelled.

Table 7.1 below is an example experimental setup using the numbers above in preparing the zinc and sulfur shelling precursors. 
Table 7.1. Example Setup for Shelling the CdSe Cores - Abs 0.383 at $558 \mathrm{~nm}$

\begin{tabular}{|c|c|c|}
\hline Compound Name & Amount Needed & Amount Added \\
\hline $\mathrm{ZnO}$ & $0.1134 \mathrm{~g}$ & $0.1158 \mathrm{~g}$ \\
\hline Oleic acid & $0.7873 \mathrm{~g}$ & $0.8049 \mathrm{~g}$ \\
\hline Octadecene & $\sim 3.20 \mathrm{~mL}$ & $\sim 3.20 \mathrm{~mL}$ by graduated cylinder \\
\hline S powder & $0.0447 \mathrm{~g}$ & $0.0455 \mathrm{~g}$ \\
\hline Octadecene & $\sim 5 \mathrm{~mL}$ & $\sim 5 \mathrm{~mL}$ by graduated cylinder \\
\hline
\end{tabular}

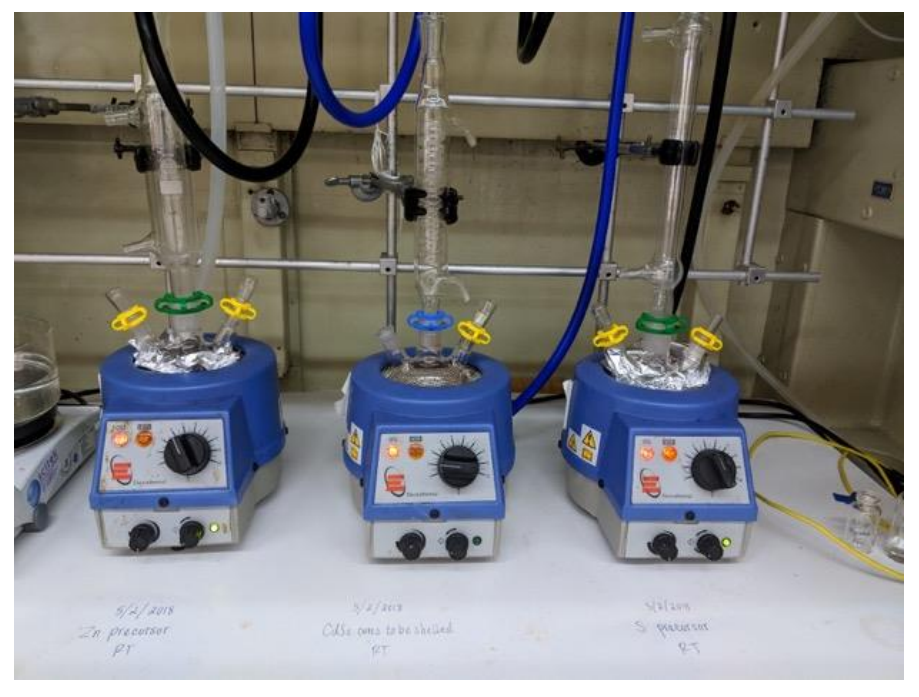

Figure 7.2. Benchtop setup with the cores in the middle, leftmost is the Zn precursor, and rightmost S precursor.

$\sim 20 \mathrm{~mL}$ of octadecene was added to the middle cores mixture that is to be shelled. One of the necks is left open to ambient conditions such that the chloroform carrier solvent can escape and not travel into the vacuum line. Ideally, the goal of the nitrogen flow and slight heat at $80^{\circ} \mathrm{C}$ should be sufficient to transfer the suspended cores from the chloroform into the octadecene. Chloroform should be given at least an hour 
to fully escape to avoid getting pulled up into the vacuum lines. Figure 7.3 is the round bottom flask immediately after the addition of octadecene, while Figure 7.4 is after half an hour where it is observed that the volume decreases with the loss of chloroform.

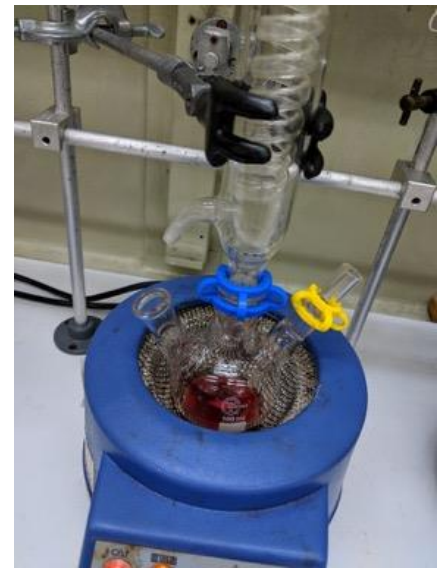

Figure 7.3. The CdSe cores flask has an open neck so that the chloroform can escape.

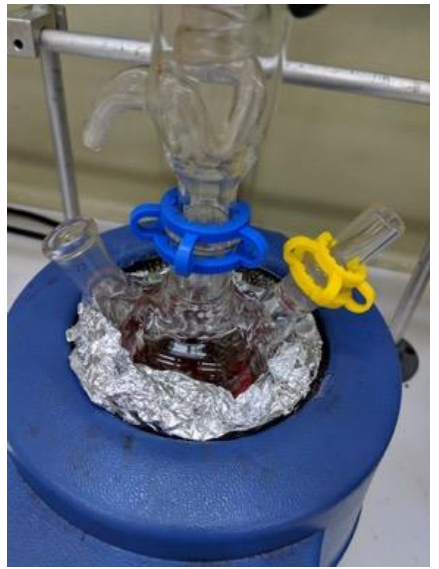

Figure 7.4. After 1 hour of allowing the chloroform to escape, the volume of the mixture is less than before.

With the cores prepared into the octadecene and the chloroform out of the system, the zinc and sulfur shelling precursors were prepared accordingly to the estimated ratios from the calculations from $\mathrm{Wu}$ and Jasieniak. The shelling precursors are shown in Figure 7.5 for the zinc precursor and in Figure 7.6 for the sulfur precursor. 


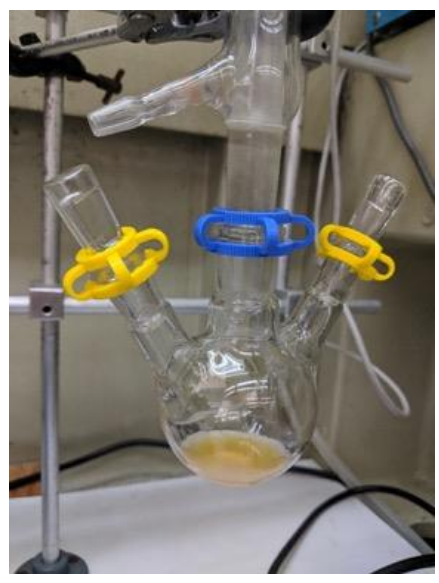

Figure 7.5. The zinc shelling precursor ready for alternating injection to generate the $\mathrm{ZnS}$ shells.

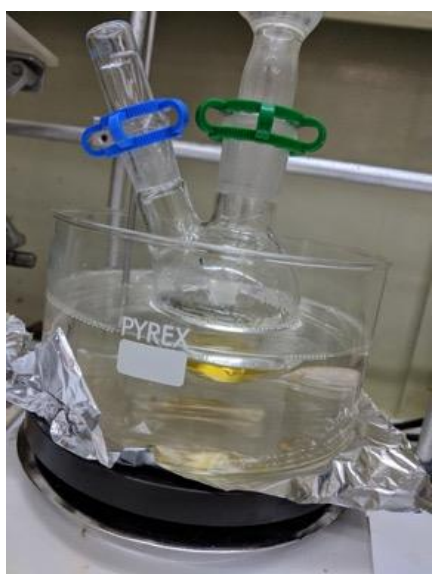

Figure 7.6. The sulfur shelling precursor ready for alternating injection to generate the $\mathrm{ZnS}$ shells.

Injections were performed according to the experimental procedures with the zinc and sulfur shelling precursors alternating with slow dropwise addition to the quantum dot cores mixture heated at $220^{\circ} \mathrm{C}$. Each shell reacted for at least 15 minutes before proceeding to the next shelling component. Figure 7.7 shows the completed raw mixture for the core-shell $\mathrm{CdSe} / \mathrm{ZnS}$ nanoparticles. Table 7.2 is an example for the shelling injections using the calculated amounts from Table 6.6.

Table 7.2. Example Shelling Amounts and Timetables

\begin{tabular}{|c|c|c|}
\hline Shell & Injection Amount & Injection Time \\
\hline $\mathrm{Zn}$ & $1.20 \mathrm{~mL} \mathrm{Zn}$ & $2: 47 \mathrm{pm}$ \\
\hline $\mathrm{ZnS}$ & $1.20 \mathrm{~mL} \mathrm{~S}$ & $3: 05 \mathrm{pm}$ \\
\hline $\mathrm{ZnS}+\mathrm{Zn}$ & $1.64 \mathrm{~mL} \mathrm{Zn}$ & $3.23 \mathrm{pm}$ \\
\hline $\mathrm{ZnS}+\mathrm{ZnS}$ & $1.64 \mathrm{~mL} \mathrm{~S}$ & $3: 41 \mathrm{pm}$ \\
\hline $\mathrm{ZnS}+\mathrm{ZnS}+\mathrm{Zn}$ & $2.16 \mathrm{~mL} \mathrm{Zn}$ & $3: 57 \mathrm{pm}$ \\
\hline $\mathrm{ZnS}+\mathrm{ZnS}+\mathrm{ZnS}$ & $2.16 \mathrm{~mL} \mathrm{~S}$ & $4: 10 \mathrm{pm}$ \\
\hline
\end{tabular}




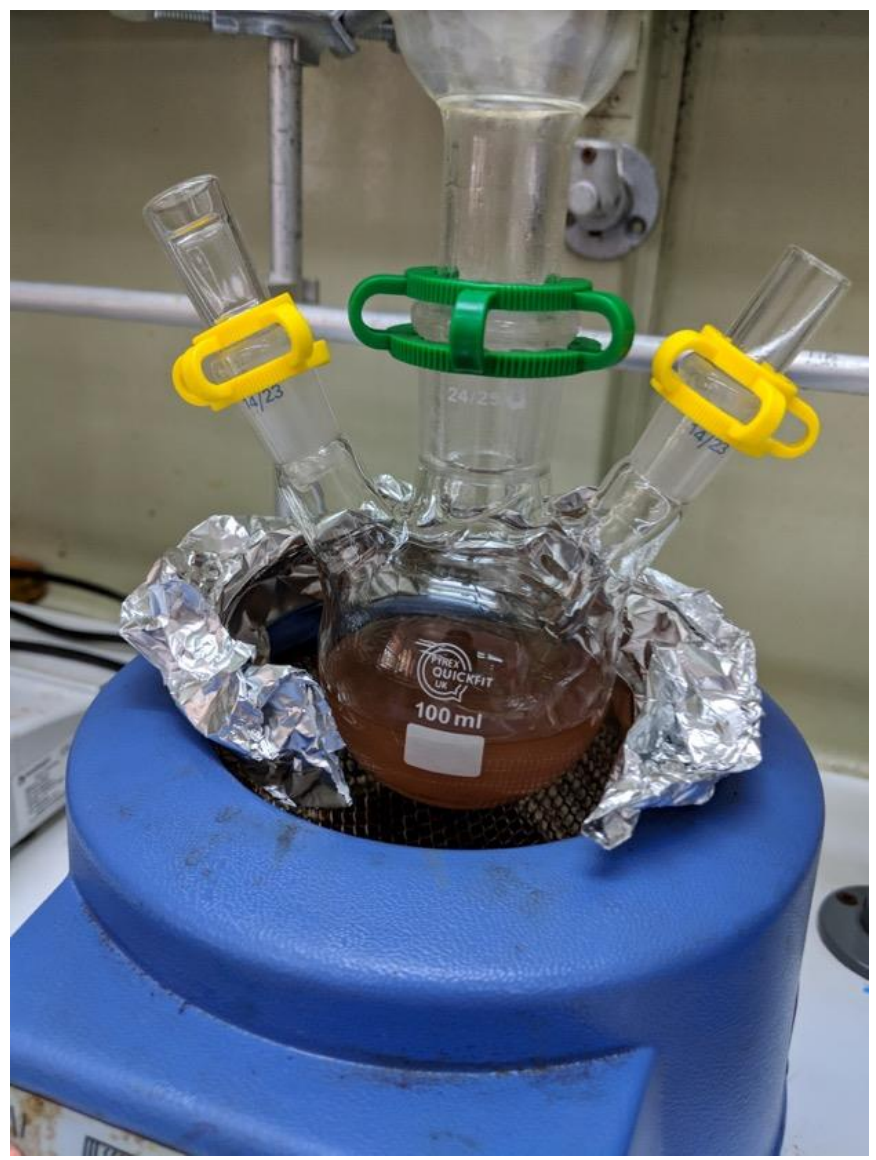

Figure 7.7. The finished product with the core-shell CdSe/ZnS quantum dots.

\subsection{Centrifugation of CdSe/ZnS Core-shell Quantum Dots}

The $\mathrm{CdSe} / \mathrm{ZnS}$ raw mixture is then centrifuged again under the same procedure and parameters used to purify and centrifuge the CdSe cores.

Similar to when the cores were synthesized, the next step is to centrifuge the raw mixture such that the coreshell CdSe/ZnS quantum dots can be transferred to a pure solvent system such as chloroform. Figure 7.8 below was the precipitate pattern after the first centrifuge cycle. Figure 7.9 was after it is the first discarded supernatant - the strong yellow color being indicative of the sulfur component and other contaminants such as octadecene. 


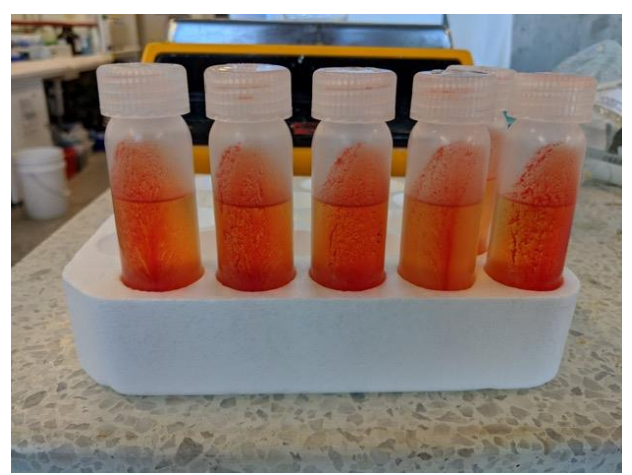

Figure 7.8. The precipitate pattern for the coreshell CdSe/ZnS quantum dots after the first centrifuge run is scattered and discontinuous.

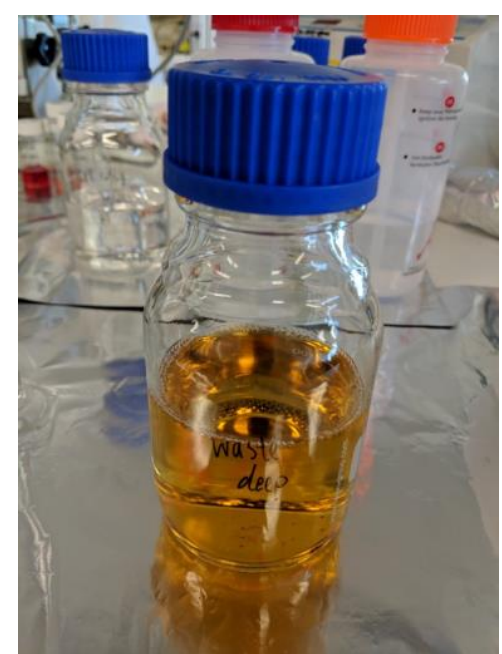

Figure 7.9. The first supernatant is very yellow compared to washing out the cores alone.

The second centrifuge step was unique in that in preparing for it when adding the purification mixtures, the nanoparticles easily separated out of the mixture and began to form the precipitate at the bottom of the mixture. This was expected since the shelling around the cores stabilize the structure and promote it from being immiscible in the solvents added within the purification mixtures. An example of this clear and distinct phase separation is provided in Figure 7.10 and Figure 7.11 for two of the centrifuge tubes. 


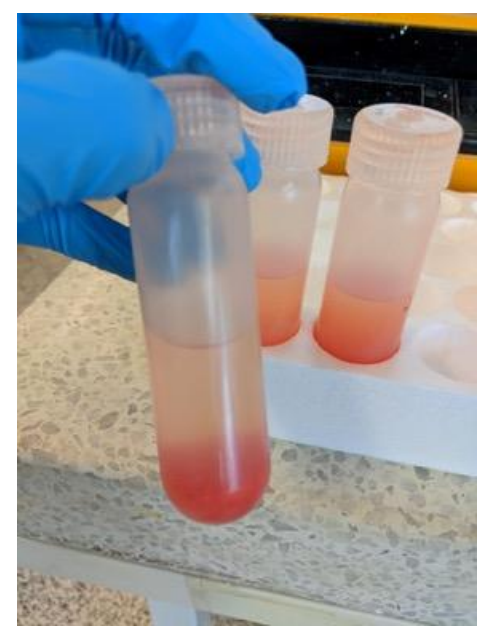

Figure 7.10. The quantum dots readily separated from the solvents even before centrifuging the sample.

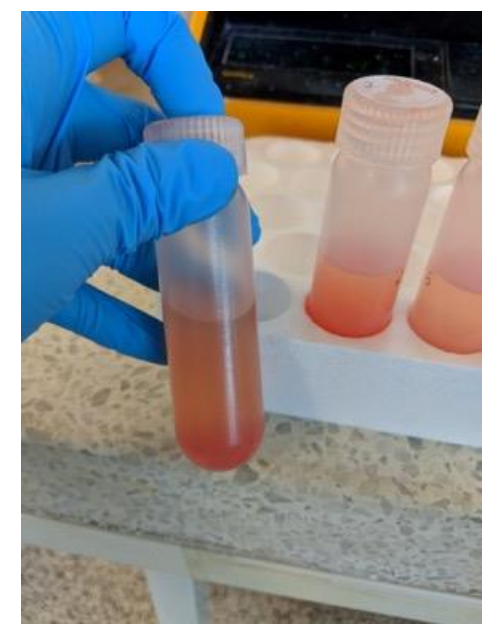

Figure 7.11. The same phenomena were exhibited with other centrifuge tubes as well.

The final precipitate containing the now shelled CdSe/ZnS quantum dots was then redissolved in $5 \mathrm{~mL}$ chloroform added using a calibrated pipet. Figures 7.12 and 7.13 below show the now purified core-shell quantum dots transferred over to a scintillation vial, and the latter fluorescence under a $395 \mathrm{~nm}$ UV source. 


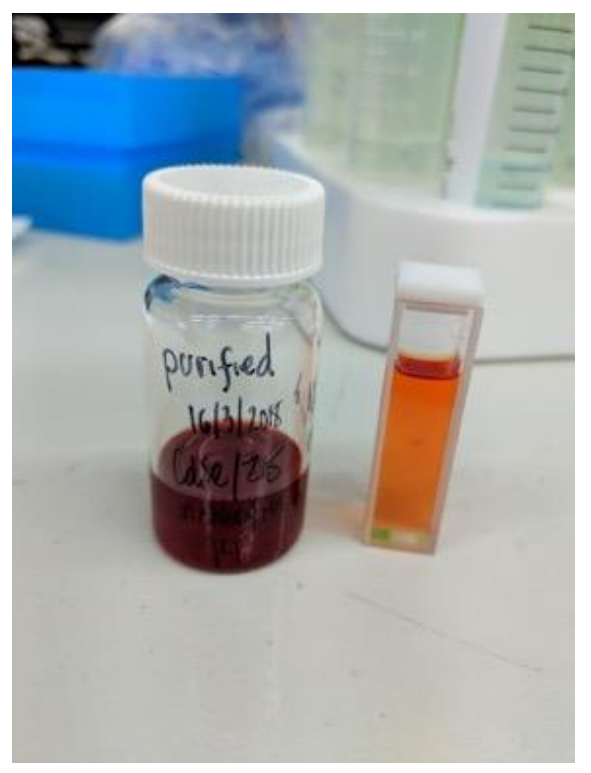

Figure 7.12. The CdSe/ZnS after purification deposited into chloroform before optical characterization.

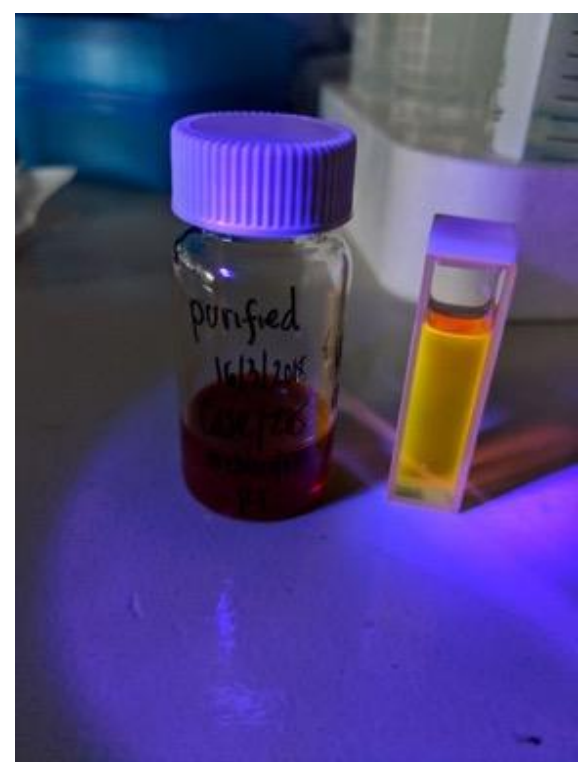

Figure 7.13. A bright orange fluorescence for the CdSe/ZnS under a $395 \mathrm{~nm}$ UV source.

\subsection{Optical Characterization Results for the CdSe/ZnS Core-Shell Quantum Dots}

The results of the optical characterization results are shown below. Figure 7.14 is that of the cadmium selenide cores before shelling, while Figure 7.15 is the core-shelled CdSe/ZnS. It is expected that the wavelength should red shift because the nanoparticle size increases due to the $\mathrm{ZnS}$ shelling outside the CdSe cores. 


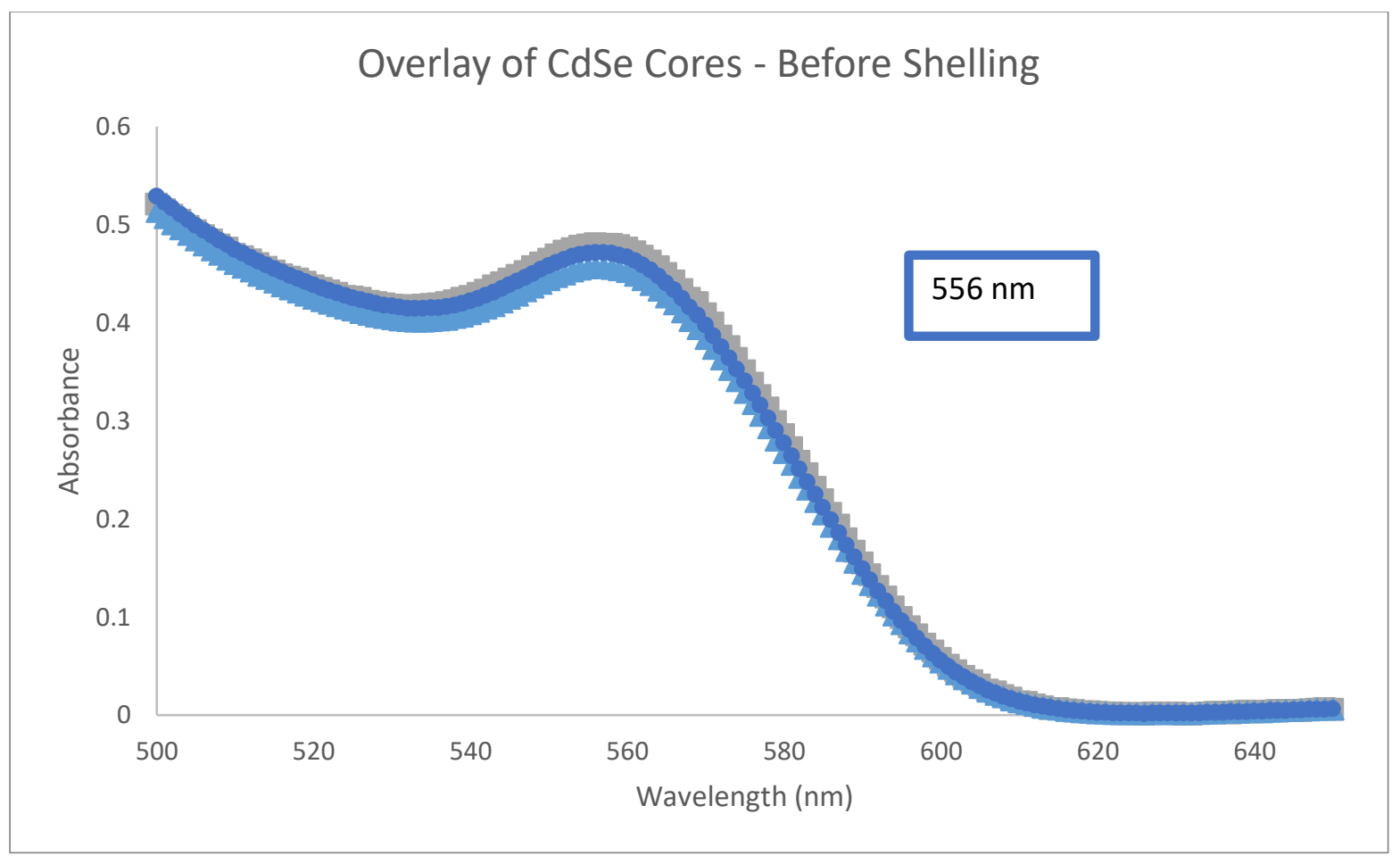

Figure 7.14. UV-Vis measurements for the CdSe cores before shelling.

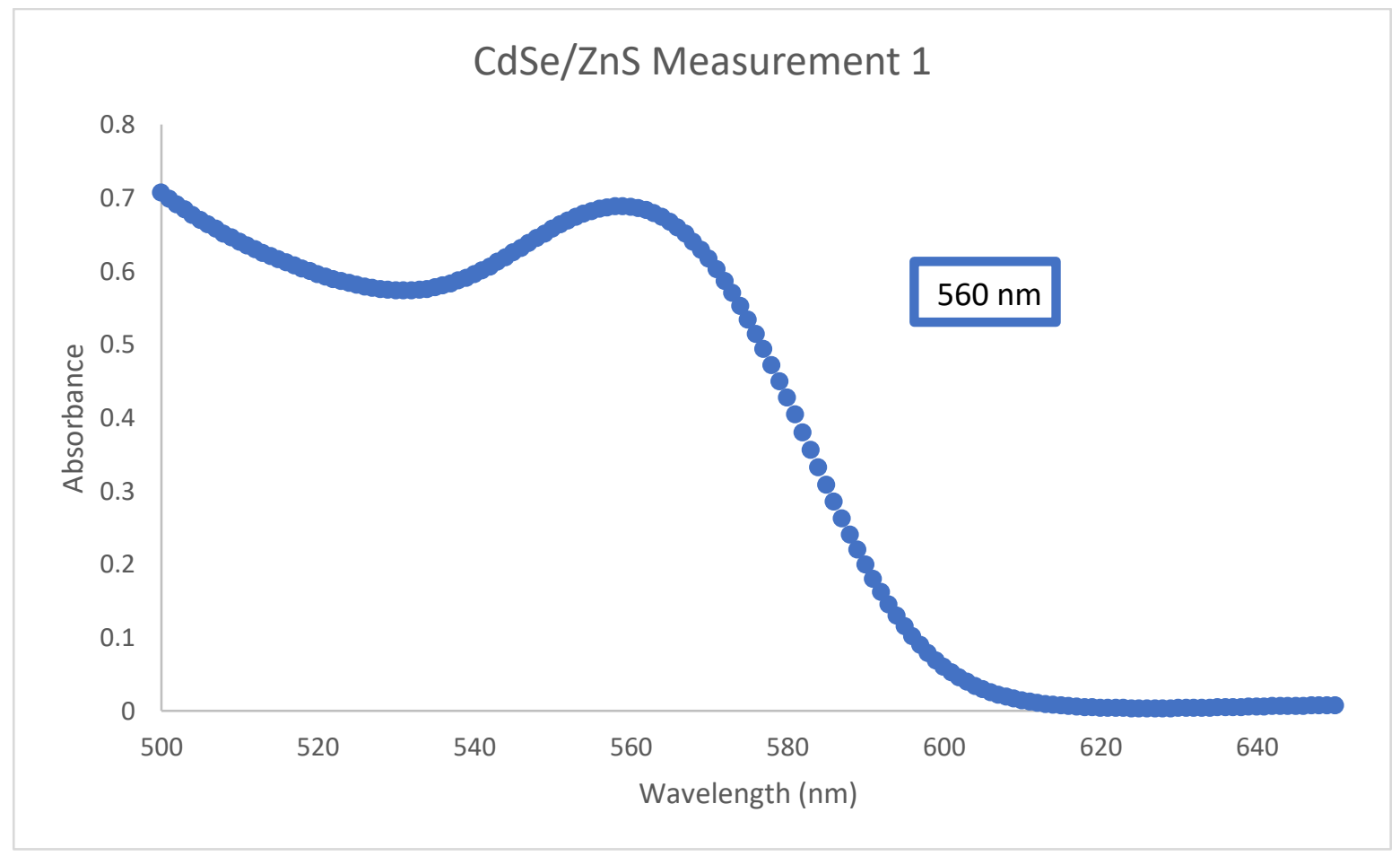

Figure 7.15. UV-Vis measurements for the CdSe/ZnS core-shell quantum dots after shelling. 
An overlay of the UV-Vis measurements before and after shelling for a different system is shown in Figure 7.16. In this overlay, it is observed that post-shelling the absorbance and intensity of the peak is much greater than before. Even though these are just arbitrary units, this suggests that the shelled cores are optically performing better than before when the system was just quantum dot cores. Ideally, the UV-Vis measurements before and after shelling should result not only in a slight red shift towards a larger wavelength, but the peak intensity should also be increased due to the nanoparticle sandwiching effect.

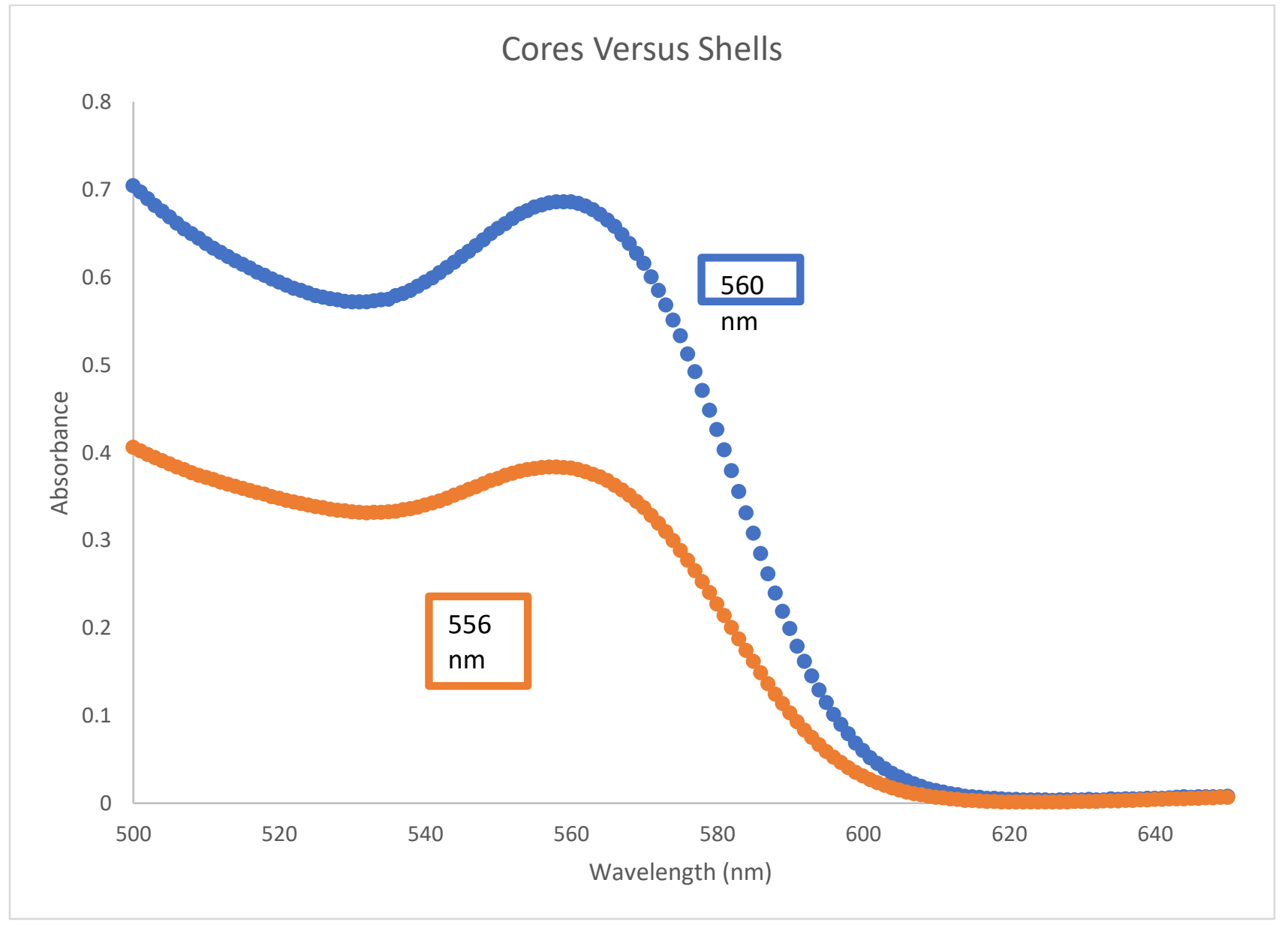

Figure 7.16. UV-Vis measurements comparing before and after shelling - a red shift is expected since shelling increases nanoparticle size. 
The UV-Vis results are also reflected in the fluorescence results, where a similar red shift is observed due to the overall particle size increasing. Measurements were taken before shelling in Figure 7.17 and after shelling in Figure 7.18.

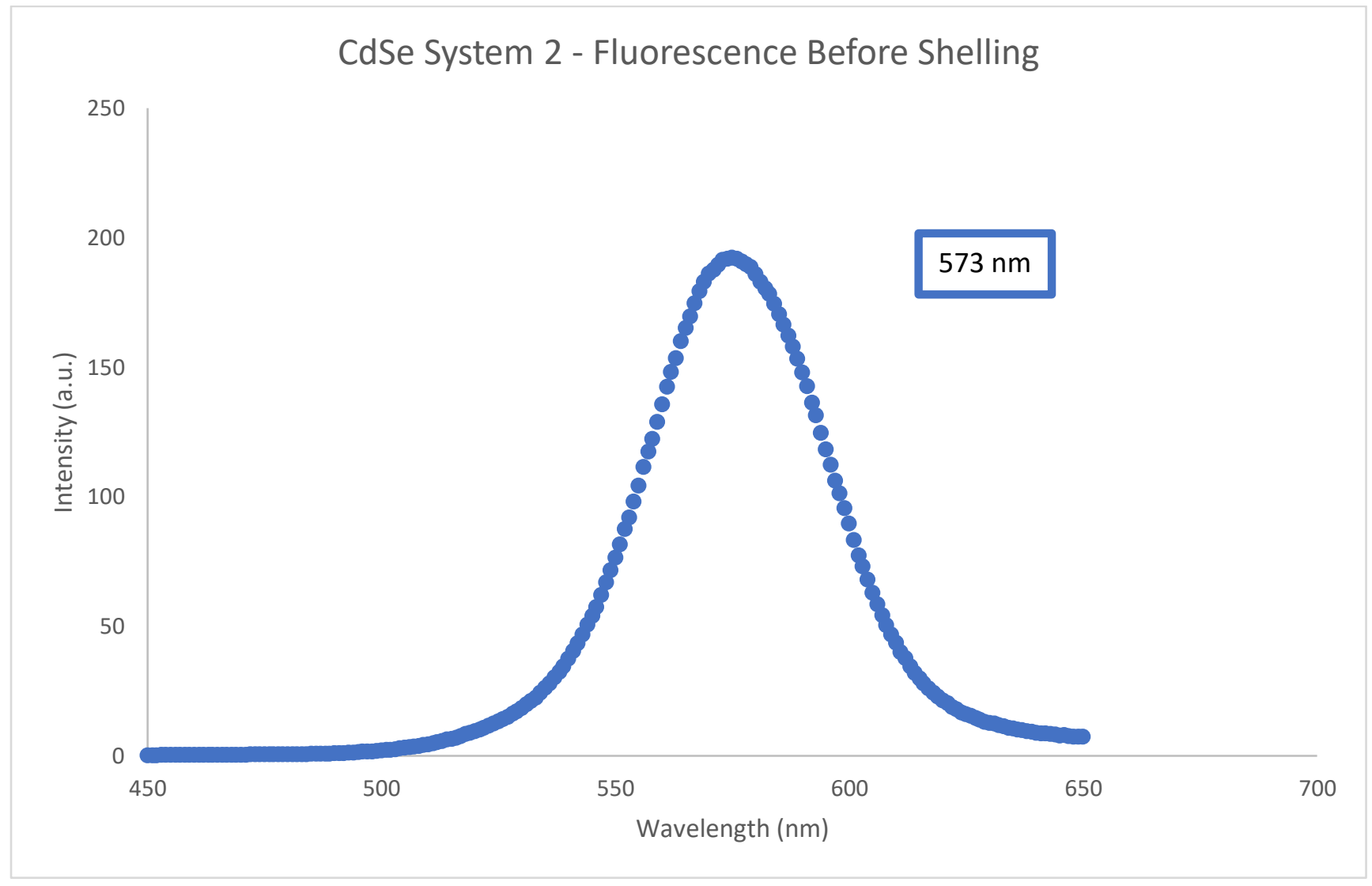

Figure 7.17. Fluorescence measurement for the CdSe cores only before shelling. 


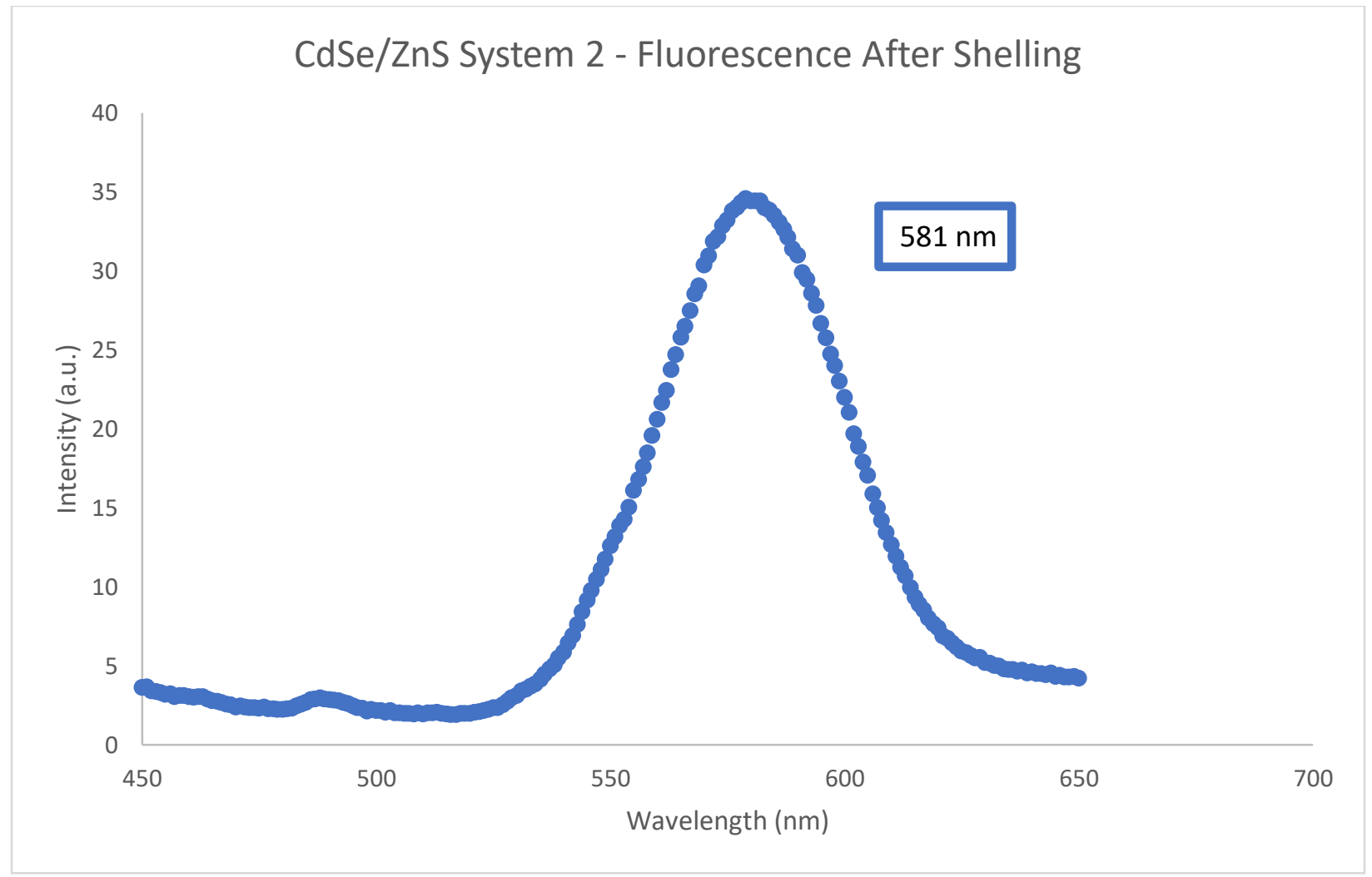

Figure 7.18. Fluorescence measurement for the CdSe/ZnS core-shell quantum dots after shelling.

Despite the shift in the wavelength demonstrating that the shelling procedure was successful, one major detail that is a deterrent to the procedure is the difference in intensity. There are several factors that may have resulted in the arbitrary unit being measured differently. However, even when normalizing the data, it is noteworthy to mention that the fluorescence intensity is much lower after shelling compared to before. An overlay of the two above fluorescence measurements is provided in Figure 7.19, where this is easily seen. 


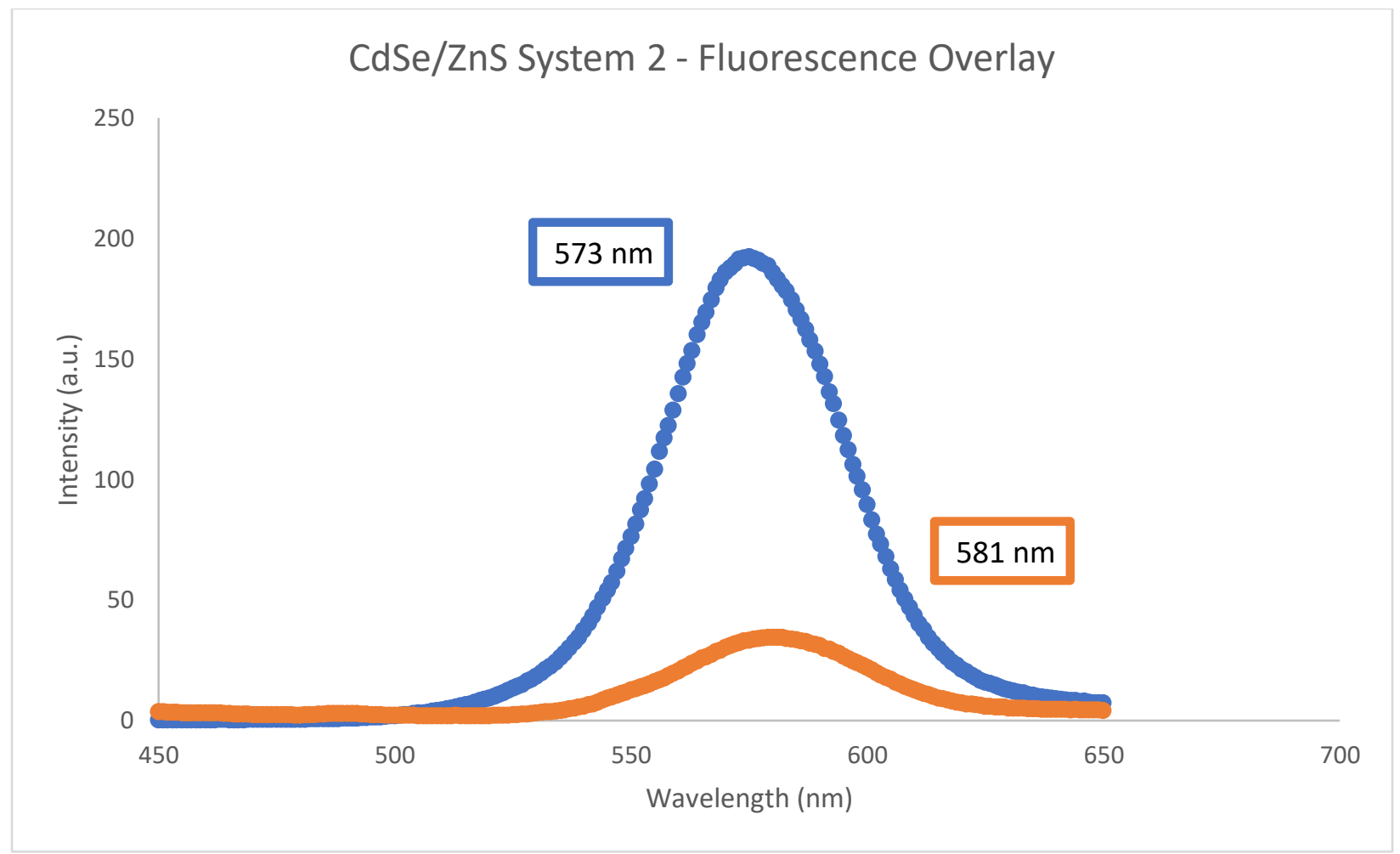

Figure 7.19. Fluorescence measurement overlay comparing before and after shelling - a red shift is expected since shelling increases nanoparticle size.

The fluorescence overlay presents an interesting question in how successful the shelling of the quantum dot cores was. Since the intensity decreased - despite it being an arbitrary unit - the expected nanoparticle sandwich did not work. The expected result after shelling the cores is for the intensity to be greater since the shell has a greater band gap energy compared to the core. There is also a narrower bandwidth. This may suggest that there was passivation of the cores, and that the additional purification and centrifugation in the shelling step helped further purify the system, resulting in increased performance. The goal here is to have a peak post-shelling that has a narrower bandwidth and thus a greater full width half maximum - in this case, only one is observed, suggesting that the shelling reaction could be improved for future experiments. Both overlays for the UV-Vis and fluorescence measurements are a great barometer towards seeing how successful the shelling procedure was performed in preparing the core-shell quantum dots. The key is to 
maximize the full-width-half-maximum of the peak, which can be accomplished by the peak becoming narrower and the peak becoming taller. 


\section{$\underline{8 \text { Integration into Coatings Systems }}$}

With the core-shell quantum dot systems synthesized and characterized, the next step is to integrate them into coatings before setting up an experiment to determine how they respond with corrosion and other coating aging mechanisms. A series of polymers and commercial paints of unknown composition were used as pilot studies where $100 \mu \mathrm{L}$ to $1 \mathrm{~mL}$ of core-shell quantum dot mixture in chloroform was deposited. After deposition, the mixture was mixed thoroughly for several minutes or with a Thinky mixer for the commercial paints and polymers. A list of the coatings used is summarized in Table 8.1.

The resulting mixed coatings containing quantum dots were applied onto three different types of substrates cut into approximately 1 inch by 1 inch dimensions. Glass substrates were cut from borosilicate microscope glass slides and cleaned with acetone before deposition. 99.9\% elemental copper plates and Al-2024-T3 were obtained and cut into the same dimensions. Both samples were abraded using a Scotchbrite sponge and cleaned with acetone afterwards.

Table 8.1. Summary of Polymers and Coatings Systems Used

\begin{tabular}{|c|c|c|}
\hline Number & Coating Name & Preparation Notes \\
\hline $1^{[39]}$ & Poly(vinyl chloride) - $80000 \mathrm{Mn}$ & $\begin{array}{l}0.17 \mathrm{~g} \text { PVC dissolved in } 6 \mathrm{~mL} \text { THF } \\
\text { and added } 0.34 \mathrm{~g} \text { 2-Nitrophenyl } \\
\text { octyl ether as plasticizer }\end{array}$ \\
\hline $2^{[40]}$ & $\begin{array}{l}\text { Poly(methyl methacrylate) }-12000 \\
\qquad M_{n}\end{array}$ & $\begin{array}{l}0.17 \mathrm{~g} \text { PMMA dissolved in } 6 \mathrm{~mL} \\
\text { chloroform }\end{array}$ \\
\hline $3^{[41]}$ & $\begin{array}{l}\text { Poly(methyl methacrylate) - } \\
120000 \mathrm{M}_{\mathrm{n}}\end{array}$ & $\begin{array}{c}0.17 \mathrm{~g} \text { PMMA dissolved in } 6 \mathrm{~mL} \\
\text { chloroform }\end{array}$ \\
\hline $4^{[42]}$ & $\begin{array}{l}\text { Rust inhibiting topcoat - Epoxy } \\
\text { enamel }\end{array}$ & $\begin{array}{l}\text { Stirred vigorously for } 10 \text { minutes } \\
\text { and placed in Thinky mixer for } 15 \\
\text { minutes }\end{array}$ \\
\hline
\end{tabular}




\begin{tabular}{|c|c|c|}
\hline $5^{[43]}$ & Feast \& Watson 2K Polyurethane & $\begin{array}{c}\text { 1:1 mixing between Parts A and B } \\
\text { - coating allowed to cure for } 5 \\
\text { days; used Thinky mixer }\end{array}$ \\
\hline $6^{[44]}$ & AkzoNobel High Solids 2K Epoxy & $\begin{array}{c}\text { 1:1 mixing between Parts } \mathrm{A} \text { and } \mathrm{B} \\
\text { — coating allowed to cure for } 5 \\
\text { days; used Thinky mixer }\end{array}$ \\
\hline $7^{[45]}$ & PPG Aerospace 2K Polyurethane & $\begin{array}{c}\text { 1:1 mixing between Parts } \mathrm{A} \text { and } \mathrm{B} \\
\text { - coating allowed to cure for } 5 \\
\text { days; used Thinky mixer }\end{array}$ \\
\hline
\end{tabular}

Images of the commercial and industrial paints are provided in Figures 8.1-8.4 below. 


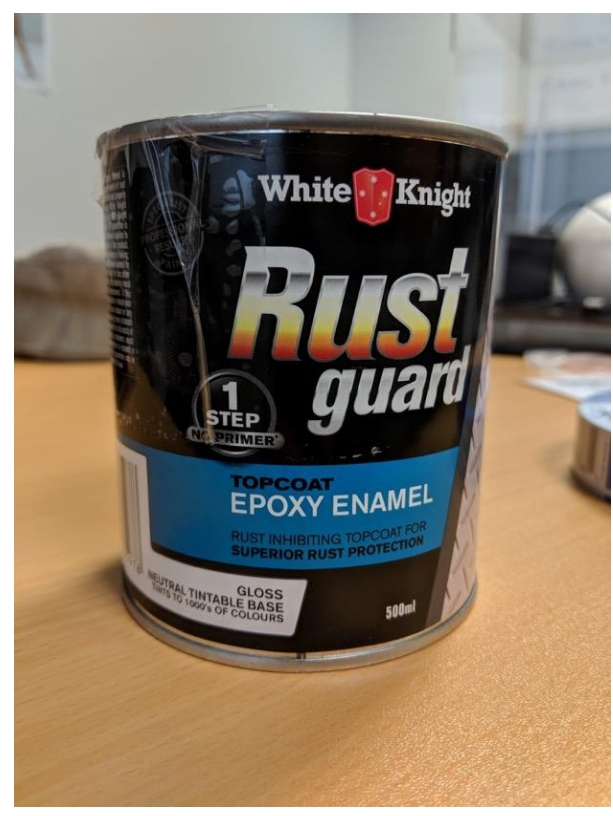

Figure 8.1. An image of the commercial rust inhibiting topcoat - epoxy enamel.

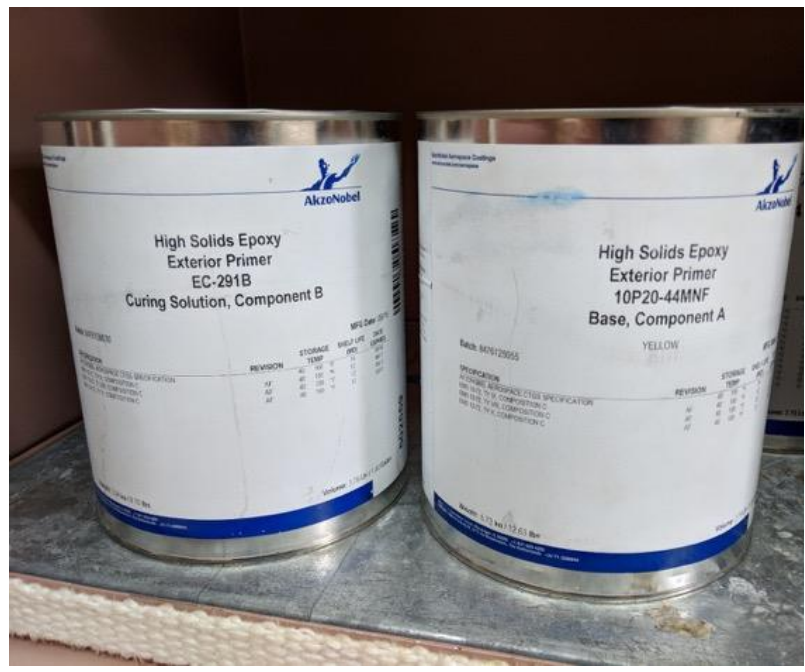

Figure 8.3. An image of the industrial use AkzoNobel

high solids $2 K$ epoxy.

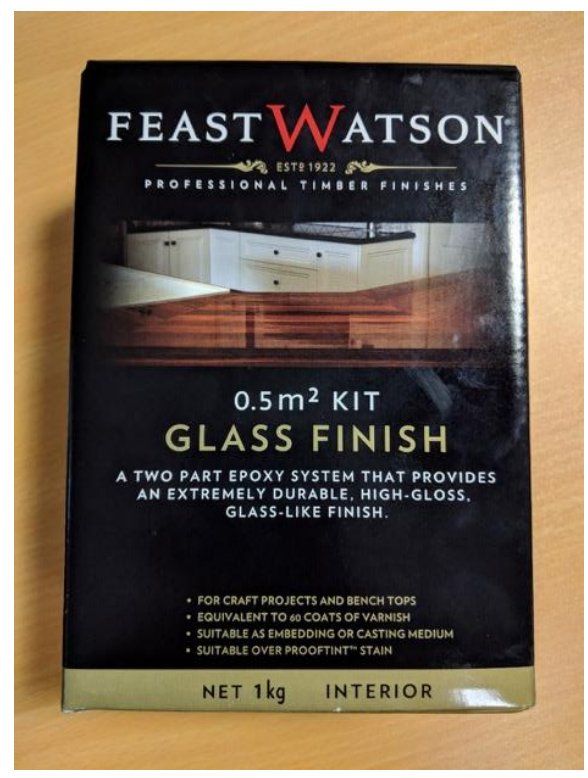

Figure 8.2. An image of the commercial Feast \& Watson 2 K polyurethane.

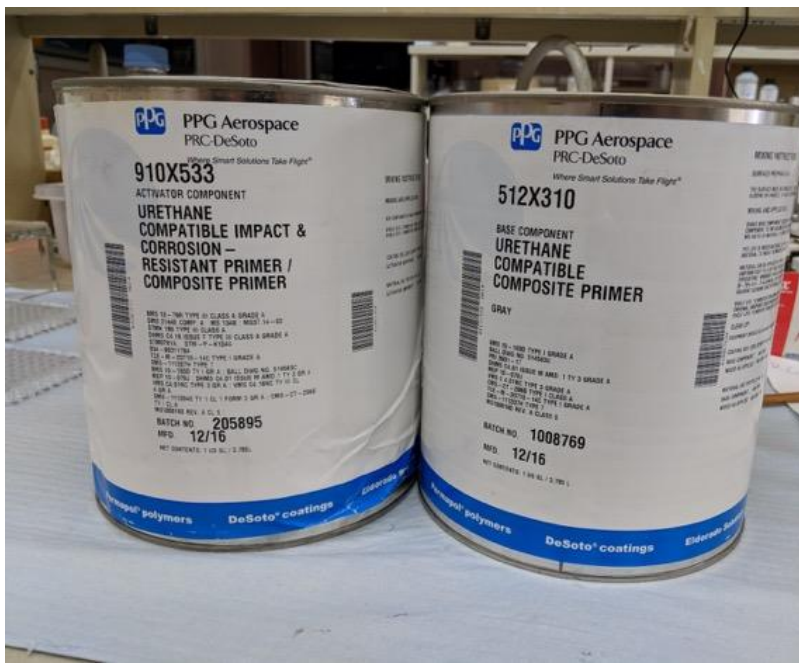

Figure 8.4. An image of the industrial use $P P G$

Aerospace $2 \mathrm{~K}$ polyurethane. 


\section{$\underline{\text { 8.1 Deposition of Quantum Dots into Coatings }}$}

All coatings were deposited onto their respective substrates in triplicate. The following figures demonstrate what these coatings look like atop the substrates for the different systems. Table 8.2 is for the polymers systems, while Table 8.3 is for the commercial paints coatings systems.

Table 8.2. Deposition into Polymers

PMC


Table 8.3. Deposition into Commercial and Specialty Paint Systems

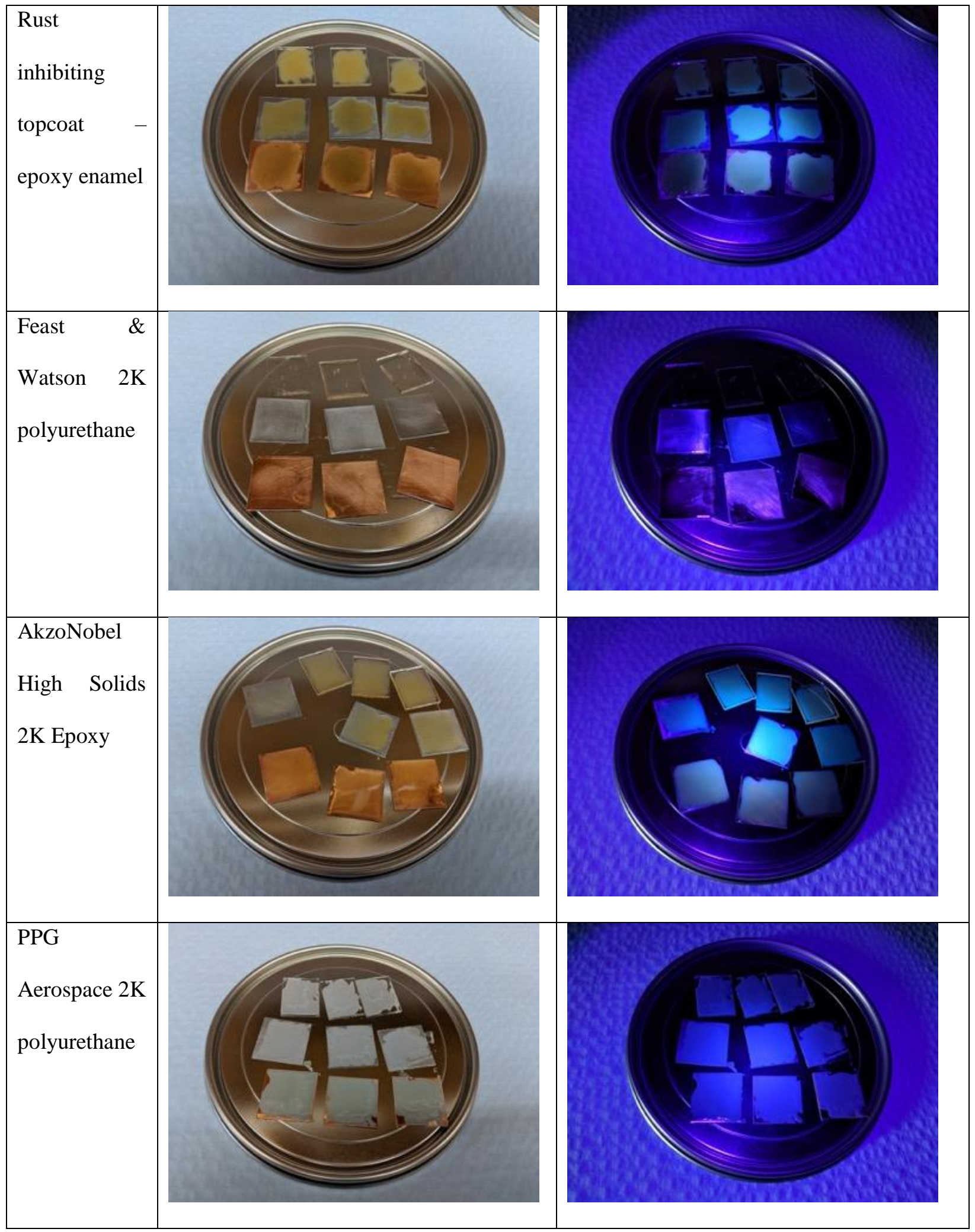


The fluorescence that is expected from the quantum dots is not readily detected in the coatings after deposition and integration into them. This may be due to several reasons, the first being that the quantum dots may have reacted upon mixing with the coating. This can be solved by treating the quantum dots with different capping agents that modify the reactivity of the surface such that it will not interact with the components in the coating. One possible direction is to obtain broad wavelength fluorescence measurements to determine if the photoluminescence detected by the quantum dots are still present after deposition. Even if the peaks are considerably smaller than the oversaturated peaks from the coating, they will still be detectable, and this peak can be used as a fingerprint to describe what happened to the quantum dots during the mixing stage. This will allow for a clear conclusion as to whether the quantum dots are still fluorescent after mixing with the coating.

Ultimately, this data requires more careful investigation towards the stability of the quantum dots and their response upon interacting with the polymer and coatings systems. If a reaction occurred upon mixing, a new strategy must be considered to passivate the surface.

\section{$\underline{\text { 8.2 Preliminary Immersion Aging Testing }}$}

After coating the samples, despite the fluorescence not being strong enough to overcome the oversaturation from the solvent systems, preliminary immersion testing was performed. All samples were placed into small beakers filled with $5 \% \mathrm{NaCl}$ mixture and allowed to age under immersion for 3 days. An example of the benchtop setup for the Feast \& Watson 2K polyurethane is shown in Figure 8.5. 


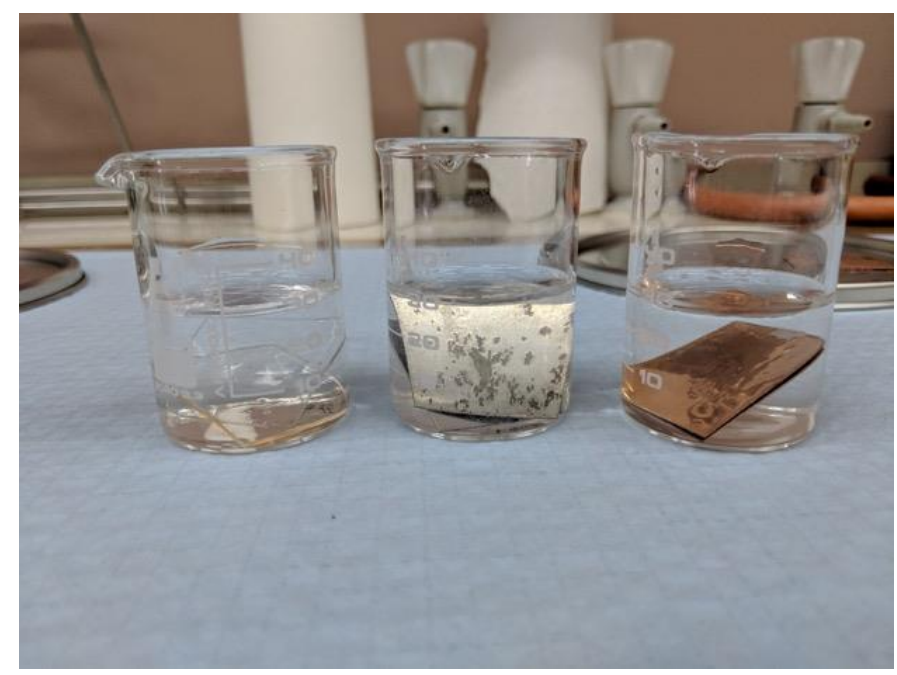

Figure 8.5. All the substrates and samples were immersed in 5\% $\mathrm{NaCl}$ mixture for at least 3 days.

The following tables show the aftermath of removing the coated substrates from the immersion soak testing. Here some of the samples exhibit some surface damage due to the salinity and water in the mixture, however, fluorescence is not observed under a $395 \mathrm{~nm}$ UV fsource. This means that the saturation issue is still masking the quantum dot fluorescence or perhaps the quantum dots did not survive long enough and are no longer active once transferred to the coating system. Table 8.4 has images of the polymers systems, while Table 8.5 has the commercial paint coatings - both exhibit the same issues with no fluorescence under a $395 \mathrm{~nm}$ UV source.

Table 8.4. Post-Immersion for Polymers

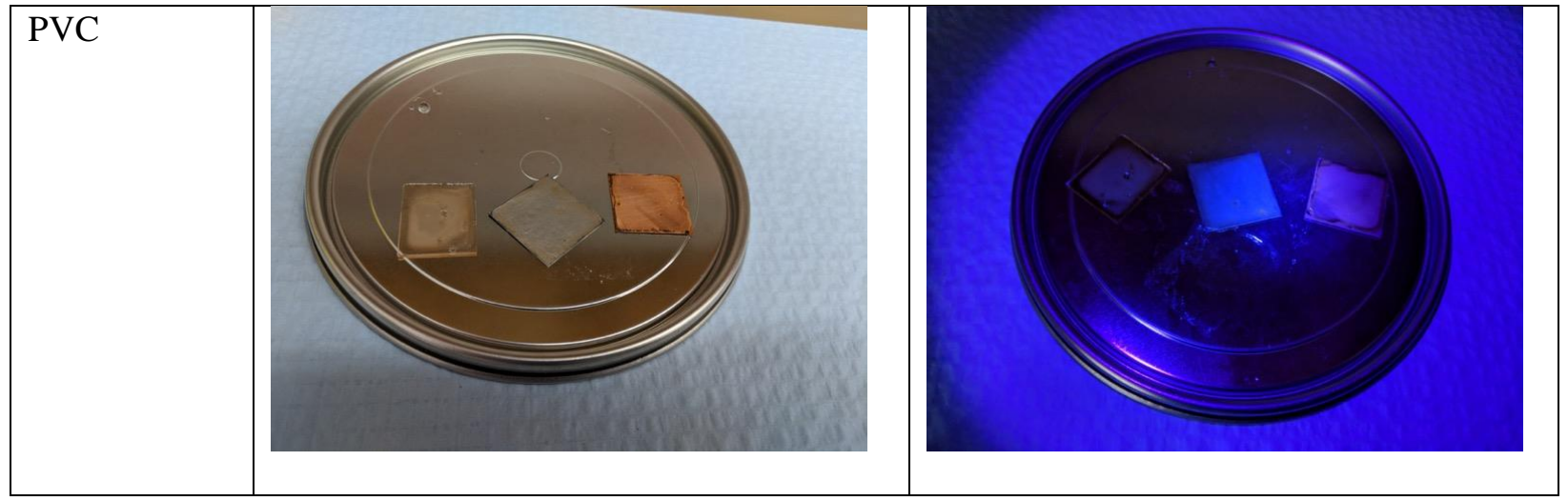




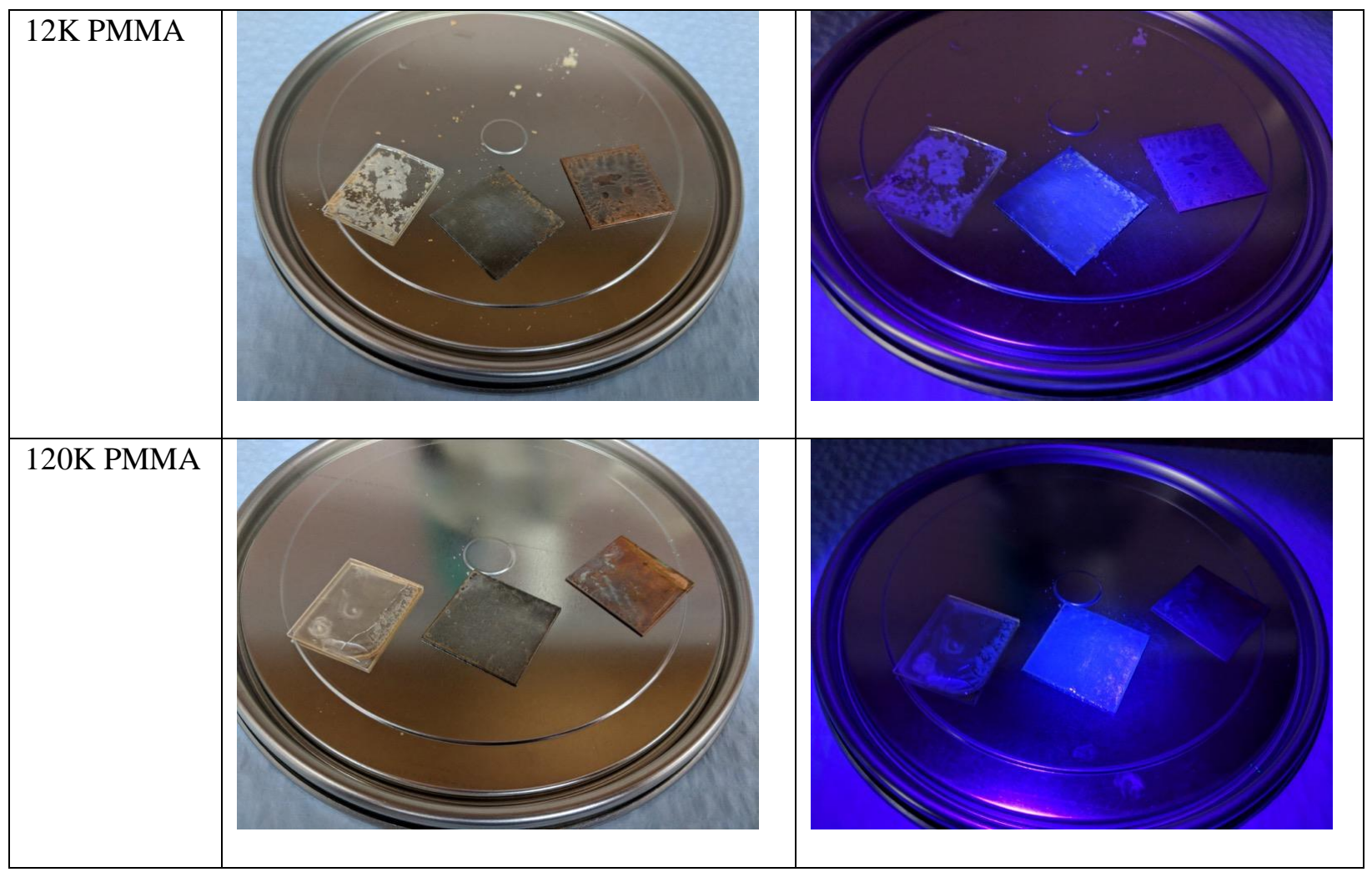

Table 8.5. Post-Immersion for Commercial and Specialty Paint Systems

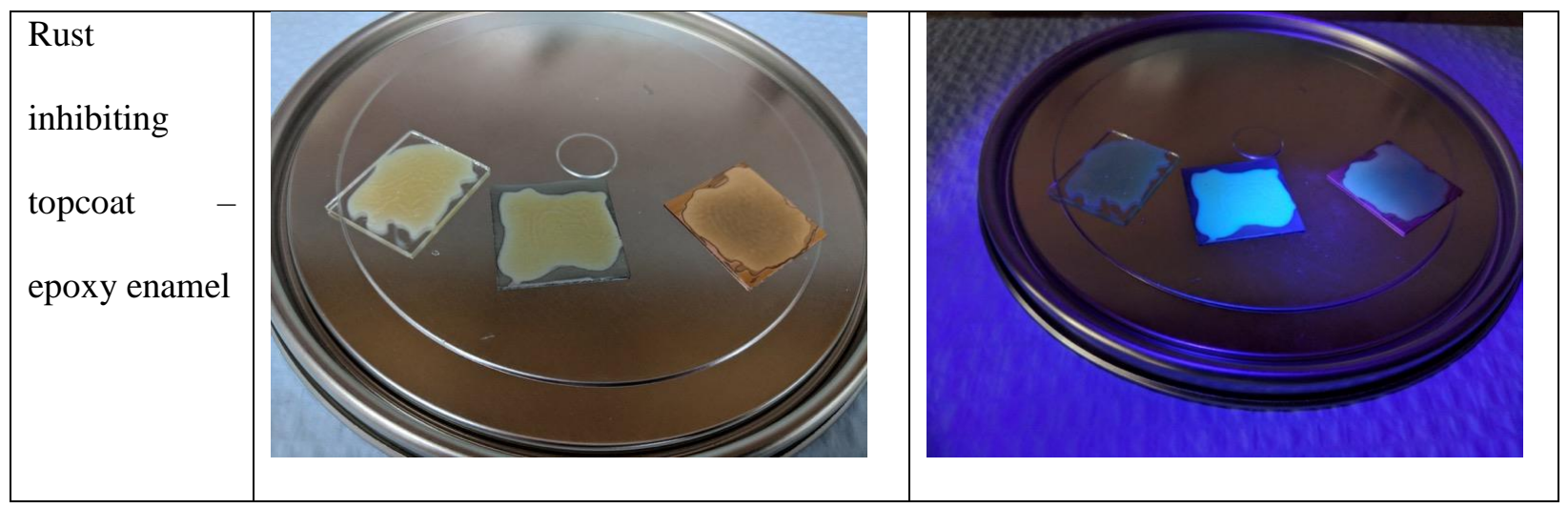




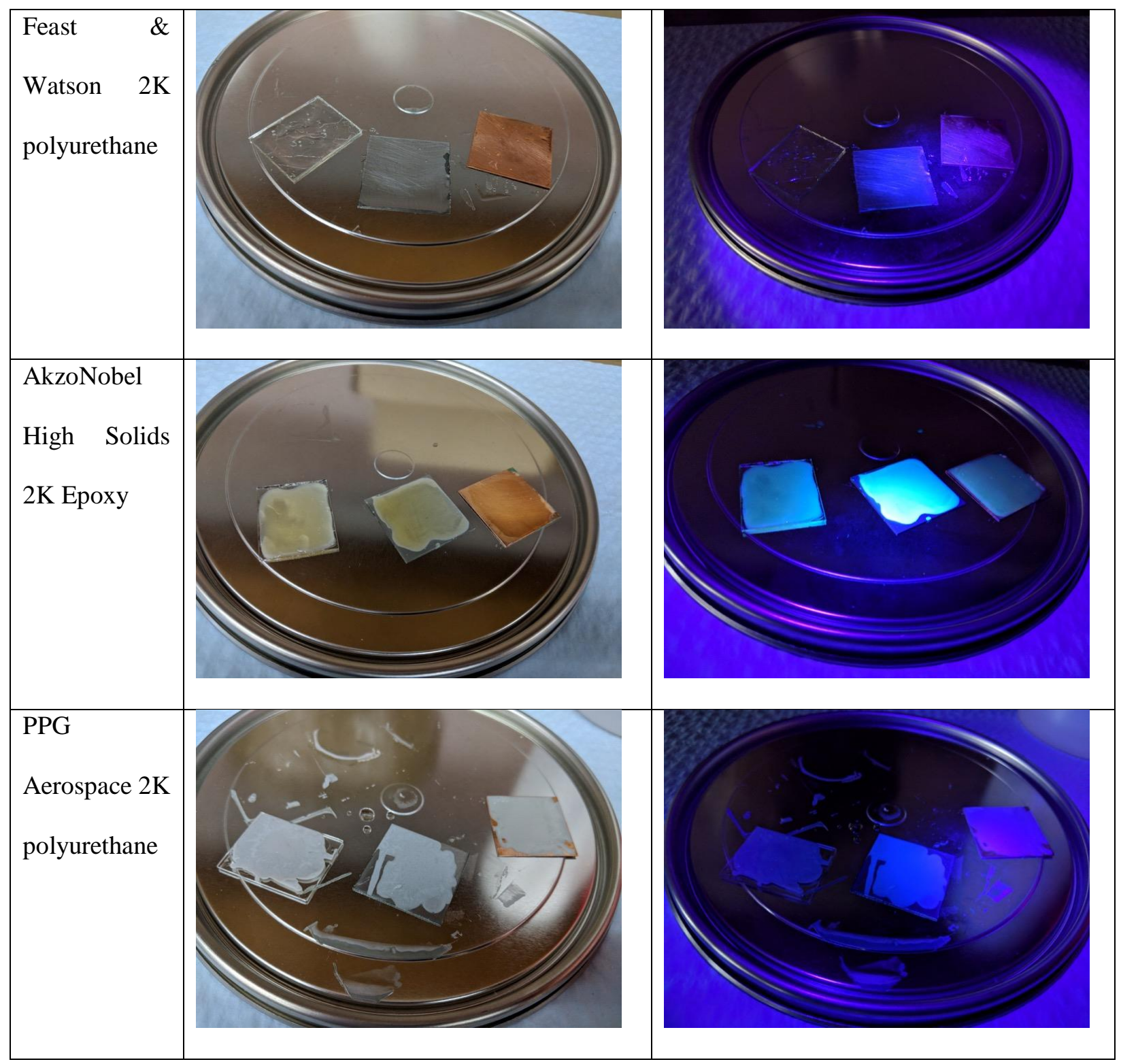




\section{Moving Forward and Future Directions}

From this research, core-shell quantum dots were successfully synthesized and researched. The physics behind their construction and how they operate and result in their characteristic fluorescence was explored, and this knowledge was expanded to successfully shelling the quantum dot cores through assumptions based on their bond lengths and particle sizes. Despite this, there is still room for improvement in modeling the nanoparticle size and shape to build better models for predicting and estimating the amounts needed to fully coat and shell the quantum dot cores. Optical characterization results through UV-Vis and fluorescence verify that the shelling of the cores was a successful reaction.

Future work should build upon the foundation that is set with the synthesis of core and core-shell quantum dot systems. There are a vast number of alternative syntheses that can be explored, however this research focuses on and masters that of $\mathrm{CdSe} / \mathrm{ZnS}$ core-shell quantum dots. Alternative synthesis techniques not explored in this research involved the use of phase-transfer to take the $\mathrm{CdSe} / \mathrm{ZnS}$ quantum dots from being soluble only in chloroform to being able to be used in water. This is a technique that definitely should be explored because it will widen the breadth of possible coatings that fluorescence quenching and thereby sensing of metal ions by quantum dots can be applicable to. The addition of the phase transfer and ligand exchange will allow for other systems to be integrated with quantum dots. For example, one ligand exchange can allow for the quantum dots to be dispersed in water and not just alcohol or chloroform systems. This will allow for integration of the quantum dots into systems where water is the primary carrier solvent such as waterborne paint coatings.

Additionally, other characterization methods can also be utilized to further and fully characterize the many properties of quantum dot materials. For example, particle size can alternatively be measured using dynamic light scattering, and SEM and other imaging techniques can be utilized to get a better picture of how these quantum dots appear at the nanoscale. Fluorescence mapping can be utilized to determine if there are small fluorescence peaks that correlate to the quantum dot systems. Investigating the change in that peak before 
and after integration into coatings can provide an additional perspective as to how the system is quenched and answer whether the system is disrupted or oversaturated by the coating. 


\section{$\underline{\text { References }}$}

[1] Vasudevan, D., Gaddam, R.R., Trinchi, A., Cole, I. Core-shell quantum dots: Properties and applications. Journal of Alloy and Compounds, 2015, Vol. 636, pages 395-404.

[2] Fernando, R.F. Sung, L.P. Nanocomposite and Nanostructured Coatings: Recent Advancements. Nanotechnology Applications in Coatings, ACS Symposium Series, 2009, doi: 10.1021/bk-20091008.ch001

[3] ASTM E2456-02. Standard Terminology Relating to Nanotechnology, ASTM International, West Conshohocken, PA, 2012, www.astm.org.

[4] Scientific Committee on Emerging and Newly Identified Health Risks. Scientific Basis for the Definition of the Term "Nanomaterial." European Commission, Directorate General for Health and Consumers, 2010, doi: 10.2772 .

[5] Madbouly, S.A., Otaigbe, J.U., Nanda, A.K., Wicks, D.A. Polyurethane-Polyhedral Oligomeric Silsesquioxanes Nanostructured Hybrid Dispersions by the Prepolymer Mixing Process. ACS Symposium Series, American Chemical Society: Washington, DC, 2010.

[6] Gao, F., Gu, Z. Melting Temperature of Metallic Nanoparticles. Handbook of Nanoparticles, 2016, pages 661-690.

[7] Fryer, D.S., Nealey, P.F., de Pablo, J.J. Thermal Probe Measurements of the Glass Transition Temperature for Ultrathin Polymer Films as a Function of Thickness. Macromolecules, 2009, Vol. 33, pages 6439-6447.

[8] Jiang, Q., Lang, X.Y. Glass Transition of Low-Dimensional Polystyrene. Macromolecular Rapid Communications, 2004 Vol. 25, pages 825-828. 
[9] Clarke, M.F., Paiz, A.A., Wilson, C.L., Brickweg, L.J., Floryancic, B.R., Fernando, R.H. Effects of Alumina on Polyurethane Clear Coating Properties. Nanotechnology Applications in Coatings, ACS Symposium Series, 2009, doi: 10.1021/bk_2009_1008.ch011

[10] Nguyen, Q.T., Baird, D.G. Preparation of Polymer-Clay Nanocomposites and Their Properties. Advances in Polymer Technology, 2007, Vol. 25, No. 4, pages 270-285.

11] Tung, H., Thanh, D.H., Lam, V.Q. The CdS/CdSe/ZnS photoanode cosensitized solar cells based on Pt, CuS, Cu 2 S, and PbS counter. Advances in Optoelectronics, 2014, Vol. 12, pages 9-16.

[12] Vasudevan, D., Trinchi, A., Hardin, S.G., Cole, I.S. Fluorescent heavy metal cation sensing with water dispersible 2MPA capped CdSe/ZnS quantum dots. Journal of Luminescence, 2015, Vol. 166, pages $88-92$.

[13] Zhu, Y., Free, M.L., Woollam, R., Durnie, W. A review of surfactants as corrosion inhibitors and associated modeling. Progress in Materials Science, 2017, Vol. 90, pages 159-223.

[14] Groysman, A. Corrosion for Everybody. SpringerLink: New York, 2010.

[15] Lange, J., Wyser, Y. Recent Innovations in Barrier Technologies for Plastic Packaging - a Review. Packing Technology and Science, 2003, Vol. 16, pages 149-158.

[16] Fateh, A., Aliofkhazraei, M., Rezvanian, A.R. Review of corrosive environments for copper and its corrosion inhibitors. Arabian Journal of Chemistry, 2017. http://dx.doi.org/10.106/j.arabjc.2017.05.021.

[17] Wicks, Z.W., Jones, F.N., Pappas, S.P., Wicks, D.A. Organic Coatings: Science and Technology, Third Edition. Wiley Publishing: New Jersey, 2007. 
[18] Lyon, S.B., Bingham, R., Mils, D.J. Advances in corrosion protection by organic coatings: What we know and what we would like to know. Progress in Organic Coatings, 2017, Vol. 102, pages 2-7.

[19] Cao, Y., Stavrinadis, A., Lasanta, T., So, D., Konstantatos, G. The role of surface passivation for efficient and photostable PbS quantum dot solar cells. Nature Energy, 2016, Vol. 1, page 1603516043.

[20] Alemu, Y.A., Albuquerque, G.H., Herman, G.S. Enhanced photoluminescence from $\mathrm{CuInS}_{2} / \mathrm{ZnS}$ quantum dots: Organic superacid passivation. Materials Letters, 2018, Vol. 219, pages 178-181.

[21] Zherebetskyy, D., Scheele, M., Zhang, Y., Bronstein, N., Thompson, C., Britt, D., Salmeron, M., Alivisatos, P. Wang, L.N. Hydroxylation of the surface of PbS nanocrystals passivated with oleic acid. Science, 2014, Vol. 334, pages 1380-1384.

[22] Moreels, I., Justo, Y., De Geyter, B., Haustraete, K., Martins, J.C., Hens, Z. Size-Tunable, Bright, and Stable PbS Quantum Dots: A Surface Chemistry Study. ACS Nano, 2011, Vol. 5, No. 3, pages 2004-2012.

[23] Gao, J., Johnson, J.C. Charge Trapping in bright and Dark States of Coupled PbS Quantum Dot Films. ACS Nano, 2012, Vol. 6, No. 4, pages 3292-3303.

[24] Nazeer, A.A., Madkour, M. Potential use of smart coatings for corrosion protection of metals and alloys: A review. Journal of Molecular Liquids, 2018, Vol. 253, pages 11-22.

[25] Sigma Aldrich. Cadmium oxide chemical properties from Sigma Aldrich documentation and specification sheets, 2018. Retrieved 6/11/2018 at https://www.sigmaaldrich.com/catalog/product/aldrich/202894?lang=en\&region=US. 
[26] Sigma Aldrich. Selenium chemical properties from Sigma Aldrich documentation and specification sheets, 2018. Retrieved 6/11/2018 at https://www.sigmaaldrich.com/catalog/product/aldrich/229865?lang=en\&region=US

[27] Sigma Aldrich. Zinc oxide chemical properties from Sigma Aldrich documentation and specification sheets, 2018. Retrieved 6/11/2018 at https://www.sigmaaldrich.com/catalog/product/sigald/96479?lang=en\&region=US

[28] Sigma Aldrich. Sulfur chemical properties from Sigma Aldrich documentation and specification sheets, 2018. Retrieved 6/11/2018 at https://www.sigmaaldrich.com/catalog/product/aldrich/213292?lang=en\&region=US

[29] Sigma Aldrich. Trioctylphosphine chemical properties from Sigma Aldrich documentation and specification sheets, 2018. Retrieved 6/11/2018 at https://www.sigmaaldrich.com/catalog/product/aldrich/117854?lang=en\&region=US

[30] Sigma Aldrich. Oleic acid chemical properties from Sigma Aldrich documentation and specification sheets, 2018. Retrieved 6/11/2018 at https://www.sigmaaldrich.com/catalog/product/aldrich/364525?lang=en\&region=US

[31] Sigma Aldrich. Octadecene chemical properties from Sigma Aldrich documentation and specification sheets, 2018. Retrieved 6/11/2018 at https://www.sigmaaldrich.com/catalog/product/sial/74738?lang=en\&region=US

[32] Sigma Aldrich. Oleylamine chemical properties from Sigma Aldrich documentation and specification sheets, 2018. Retrieved 6/11/2018 at https://www.sigmaaldrich.com/catalog/product/aldrich/o7805?lang=en\&region=US 
[33] Sigma Aldrich. Chloroform chemical properties from Sigma Aldrich documentation and specification sheets, 2018. Retrieved 6/11/2018 at https://www.sigmaaldrich.com/catalog/product/mm/cx1050?lang=en\&region=US

[34] Sigma Aldrich. Acetone chemical properties from Sigma Aldrich documentation and specification sheets, 2018. Retrieved 6/11/2018 at https://www.sigmaaldrich.com/catalog/product/mm/ax0115?lang=en\&region=US

[35] Sigma Aldrich. Ethanol chemical properties from Sigma Aldrich documentation and specification sheets, 2018. Retrieved 6/11/2018 at https://www.sigmaaldrich.com/catalog/product/sigald/459844?lang=en\&region=US

[36] Sigma Aldrich. Heptane chemical properties from Sigma Aldrich documentation and specification sheets, 2018. Retrieved 6/11/2018 at https://www.sigmaaldrich.com/catalog/product/sigald/34873?lang=en\&region=US

[37] Yu, W.W., Qu, L., Guo, W., Peng, X. Experimental determination of the Extinction Coefficient of CdTe, CdSe, and CdS Nanocrystals. Chemistry of Materials, 2003, Vol. 15, pages 2854-2860.

[38] Jasieniak, J., Smith, L., van Embden, J., Mulvaney, P. Re-examination of the Size-Dependent Absorption Properties of CdSe Quantum Dots. Journal of Physical Chemistry C, 2009, Vol. 113, pages $19468-19474$.

[39] Sigma Aldrich. Poly(vinyl chloride) chemical properties from Sigma Aldrich documentation and specification sheets, 2018. Retrieved 6/11 at https://www.sigmaaldrich.com/catalog/product/aldrich/189588?lang=en\&region=US 
[40] Sigma Aldrich. Poly(methyl methacrylate) $12000 M_{n}$ chemical properties from Sigma Aldrich documentation and specification sheets, 2018. Retrieved 6/11/2018 at https://www.sigmaaldrich.com/catalog/product/sial/81497?lang=en\&region=US

[41] Sigma Aldrich. Poly(methyl methacrylate) $120000 M_{n}$ chemical properties from Sigma Aldrich documentation and specification sheets, 2018. Retrieved 6/11/2018 at https://www.sigmaaldrich.com/catalog/product/sial/81503?lang=en\&region=US

[42] White Knight. Rust inhibiting topcoat chemical properties from White Knight documentation and specification sheets, 2018. Retrieved 6/11/2018 at https://www.whiteknightpaints.com.au/products/metal/white-knight-rust-guard/topcoat/whiteknight-rust-guard-epoxy-enamel/

[43] Feast \& Watson. Feast \& Watson 2 K polyurethane chemical properties from Feast \& Watson documentation and specification sheets, 2018. Retrieved 6/11/2018 at http://www.feastwatson.com.au/consumer/products/interior/product-details/1536

[44] PEXA Global. AkzoNobel High Solids 2 K epoxy chemical properties from AkzoNobel/PEXA documentation and specification sheets, 2018. Retrieved 6/11/2018 at http://www.pexa.com/shop/wp-content/uploads/2017/09/AkzoNobel-22-23-Series-2015.pdf

[45] PPG High Performance Coatings. PPG Aerospace 2 K polyurethane chemical properties from PPG Aerospace documentation and specification sheets, 2018. Retrieved 6/11/2018 at http://www.ppghighperformancecoatings.com/getmedia/0f13f761-e16b-4dc8-a9c51c0688daa842/TDS_95-8800.pdf.aspx?ext=.pdf 


\section{Appendix: Synthesis Procedure for CdSe/ZnS Quantum Dots}

\section{$\underline{\text { Synthesis of CdSe/ZnS Quantum Dots }}$}

\begin{tabular}{|l|l|l|l|}
\hline & Compound Name & Molar Mass $(\mathrm{g} / \mathrm{mol})$ & Density at $25^{\circ} \mathrm{C}(\mathrm{g} / \mathrm{mL})$ \\
\hline $\mathrm{CdO}$ & Cadmium Oxide & 128.41 & Powder \\
\hline $\mathrm{Se}$ & Selenium & 78.971 & Powder \\
\hline OA & Oleic acid & 282.47 & 0.895 \\
\hline TOP & Trioctylphosphine & 370.64 & 0.831 \\
\hline ODE & Octadecene & 252.49 & 0.789 \\
\hline ZnAce & Zinc acetate & 183.48 & Powder \\
\hline $\mathrm{S}$ & Sulfur & 32.065 & Powder \\
\hline
\end{tabular}

\section{Synthesis of CdSe Cores}

Preparation of Cd Precursor

1. Add $0.6 \mathrm{mmol} \mathrm{CdO}$, excess $3.4 \mathrm{~mL} \mathrm{OA}$, and $15 \mathrm{~mL} \mathrm{ODE}$ to a three-neck round bottom flask with stirring bar.

2. Heat to $110^{\circ} \mathrm{C}$ and evacuate under vacuum for $1 \mathrm{hr}$.

3. Switch to $\mathrm{N}_{2}$ flow and heat to $220^{\circ} \mathrm{C}$ for $1 \mathrm{hr}$ - the solution should clear up and become a pale yellow color.

4. Cool to $100^{\circ} \mathrm{C}$ and evacuate under vacuum for another $15 \mathrm{~min}$. DO NOT SWITCH VACUUM

\section{UNTIL COLD OR FLASK WILL BUMP}

5. Switch to $\mathrm{N}_{2}$ flow and heat to $220^{\circ} \mathrm{C}$ - leave this flask under $\mathrm{N} 2$ flow until injecting Se.

$\underline{\text { Preparation of Se Precursor }}$

1. Add $0.06 \mathrm{mmol}$ Se and $5 \mathrm{~mL}$ ODE to a three-neck round bottom flask with stirring bar. 
2. Heat to $110^{\circ} \mathrm{C}$ and evacuate under vacuum for $1 \mathrm{hr}$.

3. Switch to $\mathrm{N}_{2}$ flow and inject $0.6 \mathrm{~mL}$ TOP into the flask.

4. Then raise the temperature to $180^{\circ} \mathrm{C}$ and leave for $1 \mathrm{hr}$ - the powder should dissolve and the solution should appear optically clear.

Injecting the Se Precursor into the Cd Precursor

1. Rapidly inject the Se precursor into the Cd precursor - inject in one straight shot.

2. The Cd source should start to change color from the pale yellow color to a light red to a darker red. QUENCH THE SOLUTION IN A WATER BATH TO STOP THE QD NUCLEATION

3. The final color is indicative of the size of the QDs - if the solution turns black, the QDs will not work.

4. Allow the reaction to proceed for $90 \mathrm{~s}$ and quench in a water bath.

a. The time varies depending on the temperature of the cores and injection - best to go by your judgment and do it when it becomes a wine red color - remember, we want to avoid them going black or else the reaction fails.

\section{$\underline{\text { Centrifuging the CdSe Cores }}$}

1. Get some Falcon centrifuge tubes and pour out the bulk wine red solution equally into the tubes.

2. We need to use a specific mixture of solvents to "wash out" and separate the QDs from the dirty solvent it is currently in.

3. Add $10 \mathrm{~mL}$ chloroform, $12 \mathrm{~mL}$ acetone, and $25 \mathrm{~mL}$ ethanol to each Falcon tube and shake vigorously.

a. This uses A LOT of solvent so I typically scale down to $5 \mathrm{~mL}, 6 \mathrm{~mL}, 12.5 \mathrm{~mL}$.

4. Weigh each vial and balance before using in the centrifuge - add more ethanol to fill the missing weight.

5. Centrifuge at $17000 \mathrm{rpm}$ for $13 \mathrm{~min}$. 
6. Discard the supernatant - should be a yellow color from all the ODE and OA we used earlier.

7. Add the chloroform part of the magic mixture and shake vigorously to redissolve the QDs.

a. Then add the acetone and ethanol components.

8. Then centrifuge and repeat discarding the supernatant and repeat magic mixture one more time.

a. The second and third times the supernatant should be much clearer and less "yellow" than the first time.

9. Redissolve the final QDs power in $10 \mathrm{~mL}$ chloroform - use a calibrated pipet, this must be $10 \mathrm{~mL}$ exactly.

\section{$\underline{\text { Fluorescence and UV-vis Measurements }}$}

1. Run fluorescence measurements and UV-vis measurements on the purified CdSe cores.

2. Dilution factor is important $-\mathrm{I}$ do $100 \mu \mathrm{L}$ QDs solution into $3 \mathrm{~mL}$ chloroform.

a. Fluorescence - Excitation wavelength at $400 \mathrm{~nm}$, excitation and emission slit widths at 5 $\mathrm{nm}$, and scan range is $450-600 \mathrm{~nm}$.

b. UV-vis - Scan range over full spectrum.

\section{Determining Shell Ratios}

1. This part is important because it relies on the data from the UV-vis measurement.

a. Record the strongest absorption peak - record the wavelength and the absorbance intensity.

2. Input those two numbers into the shelling ratios spreadsheet.

a. $\quad 1^{\text {st }}$ absorption peak and Absorbance value (measured)

b. Add $25 \mathrm{~nm}$ and put this number into the higher range box

3. Adding those numbers will change the values in the last big table at the bottom - shelling ratios.

4. The orange boxes tell you what to add.

a. IE : $0.3350 \mathrm{~g}$ zinc acetate, $0.96 \mathrm{~mL} \mathrm{OA}, 1 \mathrm{~mL}$ OLM; then add $3 \mathrm{~mL}$ ODE to make final volume $5 \mathrm{~mL}$ 
b. For S : Add $0.0489 \mathrm{~g} \mathrm{~S}$ to $5 \mathrm{~mL}$ ODE

5. The injection volumes will be the numbers in the $\mathrm{mL}$ column.

a. IE, for the $\mathrm{Zn}$ shelling, $1.19 \mathrm{~mL}$ for the $1^{\text {st }}$ layer, $1.64 \mathrm{~mL} 2^{\text {nd }}$, and $2.17 \mathrm{~mL} 3^{\text {rd }}$.

6. The important numbers are the masses of the zinc/sulfur powder and what you need to add to make the injection precursors.

\section{Making the ZnS Shells around the CdSe Cores}

\section{Preparing the Bulk CdSe Cores for Shelling}

1. Add the bulk CdSe cores solution and $20 \mathrm{~mL}$ ODE to a three neck round bottom flask with stirring bar.

2. Heat slowly at $100^{\circ} \mathrm{C}$ with $\mathrm{N}_{2}$ flow and leave one of the necks open so the chloroform in the bulk solution escapes - assumption is that $\mathrm{N}_{2}$ flow will "push out" and help the chloroform escape the solution; leave for $1 \mathrm{hr}$.

3. Then switch to vacuum and evacuate under vacuum for $1 \mathrm{hr}$.

4. Switch back to $\mathrm{N}_{2}$ flow and increase the temperature slowly to $200^{\circ} \mathrm{C}$.

\section{Preparation of the $\mathrm{Zn}$ Shelling Injection Precursors}

1. Add ZnAce, OA, and $\mathrm{OE}$ to a three neck flask with a stirring bar.

2. Heat to $150^{\circ} \mathrm{C}$ and then add the OLM component - allow to mix for $30 \mathrm{~min}$ before continuing.

3. Cool to $110^{\circ} \mathrm{C}$ and evacuate under vacuum for $1 \mathrm{hr}$.

4. Then switch to $\mathrm{N}_{2}$ flow and heat to $260^{\circ} \mathrm{C}$ for $1 \mathrm{hr}$.

5. Cool back to $110^{\circ} \mathrm{C}$ and evacuate under vacuum for an additional $15 \mathrm{~min}$.

6. Switch back to $\mathrm{N}_{2}$ flow and leave at $180^{\circ} \mathrm{C}$.

\section{Preparation of the S Shelling Injection Precursors}

1. Add S and ODE to another three neck flask with a stirring bar. 
2. Heat to $110^{\circ} \mathrm{C}$ and evacuate under vacuum for $1 \mathrm{hr}$.

3. Then switch to $\mathrm{N}_{2}$ flow and heat to $180^{\circ} \mathrm{C}$ for $1 \mathrm{hr}$ - the solution should be clear and not cloudy.

\section{The Actual Injections for the Shells}

1. Use a syringe to draw up the needed volume from the spreadsheet.

2. Inject very slowly compared to the rapid injection for the cores - drip drip drip over a minute.

3. First inject the $\mathrm{Zn}$ into the cores and allow the reaction to run for $15 \mathrm{~min}$.

4. Then add the $\mathrm{S}$ volume next and $15 \mathrm{~min}$; then $\mathrm{Zn}$ and 15; etc. until 3 layers of $\mathrm{ZnS}$.

5. Allow to cool to RT and leave running overnight stirring.

6. Remove and centrifuge using the magic mixtures from before. 